\title{
LIGHT CURVES OF 213 TYPE Ia SUPERNOVAE FROM THE ESSENCE SURVEY
}

\author{
G. Narayan ${ }^{1,2,3}$, A. Rest ${ }^{4}$, B. E. Tucker ${ }^{5}$, R. J. Foley ${ }^{6}$, W. M. Wood-Vasey ${ }^{7}$, P. Challis ${ }^{2}$, C. Stubbs ${ }^{2,3}$, R. P. Kirshner ${ }^{2}$,
} C. Aguilera ${ }^{8}$, A. C. Becker ${ }^{9}$, S. Blondin ${ }^{10}$, A. Clocchiatti ${ }^{11,26}$, R. Covarrubias ${ }^{9}$, G. Damke ${ }^{12}$, T. M. Davis ${ }^{13}$, A. V. Filippenko ${ }^{14}$, M. Ganeshalingam ${ }^{14}$, A. Garg $^{2}$, P. M. Garnavich ${ }^{15}$, M. Hicken ${ }^{3}$, S. W. Jha ${ }^{16}$, K. Krisciunas ${ }^{17}$, B. Leibundgut ${ }^{18}$, W. Li ${ }^{14,27}$, T. Matheson ${ }^{1}$, G. Miknaitis ${ }^{19}$, G. Pignata ${ }^{20}$, J. L. Prieto ${ }^{21}$, A. G. Riess ${ }^{4,22}$, B. P. Schmidt ${ }^{5}$, J. M. Silverman ${ }^{23}$, R. C. Smith $^{7}$, J. Sollerman ${ }^{24}$, J. Spyromilio ${ }^{18}$, N. B. Suntzeff ${ }^{17}$,

\section{J. L. TONRY ${ }^{25}$, AND A. ZENTENO ${ }^{8}$}

${ }^{1}$ National Optical Astronomy Observatory, 950 North Cherry Avenue, Tucson, AZ 85719, USA; gnarayan@ noao.edu

${ }^{2}$ Harvard-Smithsonian Center for Astrophysics, 60 Garden Street, Cambridge, MA 02138, USA

${ }^{3}$ Deptartment of Physics, Harvard University, 17 Oxford Street, Cambridge, MA 02138, USA

${ }_{5}^{4}$ Space Telescope Science Institute, 3700 San Martin Drive, Baltimore, MD 21218, USA

5 The Research School of Astronomy and Astrophysics, Australian National University,

Mount Stromlo Observatory, via Cotter Road, Weston Creek, ACT 2611, Australia

${ }^{6}$ Department of Astronomy, University of Illinois at Urbana-Champaign, 1002 W. Green Street, Urbana, IL 61801, USA

${ }^{7}$ Department of Physics and Astronomy, University of Pittsburgh, Pittsburgh, PA 15260, USA

${ }^{8}$ Cerro Tololo Inter-American Observatory, National Optical Astronomy Observatory, Casilla 603, La Serena, Chile

${ }^{9}$ Department of Astronomy, University of Washington, Box 351580, Seattle, WA 98195-1580, USA

${ }^{10}$ Aix Marseille Université, CNRS, LAM (Laboratoire dÁstrophysique de Marseille) UMR 7326, F-13388, Marseille, France

${ }^{11}$ Pontificia Universidad Catolica de Chile, Instituto de Astrofisica, Casilla 306, Santiago 22, Chile

${ }_{12}$ Department of Astronomy, University of Virginia, Charlottesville, VA 22904-4325, USA

${ }^{13}$ School of Mathematics and Physics, University of Queensland, Brisbane, QLD 4072, Australia

${ }^{14}$ Department of Astronomy, 601 Campbell Hall, University of California, Berkeley, CA 94720-3411, USA

${ }^{15}$ Department of Physics, University of Notre Dame, 225 Nieuwland Science Hall, Notre Dame, IN 46556-5670, USA

${ }^{16}$ Department of Physics and Astronomy, Rutgers, The State University of New Jersey, Piscataway, NJ 08854, USA

${ }^{17}$ Department of Physics and Astronomy, Texas A \& M University, College Station, TX 77843-4242, USA

${ }^{18}$ European Southern Observatory, Karl-Schwarzschild-Strasse 2, D-85748 Garching, Germany ${ }^{19}$ Fermilab, P.O. Box 500, Batavia, IL 60510-0500, USA

${ }^{20}$ Departamento de Ciencias Fisicas, Universidad Andres Bello, Avda. Republica 252, Santiago, Santiago RM, Chile

${ }^{21}$ Astronomy Nucleus, Faculty of Engineering, Universidad Diego Portales, Ejército 441, Santiago, Santiago RM, Chile

${ }^{22}$ Johns Hopkins University, 3400 North Charles Street, Baltimore, MD 21218, USA

${ }^{23}$ Department of Astronomy, University of Texas, Austin, TX 78712-0259, USA

${ }^{24}$ Oskar Klein Centre, Department of Astronomy, AlbaNova, Stockholm University, SE-10691, Stockholm, Sweden

${ }^{25}$ Institute for Astronomy, University of Hawaii, 2680 Woodlawn Drive, Honolulu, HI 96822, USA ${ }^{26}$ Millennium Institute of Astrophysics, Chile

Received 2015 May 26; accepted 2015 December 22; published 2016 May 6

\begin{abstract}
The ESSENCE survey discovered 213 Type Ia supernovae at redshifts $0.1<z<0.81$ between 2002 and 2008 . We present their $R$ - and $I$-band photometry, measured from images obtained using the MOSAIC II camera at the CTIO Blanco, along with rapid-response spectroscopy for each object. We use our spectroscopic follow-up observations to determine an accurate, quantitative classification, and precise redshift. Through an extensive calibration program we have improved the precision of the CTIO Blanco natural photometric system. We use several empirical metrics to measure our internal photometric consistency and our absolute calibration of the survey. We assess the effect of various potential sources of systematic bias on our measured fluxes, and estimate the dominant term in the systematic error budget from the photometric calibration on our absolute fluxes is $\sim 1 \%$.
\end{abstract}

Key words: cosmology: observations - methods: data analysis - supernovae: general - surveys

\section{INTRODUCTION}

We present the calibrated photometry of 213 Type Ia supernovae (SN Ia) measured by the Equation of State: Supernovae trace Cosmic Expansion (ESSENCE) survey between 2002 and 2008. Our report more than doubles the sample presented by Miknaitis et al. (2007) and Wood-Vasey et al. (2007). We have made a significant effort to improve the photometric calibration of the survey. As ESSENCE observed in only two passbands, our measurements of luminosity distance are strongly correlated with extinction in the host galaxy of the SN Ia and are very sensitive to the systematic error budget from photometry. In particular, the light curves in this work are computed using data taken only with the $4 \mathrm{~m}$

\footnotetext{
27 Deceased 2011 December 12.
}

Blanco Telescope at the Cerro Tololo Inter-American Observatory, eliminating cross-telescope systematics present in the calibration by Miknaitis et al. (2007). A companion work (B. E. Tucker et al. 2014, in preparation) will report on properties of the host galaxies of our SN Ia sample. In future work, we will use this sample along with low-redshift SN Ia from the literature to perform a full cosmological analysis and improve constraints on the nature of the dark energy.

Since the discovery of the luminosity-width-color relation (Phillips 1993), SN Ia have been our most precise standardizable candles at cosmological distances. The initial CalánTololo sample of $29 \mathrm{SN}$ in 4 colors (Hamuy et al. 1996) enabled the development of various algorithms capable of correcting the dispersion in the intrinsic brightness of SN Ia, and inferring the luminosity distances to $\sim 10 \%$ per object 
(Riess et al. 1996; Phillips et al. 1999; Goldhaber et al. 2001). These light curve fitters have been refined as the size of the nearby sample has increased and its photometric precision has improved; current algorithms can determine the luminosity distance to well observed SN Ia to 5\% (Guy et al. 2007; Jha et al. 2007; Conley et al. 2008; Mandel et al. 2011).

The distance moduli derived for these SN Ia indicated that the universe is accelerating (Riess et al. 1998; Perlmutter et al. 1999). SN Ia observations have remained our most sensitive cosmological probe of the expansion history. The accelerating expansion has been modeled by introducing a fluid with negative pressure, called the dark energy, into the Friedmann equation:

$$
h(a)^{2}=h_{0}^{2}\left(\frac{\Omega_{M}}{a^{3}}+\frac{\Omega_{\nu}}{a^{4}}+\frac{\Omega_{k}}{a^{2}}+\Omega_{\mathrm{DE}} \exp [3(1+w)]\right),
$$

where $h$ is the Hubble parameter, $h_{0}=H_{0} / 100 \mathrm{~km} \mathrm{~s}^{-1} \mathrm{Mpc}^{-1}$, $a$ is the scale factor, and $\Omega$ is the total energy density of matter $(M)$, photons $(\nu)$, curvature $(k)$, and the dark energy (DE), respectively. Several groups have focused on measuring the ratio of pressure to density - the equation of state of this fluid, $w=P /\left(\rho c^{2}\right)$ - to distinguish between different models of the dark energy.

High-redshift SN Ia surveys (Riess et al. 2007; Wood-Vasey et al. 2007; Guy et al. 2010; Betoule et al. 2014; Sako et al. 2014) have independently reported measurements of $w$ consistent with -1 , in good agreement with a classical cosmological constant. However, despite the rapidly growing number of SN Ia, the precision of the measurement of $w$ has stubbornly remained at the $10 \%$ level, dominated by various sources of systematic uncertainty. Several groups have attempted to reduce the effect of systematic errors in SN Ia measurements on the dark energy figure of merit (FoM; Albrecht et al. 2006), by either incorporating new sources of data, or improving the calibration of existing data.

Early work by Krisciunas et al. (2000) demonstrated uniformity in the evolution of near infrared (NIR) colors of SN Ia, and the potential of NIR measurements for cosmology (Krisciunas et al. 2004). Using increasingly large and better calibrated samples of nearby SN Ia with $J H K_{s}$ measurements, Wood-Vasey et al. (2008), Mandel et al. (2009), and BaroneNugent et al. (2012) have shown that the NIR light curves of SN Ia span a smaller range in luminosity than in the optical. Because distance moduli derived from NIR measurements are less susceptible to host galaxy dust absorption, the residual scatter in a Hubble diagram generated from infrared light curves alone is comparable to the scatter derived from light-curveshape-corrected optical data. Consequently, high-z surveys have increasingly attempted to probe further into the rest-frame infrared. Freedman et al. (2009) presented the first IR Hubble diagram to $z \sim 0.7$, but were limited by a relatively small sample size, systematic uncertainties in their photometric calibration, and the difficulty of obtaining IR data at high- $z$, where it is redshifted to even longer wavelengths. Future high-redshift surveys, such as RAISIN (R.P. Kirshner-Hubble Space Telescope (HST) Proposal 13046), will provide valuable highredshift SN Ia measurements that probe the rest-frame NIR.

Kelly et al. (2010) illustrated that in addition to demographic differences between SN Ia from passive and star-forming hosts, the Hubble diagram residuals are correlated with derived host galaxy size and stellar mass. This correlation indicates that the empirical luminosity shape relations employed by SN Ia light curve fitters do not fully account for the spread in intrinsic luminosity. In an effort to reduce this dispersion, Lampeitl et al. (2010) employed a simple linear correction based on host galaxy stellar mass and found an improvement in statistical fit to the SN Ia measurements. Sullivan et al. (2010) used different SN Ia absolute magnitudes for high- and low-mass hosts in their cosmological fits and found a significant improvement in $\chi^{2}$ over using a relation expressed as a function of host galaxy stellar mass.

However, although metallicity, extinction properties, and specific star formation rate correlate with host galaxy mass, the fundamental relation underlying this correlation with $\mathrm{SN}$ Ia luminosity is not well understood. These relations may be an artifact of the treatment of SN Ia color by light curve fitters; Scolnic et al. (2014a) found that the strength of correlation of the host galaxy properties with Hubble residual was reduced by $\sim 20 \%$ when SN Ia are treated as having an intrinsic color scatter for a fixed luminosity distance, rather than an achromatic scatter in peak luminosity. In addition, there are challenges in deriving host galaxy properties from broadband optical photometry at high redshift in a manner that does not introduce additional systematic uncertainty into SN Ia measurements. ESSENCE has undertaken a significant effort to determine host galaxy morphology and properties for our sample, to appear in B. E. Tucker et al. (2014, in preparation).

Several authors (Wang et al. 2009; Blondin et al. 2011; Foley \& Kasen 2011; Nordin et al. 2011; Walker et al. 2011; Silverman et al. 2012) have found that measurements from spectra of SN Ia correlate with the residual intrinsic color dispersion after light curve shape correction. They further find that these measurements, typically derived from pseudo equivalent widths of $\mathrm{Ca}$ or $\mathrm{Si}$, can be used to improve the precision of distance moduli, although Blondin et al. (2011) find that the improvement is not statistically significant $(<2 \sigma)$. While promising, this approach is limited by the need for high signal-to-noise ratio $(\mathrm{S} / \mathrm{N})$ spectra of $\mathrm{SN}$ Ia. Additionally, the dependence on measuring the Si II $6355 \AA$ feature limits its use at high- $z$, where the redshifted $S i$ features are often not covered by the high-throughput, low-dispersion spectrographs used by SN Ia surveys.

Surveys such as ESSENCE, the Supernova Legacy Survey (SNLS), the Sloan Digital Sky Survey (SDSS), and the Panoramic Survey Telescope and Rapid Response System (Pan-STARRS) have now produced well over a thousand well sampled SN Ia light curves that span the redshift range over which the transition from cosmic deceleration to acceleration occurred. The crucial measurement for characterizing the nature of dark energy is mapping out luminosity distance versus redshift to constrain the parameters of Equation (1). The precision of photometric calibration is now the dominant term of the SN Ia survey systematic error budget. Wood-Vasey et al. (2007) found that systematic uncertainties from the photometry alone could lead to an $\sim 4 \%$ change in $w$. Exploiting the improved statistics from these large samples requires a corresponding improvement in the photometric calibration across diverse instruments, detectors and filters.

The most important aspect of this calibration challenge is to establish a well grounded understanding of flux measurements made in different broad optical passbands. This in turn requires adopting a spectrophotometric standard, which serves as the metrology basis for relating fluxes across the bands being used. In essence, we need to be able to distinguish cosmological 
evolution in luminosity-distance-redshift relation from crossband calibration issues.

There are two methods in use or in development for flux calibration at CCD wavelengths.

1. Adopt an astrophysical source, particularly Vega, as a celestial transfer standard, with ground-based blackbody emitters as the fundamental calibration sources. This is a long-standing method, and serves as the basis for Vegabased magnitudes (Oke \& Schild 1970; Hayes \& Latham 1975), and underpins the Landolt (1992) standard star network

2. Use well-calibrated laboratory standards (such as silicon photodiodes from NIST) as the foundational metrology layer, and measure the system throughput in comparison to these devices. This was the approach explored advocated by Stubbs \& Tonry (2006) and is now in various stages of implementation by Pan-STARRS (Tonry et al. 2012; Rest et al. 2014), SNLS (Regnault et al. 2009), the joint efforts of SDSS and SNLS (Betoule et al. 2013, 2014), and the Dark Energy Survey (DES) and the Large Synoptic Survey Telescope (LSST).

While the first method is well established, SN Ia surveys require a higher level of precision than is possible with existing standard star networks. The second method is still nascent, and systems to measure the atmospheric component of the throughput are under active development (Albert et al. 2014). No purely laboratory-standard-based magnitude system yet exists. Several surveys, including ESSENCE, have elected to use a combination of both methods; the first to determine the absolute flux calibration, and the second to determine precise relative system throughputs.

Kessler et al. (2009) demonstrated that measurements of $w$ are extremely sensitive to the calibration of the $U$ band at low redshift: inclusion of rest-frame $U$-band data at all redshifts causes a 0.12 mag shift in distance moduli, corresponding to an enormous 0.3 change in the equation of state parameter, $w$. The $U$-band anomaly might arise from differences between the spectral energy distribution (SED) of SN Ia that correlate with host galaxy properties or between objects at low and high redshift (Foley et al. 2012; Maguire et al. 2012). Additionally, $U$-band measurements of the same nearby SN Ia from different telescopes often exhibit differences that are inconsistent with the stated photometric uncertainties and system throughput measurements. Krisciunas et al. (2013) have demonstrated that careful modeling of the $U$-band transmission with appropriate $S$-corrections can resolve the differences between SN Ia measurements. The size of the systematics associated with the $U$-band, however, has led most high- $z$ surveys to down weight, or discard rest-frame UV observations.

Larger, more precisely calibrated nearby samples (Ganeshalingam et al. 2010; Stritzinger et al. 2011; Hicken et al. 2012), along with better calibration of high- $z$ SN Ia surveys, offer the most direct path to reducing the systematic uncertainty on the equation of state parameter of the dark energy. Wide-field deep surveys such as Pan-STARRS and DES will obtain SN Ia measurements over $0<z<1.2$ (Rest et al. 2014), further reducing systematic uncertainties from photometry by avoiding any errors associated with cross-telescope calibration and weakening the sensitivity of $w$ to the overall photometric calibration of the survey (Scolnic et al. 2014b). Recognizing the need for precision calibration to reduce systematics (Stubbs
Table 1

Primary ESSENCE Fields

\begin{tabular}{lccc}
\hline \hline Field & \multicolumn{2}{c}{ R.A. (J2000) decl. } & Number of Images \\
\cline { 2 - 3 } & h m s & $\circ /$ '” & \\
\hline waa & $23: 27: 27$ & $-09: 51: 00$ & 172 \\
wbb & $01: 12: 00$ & $-00: 20: 17$ & 275 \\
wcc & $02: 07: 41$ & $-04: 55: 00$ & 289 \\
wdd & $02: 28: 36$ & $-08: 24: 17$ & 293 \\
\hline
\end{tabular}

\& Tonry 2006; Tucker et al. 2007), and following the example set by the SDSS (Ivezić et al. 2007; Padmanabhan et al. 2008), current surveys have undertaken ambitious calibration programs. These efforts combine high-precision measurements of system throughput calibrated to laboratory standards, with atmospheric data and repeated observations of stellar standards to obtain $<1 \%$ photometry over much of the sky (Stubbs et al. 2010; Schlafly et al. 2012; Tonry et al. 2012). This work details the calibration of the ESSENCE survey, with a focus on minimizing the systematic error budget from photometry.

We provide a brief overview of the ESSENCE survey in Section 2, followed by our photometric data reduction and calibration in Section 3. We discuss our spectroscopic follow up and classification is Section 4. We illustrate our SN Ia light curves, compare and contrast our methodologies for light curve fitting, and detail the properties of the full ESSENCE six-year sample in Section 5. Our photometric error budget from various sources with systematic uncertainty is detailed in Section 6. We conclude in Section 7. The appendices contain further information on the computation of illumination corrections, the properties of the CTIO Blanco natural magnitude system employed in this work, tables containing the photometry of ESSENCE SN Ia and likely SN Ia without spectroscopic confirmation (hereafter, "Ia?") objects during the year of discovery, and light curve fit parameters using the two most common methodologies.

\section{THE ESSENCE SURVEY}

Previous ESSENCE publications have described the survey strategy, fields, data processing (Miknaitis et al. 2007, hereafter M07), spectroscopic selection criteria and follow up (Matheson et al. 2005; Foley et al. 2009), performed a preliminary cosmological analysis (Wood-Vasey et al. 2007, hereafter WV07), and scrutinized exotic cosmological models (Davis et al. 2007). The SN Ia search was carried out on the CTIO 4 m Blanco telescope (hereafter Blanco) over 197 halfnights in dark and graytime between September and January from 2002 to 2008. Science images were obtained using the 64 Mega pixel MOSAIC II camera with an Atmospheric Dispersion Corrector (ADC) through two primary filters (denoted $R$ and $I$ ) similar to Cousins $R_{C}$ and $I_{C}$. The field of view of the system is $0.36 \mathrm{deg}^{2}$ on the sky at the $f / 2.87$ prime focus.

The imager consists of eight $2 \mathrm{k} \times 4 \mathrm{k}$ CCDs arranged in two rows of four. Each CCD is bisected along its length, and each section is read out in parallel, resulting in 16 amplifier images for every science exposure. Readout times are approximately $100 \mathrm{~s}$. Each pixel subtends 0!'27 at the center of the field. Optical distortions cause a radial variance of $\sim 8 \%$ in the pixel scale. 
The survey covered a set of four primary fields (listed in Table 1, together with the number of times each field was observed), each consisting of eight sub-fields, clustered spatially. Fields were selected to be equatorial but outside the Galactic and ecliptic planes, in regions with low Milky Way extinction and minimal IR cirrus, and with coverage from existing surveys (including SDSS, the NOAO Deep Wide-Field Survey, and the Deep Lens Survey) where possible. The fields were spaced to ensure that science images could be taken at low airmass. Fields were divided into two sets and each set was imaged in both filters every other observing night, resulting in a typical cadence of four days. Science frames are exposed for $200 \mathrm{~s}$ in $R$ and $400 \mathrm{~s}$ in $I$. The original $I$ filter (NOAO code c6005) sustained significant damage on 2002 November 10, severely degrading the image quality of $I$-band data in CCDs 1 and 2 (amplifiers 1-4). The filter was replaced on 2003 May 25. CCD 3 failed shortly before the start of the 2003 observing season, resulting in a $12.5 \%$ loss in efficiency until it was replaced in 2004.

Survey images were reduced at CTIO using the "photpipe" pipeline developed for use on the CTIO Blanco by the SuperMACHO survey (Rest et al. 2005; Garg et al. 2007; Miknaitis et al. 2007) that operated contemporaneously with the ESSENCE survey. Each science image was calibrated and aligned with a fixed astrometric grid. We subtracted a reference template for each field, constructed using deep images from previous observations. Point-spread function (PSF) photometry from the resulting difference image was combined to identify sources that had varied over multiple epochs, while eliminating sources of contamination such as difference image artifacts and diffraction spikes from saturated stars. With limited time for spectroscopic follow up observations, we were forced to employ various cuts and selection criteria in order to determine the most promising candidates.

The spectroscopic follow-up observations of ESSENCE candidates is described in Section 4. All candidates were visually inspected to classify them and obtain redshifts. We produced a preliminary reduction of all spectra in real time, using standard IRAF $^{28}$ routines, and some custom IDL routines to facilitate data processing for the various instruments. Estimates of the redshift and classification were obtained on site using SNID (Blondin \& Tonry 2007). When preliminary classifications were unclear, we relied on the experience of the observers to determine if additional spectroscopic follow up was warranted. Fields containing candidates with a clear classification as SN Ia were followed for the remainder of the observing season. Following survey operations, all data were transferred, initially to the Hydra Computing Cluster maintained by the Smithsonian Institution, and later to the Odyssey Compute Cluster hosted by the Research Computing Group at Harvard University for the analysis presented in this paper. All data is also available through the NOAO archive. ${ }^{29}$

\section{DATA REDUCTION}

\subsection{Image De-trending}

The eight CCDs of MOSAIC II were read out in pairs, through two amplifiers per chip, by four Arcon controllers. The

\footnotetext{
28 IRAF is distributed by the National Optical Astronomy Observatory, which is operated by AURA under cooperative agreement with the NSF.

29 http://archive.noao.edu/nsa/
}

cross-talk between the amplifiers is subtracted using the xtalk task from the mscred package for IRAF. All CCD images are de-biased and trimmed and masking was applied to bad pixels and columns. The mask is propagated through all subsequent reduction stages.

All science images are flat-field-corrected using dome flats. These flats accurately corrected for pixel-to-pixel variations but large-scale variations were introduced as a result of uneven illumination of the dome screen and stray light paths in the optical system. While the precision obtained from dome flat images alone is suitable for many projects, we required higher precision for SN Ia cosmology and strived to minimize potential systematic errors in our photometry. We therefore accounted for large-scale illumination variation by constructing an illumination correction from the science images, as described below.

We applied the nightly dome flat image to all science images to construct a temporary preliminary flattened image. The resulting images were masked to remove contamination from all astrophysical sources, normalized to have the same sky value, and then averaged. The derived calibration image was inverted, smoothed with a large kernel, and scaled to have a mean of unity. This illumination correction was applied to the dome flat images to take out residual large-scale gradients. The science images were reprocessed with this final flat-field image.

To estimate the night-to-night stability of the illumination correction, we took the ratio of the correction image between different nights of a single run-a period of time during which MOSAIC II was continuously mounted on the telescope, typically one lunation. We found that the gradient pattern (a representative example is shown in Figure 1) was very stable within a lunar cycle. The standard deviation of the ratio without sigma clipping was typically less that $0.1 \%$, and the absolute value of the maximum difference between the ratio and the average of the ratio image was $<0.003$. Therefore, on nights with few science images of sparse fields or with excess stray light-either from insufficient baffling or around full moonwe exploited the stability of the gradient pattern to estimate the illumination correction from nearby nights. This estimation and temporal stability of the illumination corrections is examined in further detail in Appendix A.

Surveys that use master flats constructed for each run are susceptible to systematic trends, such as long period variations in amplifier gain. By contrast, our procedure avoids such effects: science frames were normalized with nightly flat frames and primarily used illumination corrections determined from the same, or at the least extrapolated only from nearby, nights.

\subsection{Astrometric Calibration}

In order to construct difference images to search for and measure the flux of variable and transient objects, we first imposed a consistent astrometric solution and warped all the science images to a consistent pixel coordinate system. The transformation between the local image pixel coordinate system and the FK5 World Coordinate System is dominated by optical distortions that are well described by a low-order polynomial in radius from the field center. We determined the polynomial terms of the distortion function from images of dense LMC fields using the IRAF task, msctpeak. The distortion terms were used in combination with the IRAF task msccmatch to derive a WCS solution for each field. The distortion terms were 


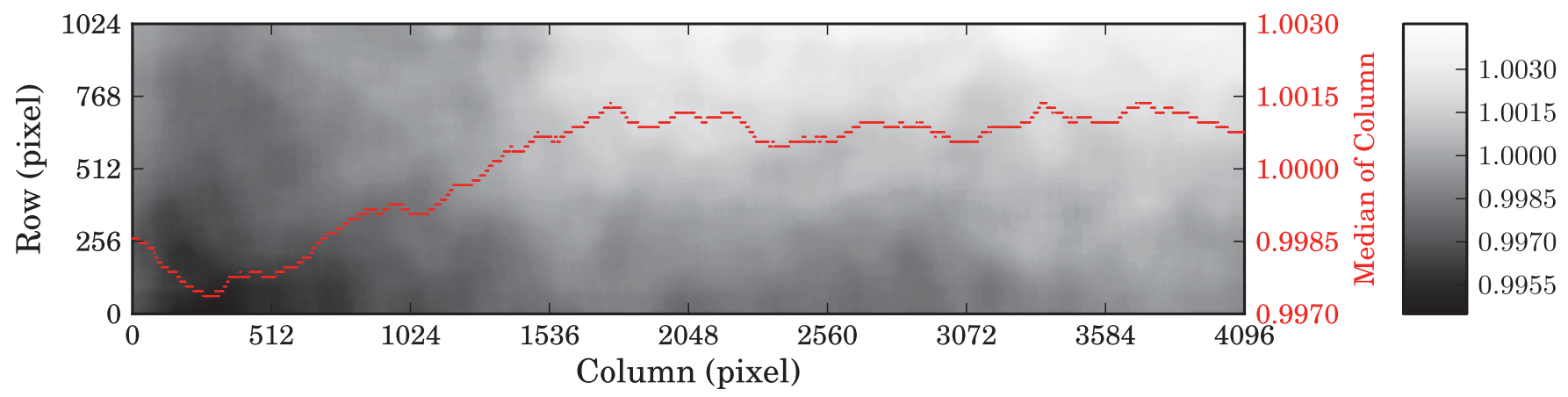

Figure 1. Representative $R$-band illumination correction for amplifier 6 of the MOSAIC II. The primary structure in the illumination correction is a $\sim 0.5 \%$ gradient from left to right and top to bottom. The median value of each column is indicated in red. The bar at right indicates the grayscale values.

re-computed monthly as they vary over timescales of six months. If left uncorrected, this variation would introduce systematic offsets at the $\sim 0$ " 01 level.

With the distortion modeled, the astrometric solution for any image with the equatorially mounted Blanco reduces to determining the linear rotation matrix with respect to the center. We used the IRAF task mscmatch from the mscred package to match pixel coordinates for objects in the image to an existing catalog of the field with precise astrometry. We generated an initial astrometric solution for the survey using reference catalogs derived from the Sloan Digital Sky Survey DR7 (Abazajian et al. 2009) wherever possible, and defaulting to astrometry from the USNO CCD Astrograph catalog 2 (UCAC; Zacharias et al. 2004) where SDSS coverage was unavailable. As the SDSS is itself tied to the UCAC, and as we only require precise relative astrometric calibration to precisely position the PSF and measure flux, errors caused by the differences of the astrometric solution between the two different reference catalogs are negligible. We used this initial solution to generate secondary astrometric catalogs using our multiple observations of each field.

Finally, we used the astrometric solution and the SWarp (Bertin et al. 2002) package to re-sample each image to a common pixel coordinate system using a flux-conserving, Lanczos-windowed sinc kernel. We generated weight maps for each image to account for the change in the noise properties produced by re-sampling. Some covariance between pixels is introduced as a result of the re-sampling process and we accounted for it during difference imaging.

\subsection{Flux Measurement}

We used the DOPHOT photometry package (Schechter et al. 1993) to identify and measure sources within the warped images. DOPHOT is appropriate for point source photometry. B. E. Tucker et al. (2014, in preparation) will report on photometry of extended sources.

\subsection{Photometric Calibration}

High-redshift SN Ia surveys typically report observations in their natural photometric system, relating magnitudes to measured flux via:

$$
m_{T, i}=-2.5 \log _{10}\left(\phi_{\mathrm{ADU}, T, i}\right)+\mathrm{ZP}_{T, i},
$$

where $m$ is the natural magnitude, $\phi$ the measured flux, and $\mathrm{ZP}_{T, i}$ is the instrumental zero point of the image, $i$, observed through passband $T$.
Natural magnitudes have several advantages: they allow surveys to schedule observations in different passbands independently, as the SN Ia colors at every epoch are not needed, and they avoid the additional photometric errors that arise from converting the observed supernova flux to a standard system. These transformations are non-trivial, as the simple linear transformations derived for stars are not directly applicable to SN Ia with their more complex SEDs. However, as these measurements are reported in a non-standard magnitude system, surveys must establish a network of stellar calibrators in the natural system of the telescope to derive accurate and precise zero points. In addition, an accurate model of the survey throughput in each passband is required so measurements in the natural system can be compared to synthetic fluxes generated from models derived from SN Ia measurements at low-redshift in the standard system. We have developed various metrics to quantify our internal photometric consistency, and verified our zero point consistency using the SDSS. We detail the improvements to the photometric calibration for the survey in the next subsections.

\subsubsection{Aperture Corrections}

The extended aureole of astrophysical objects has a surface brightness profile that roughly follows $r^{-2}$, and a large fraction of the flux is outside the seeing disk. Thus, an aperture larger than the seeing disk is necessary for the enclosed flux to be a reliable estimator of the true source flux. However, the larger the aperture, the higher the error from sky subtraction, and the higher the probability of enclosing contaminating sources. We follow the standard technique of addressing this trade off by measuring the flux in a fixed aperture, and determining an aperture correction to correct for its finite size.

Accurate aperture corrections are critical for establishing a consistent photometric system across the survey. We have significantly refined the algorithm used to generate aperture corrections for images. For each sub-field, we identified several isolated objects (typically 10-25 per amplifier) with $\mathrm{S} / \mathrm{N}>20$ that are consistent with a point-source PSF in multiple images. We took care to eliminate instances where we found flux measurements from isolated, but non-stellar objects in the growth curves computed for M07. We measured the flux of each star using aperture radii from 5 to 40 pixels, accounting for the weight map and any flux lost to masked pixels. We constructed differential growth curves for each image (a representative example is provided in Figure 2). The growth curves of individual stars that indicate contamination by a secondary source (cosmic rays, stray reflections, streaks) were 

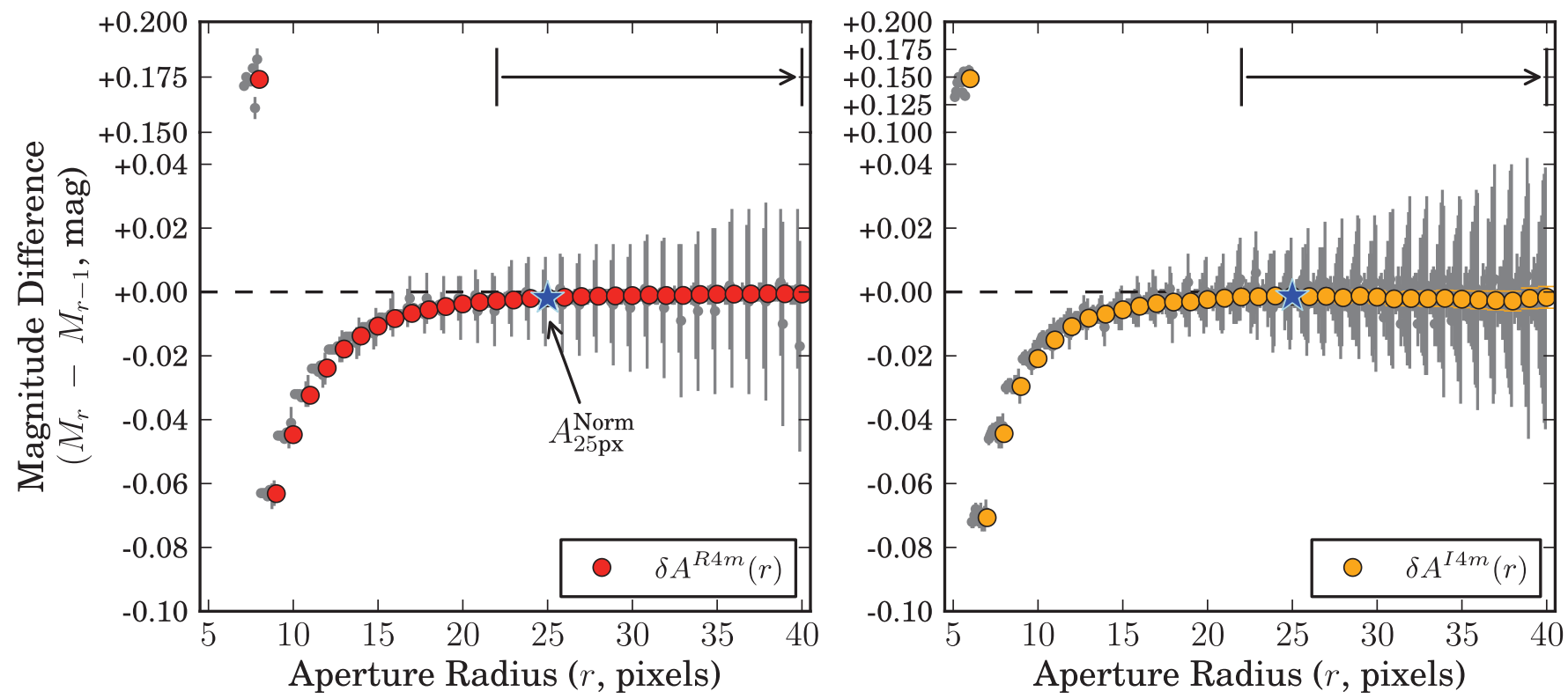

Figure 2. Typical differential curves of growth for $R$ (left, red) and $I$ (right, orange) on 20071103, for amplifier 4 (both randomly selected). The point at the smallest physical aperture is the difference between the DOPHOT magnitude of the object and magnitude with an aperture radius of 5 pixels. We have used a piecewise $y$-axis scale to show the full range of the data without compressing local variations. We have plotted the individual isolated stars in gray. We offset each individual star slightly from the aperture through which the flux is measured along the $-x$ direction for clarity. We checked that the growth curve is consistent with a constant for apertures larger than 22 pixels, indicated by vertical lines with an arrow in between. Errors in the average measurement at each aperture, $\delta A$, are typically smaller than the plot symbols. We propagated the covariance matrix between apertures to determine the final aperture correction at a radius of 25 pixels (indicated with a blue star, and labeled in the left panel).

removed. If more than $25 \%$ of the stars were clipped, the aperture correction for the image was flagged "bad." We checked that the growth curves asymptotically approached a constant value for all apertures larger than 22 pixels, and flagged those that did not. We measured the total aperture correction to an aperture radius of 25 pixels, or $\sim 13$ ". 5 in diameter, chosen to effectively enclose most of the flux of each object for all ESSENCE images, which have a typical FWHM of the PSF of $\sim 1$ !" 2 in both passbands (see Figure 3).

\subsubsection{Choice of Standard Star Network and a Fundamental Spectrophotometric Standard}

While several standard stellar catalogs report broadband magnitudes in different photometric systems through a range of passbands (Landolt 1983; Stetson 2000; Ivezić et al. 2007; Landolt \& Uomoto 2007), the standard star network of Landolt (1992), extended by Stetson (2005), remains the most obvious choice to tie to the Johnson-Morgan-Cousins photometric system. The $R_{C}$ and $I_{C}$ Cousins filters are broadly similar to those used on the Blanco (see Figure 4), and the magnitudes reported by low-redshift SN Ia surveys are converted into the Johnson system using observations of the Landolt network stars. This allows us to minimize systematic uncertainties when comparing our data to the nearby sample.

The choice of standard star network and the transformation equations derived between the natural and standard system also play a critical role in determining the absolute throughput of each passband. This calibration enables SED models of SN Ia generated from low-redshift observations to be converted into the Blanco natural magnitude system via:

$$
m_{T}=-2.5 \log _{10}\left(\int F(\lambda) T(\lambda) \frac{\lambda}{h c} \mathrm{~d} \lambda\right)+\mathrm{ZP}_{T} .
$$

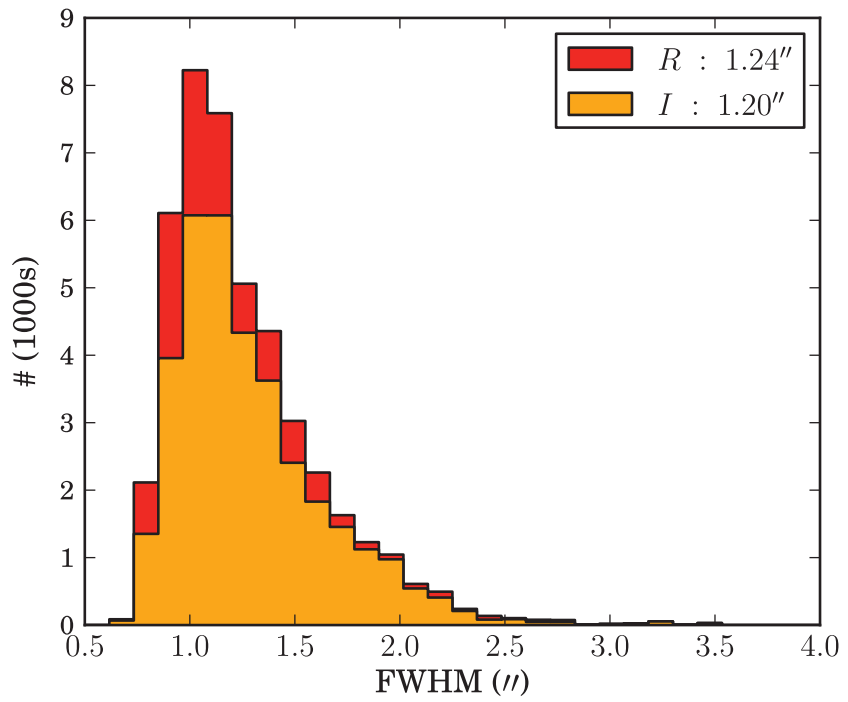

Figure 3. FWHM Distribution of $R$ and $I$ science images from the survey. The mean FWHM is 1 !" 24 for the $R$ band and 1 !. 2 for $I$.

This equation is inverted to determine the zero point, $\mathrm{ZP}_{T}$, for the full optical system (detector, optics, filter, and atmosphere) with dimensionless total photon efficiency, $T(\lambda)$, using a star with a well-measured SED, $F(\lambda)^{30}$, whose magnitudes, $m_{T}$, are known in the natural system —a "fundamental spectrophotometric standard."

\footnotetext{
${ }^{30}$ The formalism employed throughout this work represents SEDs as power per unit wavelength as a function of wavelength, while the system throughput is represented as a dimensionless photon efficiency. The former is typically provided in $\mathrm{erg} \mathrm{s}^{-1} \mathrm{~cm}^{-2} \AA^{-1}$. If the system throughput is provided in erg $\AA^{-1}$, then the extra factor of the inverse energy, $\frac{\lambda}{h c}$, must be dropped to account for the Jacobian of the transformation.
} 


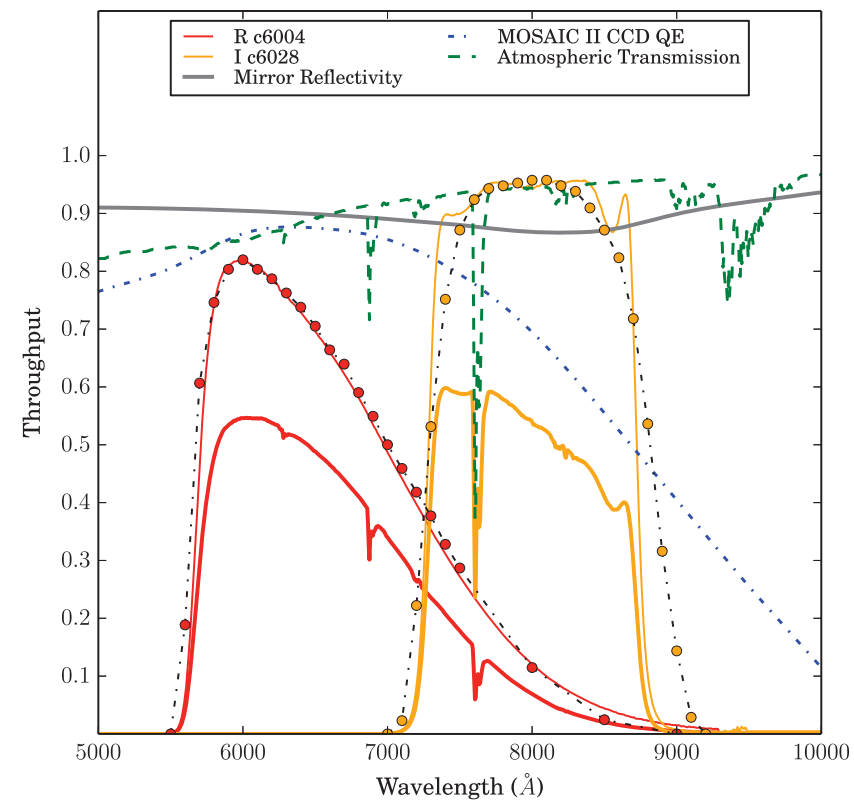

Figure 4. Throughput curves for CTIO Blanco $R$ and $I$ passbands (thick red and orange, respectively), representing full system throughput including wavelength dependence of CCD quantum efficiency (dot-dashed blue), aluminium reflectance of the mirrors (solid gray) in the Blanco telescope, the optical filters (thin red and orange), and a model of the atmosphere (dashed green) generated using the MODTRAN 4 code at an airmass of 1 with $2 \mathrm{~mm}$ PMW of water vapor at an altitude of $2 \mathrm{~km}$, and a contribution from aerosols, appropriate for the CTIO Blanco site. The measurement of the various components of the system throughput is discussed in Appendix B.1, and the response curves are listed in Table 9. The Bessell $R$ and $I$ filter curves (red and orange circles, joined by dot-dashed black lines, and normalized to have the same peak transmission) are shown for comparison.

Unfortunately, most well-measured spectrophotometric standards are too bright to be measured directly by the Blanco. We must therefore infer the Blanco natural magnitudes of the fundamental standard using the star's standard magnitudes. The most direct way of achieving this is to define the transformation equations such that the Landolt and natural system magnitudes agree at some color.

Historically, the choice for the fundamental standard for SN Ia surveys has been $\alpha$ Lyr (Vega), either implicitly when the rest-frame SN Ia model is constructed from low- $z$ data, or explicitly, when defining the passband zero points for highredshift surveys (Astier et al. 2006; Miknaitis et al. 2007; Hicken et al. 2009b, 2012; Contreras et al. 2010; Stritzinger et al. 2011). Vega was one of six A $\varnothing \mathrm{V}$ stars used to establish the color zero point on the photometric system of Johnson \& Morgan (1953) by defining the mean $U-B$ and $B-V$ colors of the six to be zero, and this definition was further extended to Cousins $R_{C}-I_{C}$. Vega's SED was tied to tungsten-ribbon filament lamps and laboratory blackbody sources employed as fundamental standards (Oke \& Schild 1970; Hayes \& Latham 1975). With the widespread adoption of the Landolt standard star network to tie instrumental photometry to the Johnson system, the use of Vega as the fundamental spectrophotometric standard became ubiquitous.

However, as discussed by Regnault et al. (2009), Vega is far from an ideal choice for the fundamental standard. Taylor (1986) found that in order for several sources of synthetic and observed Cousins $R_{C}-I_{C}$ measurements to agree, the $I_{C}$ transmission curve had to be shifted to the red by $50-100 \AA$.
With this shift, the synthetic color of Vega was found to be 0.006 mag. Fukugita et al. (1996) report a similar value. Furthermore, the Landolt $(R-I)_{L}$ color of Vega is significantly more blue than the average for the Landolt standard star network (with $(R-I)_{L} \sim 0.47 \mathrm{mag}$ ), and consequently any systematic error in the color term or the Landolt $(R-I)_{L}$ color of Vega has a much larger systematic effect on the RI natural magnitudes than would a standard with a color closer to the average Landolt standard. Vega may exhibit some photometric variability (Fernie 1981). In addition, its SED is punctuated with several unusually shaped absorption lines. Vega has an excess of NIR emission longwards of 1-2 $\mu \mathrm{m}$, likely a result of its dust ring (Bohlin 2014) and possibly its rapid rotation (Peterson et al. 2006). It also has an excess of UV emission relative to a $9400 \mathrm{~K}$ model (a result of its rapid rotation (Bohlin et al. 2014)). These may introduce systematic errors when models are used to extend the observed SED of Vega into the UV and IR.

Following several groups including the SDSS (Ivezić et al. 2007) and the SNLS (Regnault et al. 2009), we instead select the sdF8 D star, $\mathrm{BD}+17^{\circ} 4708$, as our fundamental spectrophotometric standard. At $(R-I)_{L}=0.32 \mathrm{mag}$, the color of $\mathrm{BD}+17^{\circ} 4708$ is considerably closer to the average Landolt network star than Vega. The HST CALSPEC program has measured the SED of $\mathrm{BD}+17^{\circ} 4708$ covering $0.17-1 \mu \mathrm{m}$ with an uncertainty of $<0.5 \%$ in the flux calibration derived from the three primary HST white dwarf standards and $\sim 2 \%$ in the relative flux calibration over the entire wavelength range.

\subsubsection{Transformation between Landolt Network and the CTIO Blanco Natural System}

In order to calibrate the natural system of the Blanco, we obtained several images of three Landolt standard fields (L92, L95, Ru149) directly with the Blanco/MOSAIC II over 63 nights in 2006 and 2007. The images covered a wide range of airmass and exposure time and the calibration fields were dithered across the entire field of view. With this large data set, we robustly determined extinction and color terms between the Blanco and the Landolt network using the relations

$$
\begin{aligned}
R_{4 \mathrm{~m}}^{\mathrm{Ins}}+A_{i}= & R_{L}+k_{R_{4} \mathrm{~m}}\left(X_{i}-1\right) \\
& +c_{(R-I)_{L}}^{R_{4 \mathrm{~m}}}\left((R-I)_{L}-0.32\right)-Z_{i} \\
I_{4 \mathrm{~m}}^{\mathrm{Ins}}+A_{i}= & I_{L}+k_{I_{4 \mathrm{~m}}}\left(X_{i}-1\right) \\
& +c_{(R-I)_{L}}^{I_{4}}\left((R-I)_{L}-0.32\right)-Z_{i},
\end{aligned}
$$

where $R$ and $I$ denote the $R$ - and $I$-band magnitudes in the Landolt $(L)$ and CTIO Blanco instrumental $(4 \mathrm{~m})$ systems, and $A, X$, and $Z$ denote the aperture correction, airmass, and zero point of an image, $i$ respectively. These relations are defined such that at the color of $\mathrm{BD}+17^{\circ} 4708$, the calibrated magnitudes of the Blanco system match those of Landolt.

We expect differences in the aperture corrections between science and calibration field frames. Images of the calibration fields were generally short exposures $(<60 \mathrm{~s})$ and often unguided, while science images are $200 \mathrm{~s}$ in $R$ and $400 \mathrm{~s}$ in $I$. We found typical systematic differences of $1 \%-3 \%$ between the aperture corrections measured in the calibration fields and the mean aperture correction of all science fields observed on the same nights. The aperture correction differences are correlated with the PSF size and ellipticity measured in the calibration fields. We accounted for these aperture correction differences 
while extrapolating zero points between images to construct the tertiary photometric catalogs in Section 3.5.

The average offset between Landolt magnitudes for catalog stars and measured instrumental magnitudes was calculated for each field, fitting for a single linear term in Landolt $0.3<(R-I)_{L}<0.8$ color. As there were insufficient stars covering the full color range in any single image, the weighted mean color term for all calibration field images with at least 20 stars in $I$ and 50 stars in $R$ was computed. Computing the color term image-by-image allowed us to look for trends in the color term with time and airmass. While this procedure leads to slightly higher statistical uncertainties than if a single color term was determined simultaneously for all images, it produces a robust estimate of the color term and, as shown in Section 6, the systematic uncertainties in the photometric calibration are dominated by the uncertainty in determining the absolute zero points.

We found color terms of $c_{R-I}^{R}=-0.030 \pm 0.001$ and $c_{R-I}^{I}=0.022 \pm 0.001$. These values are in good agreement with measurements by observatory staff ${ }^{31}$ for the Blanco. The dispersion about the fitted value is $\sim 2.5 \%$ in $R$ and $\sim 1.5 \%$ in $I$. While this dispersion is significantly larger than the photon noise, this is not unexpected. We seek a single linear color term that is applicable over a range of color, and in a variety of observing conditions that reflect the conditions under which science images were acquired. As we compute these color terms image-by-image, the dispersion about the mean value reflects unmodeled variation in site conditions, as well as any variation in the sample of stars used to compute the color term for any given image. This procedure is preferable to one in which a subset of images are designated as having been acquired in "perfectly photometric" conditions, and are used for calibration, as any difference between conditions on photometric nights and the mean condition of science nights will lead to systematic errors in the photometric calibration.

The value in $R$ is the same as that used by M07, while we find $c_{R-I}^{I}$ to be lower by $0.008 \pm 0.003$ than that work. We attribute this difference to the different methodology used and the redder color range of stars selected for photometric calibration in the M07 analysis.

Several imagers show a strong radial dependence on the color term. Any relative error in the photometry between the center and periphery of the detector can affect the color term. Such effects can arise because of errors in the illumination correction or chromatic effects. Other wide-field imagers often include devices from different suppliers, and the quantum efficiency, and therefore the color term, is a function of position on the detector. Neither factor is a major consideration for MOSAIC II, and there is no evidence of this effect being a significant concern in other studies with this instrument. Nevertheless, we elected to look for any systematic CCD-to$\mathrm{CCD}$ variation in the color terms. We found that the color term had a standard deviation of 0.005 in $R$ and 0.003 in $I$ about the mean. However, as standard fields were not observed over the full duration of the survey, and we might expect any low-level CCD-to-CCD variation to change as the instrument was mounted, unmounted, and cleaned, we cannot determine if this variance is systematic. Consequently elected to use a single color term for the entire imager, as in M07, and absorb this into our systematic error budget in Section 6.

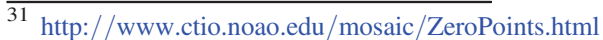

We looked for systematic trends in the residuals between the image-by-image color terms and the mean color term over time, but found that these were not statistically significant. The increasing accumulation of dust on the optical surfaces leads to a changing zero point, but does not significantly affect the color terms.

The offset was re-fit with the color term fixed to this value and the aperture correction was added. Thus, the offset represents the average difference between the Landolt catalog magnitudes and our instrumental magnitudes through a consistent 25 pixel aperture. These aperture-corrected zero points were then regressed against the airmass to determine the slope of the extinction law and intercept.

We found no improvement in allowing the extinction term to vary between survey years. Rather, we found that we could sufficiently account for year-to-year changes in the overall transparency at the CTIO site by decomposing the survey zero point into a dominant constant term with a small night-to-night variation. We measured extinction law slopes of 0.104 mag airmass $^{-1}$ and 0.058 mag airmass $^{-1}$ in $R$ and $I$, respectively, with dispersions of $\sim 0.02 \mathrm{mag}$ about the fitted linear relation. The airmass relation and color terms determined are shown in Figure 5. Additionally, we used the RANSAC algorithm (Fischler \& Bolles 1981) to determine both the extinction and color terms to ensure our fits were not sensitive to outliers. We found differences at the $10^{-5}$ level for the extinction coefficient, and typically at the $10^{-4}$ level for the image-by-image color terms, consistent with the uncertainties on these quantities.

\subsection{Tertiary Catalogs and Zero Points}

Having calibrated the amplifiers within the footprint of the Landolt standard field, we derived an extended standard catalog covering the entire field of view of MOSAIC II. As this catalog was generated by extrapolating the zero point to other amplifiers of the same image, we accounted for the differences in the aperture correction between amplifiers. This procedure prevented any systematic errors arising from PSF variation, a misestimation of the extinction coefficient, or short timescale variations in transparency from affecting the extended standard catalog.

The zero points were then re-determined using the extended catalog without any additional color correction applied. We extrapolated these zero points to science images on the same nights as the calibration images, adjusting for differences in exposure time, aperture correction, and airmass. For each star in the science fields, we determined the $3 \sigma$-clipped, errorweighted mean magnitudes to generate our final photometric reference catalog for each field. Stars with a high rms scatter relative to their mean magnitude errors were rejected as variable. The resulting catalogs typically have $\sim 30$ stars per amplifier, with at least 3 observations in both filters, and a median of 8 observations each in $R$ and 5 in $I$. A $0.4 \%$ uncertainty was added in quadrature to all stars, in order to make the average reduced $\chi^{2}$ unity. The error-magnitude distribution of the reference catalog stars is shown in Figure 6.

These reference catalogs were used to determine zero points for all science images. To examine the temporal stability of the zero points, we adjusted them for differences in aperture correction, airmass, and exposure time, but not nightly variations in transparency or variation between different 

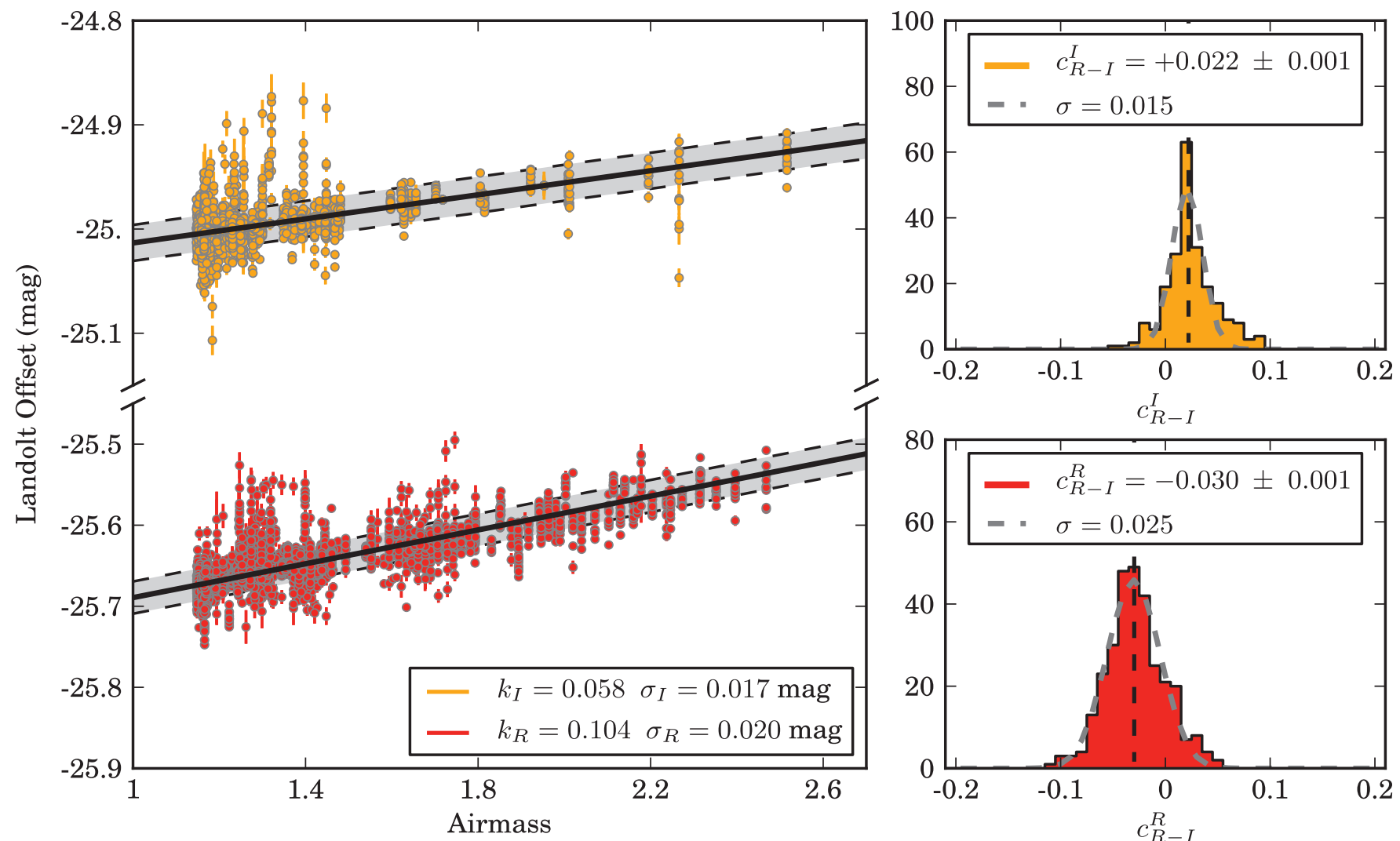

Figure 5. Left: Extinction relation for CTIO Blanco system in $R$ and $I$ using calibration data for three Landolt fields (L92, L95, and Ru149) imaged during the 2006 and 2007 observing seasons. The vertical axis is the difference between instrumental aperture magnitudes, and Landolt catalog magnitudes, corrected for exposure time and variation with Landolt $R-I$ color. We exclude any data taken in non-photometric conditions. We find extinction law slopes of 0.104 mag airmass ${ }^{-1}$ and 0.058 mag airmass $^{-1}$ in $R$ and $I$, respectively. Right: Distribution of color terms, determined per-image, to Landolt $R-I$ for the CTIO Blanco system in $I$ (above) and $R$ (below), using calibration data from 2006 to 2007 . Only images with at least 50 stars in $R$ and at least 20 stars in $I$ were used in the analysis. As there are typically insufficient stars spanning the full color range in any single image, the weighted mean color term for all the images is computed (indicated by dashed vertical lines) and used for all further analysis. We find color terms of $c_{(R-I) L}^{R_{4} \mathrm{~m}}=-0.030 \pm 0.001$ and $c_{(R-I) L}^{I_{4} \mathrm{~m}}=0.022 \pm 0.001$.
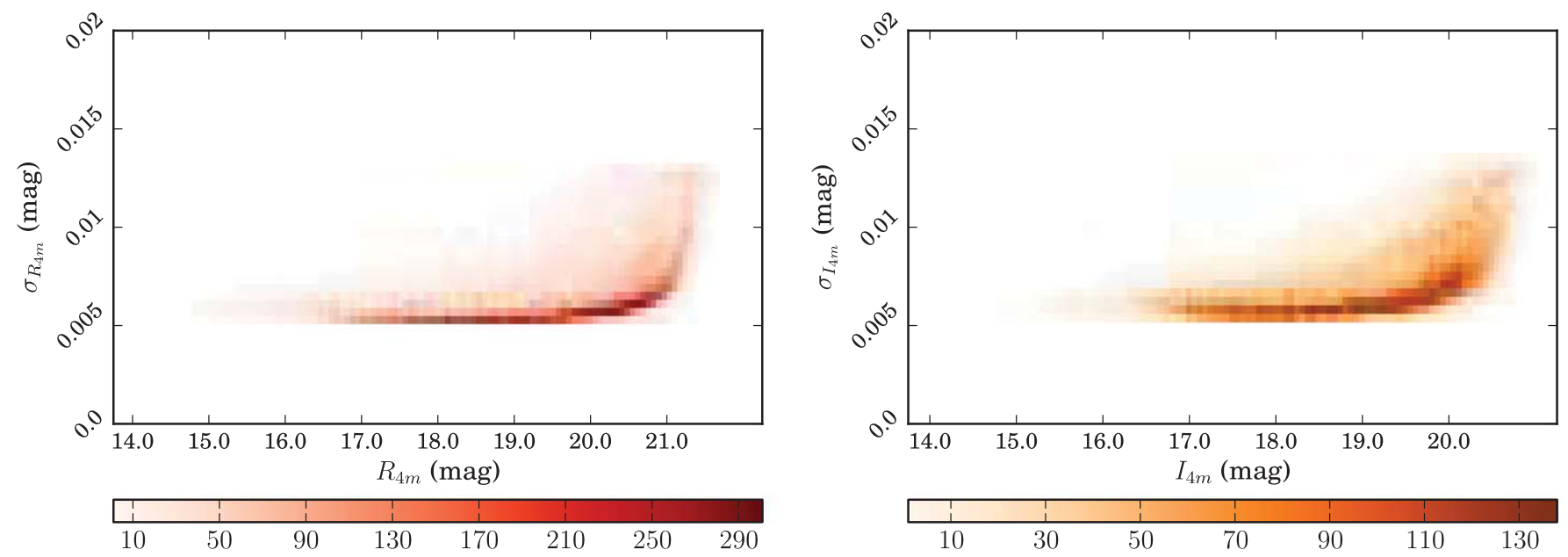

Figure 6. Uncertainty in the Blanco photometry of ESSENCE reference catalog stars as a function of magnitude for $R$ and $I$. The color of each bin indicates the number of stars in that bin. Individual stars require at least three measurements in each filter. The systematic error arising from an error in the airmass or color term, determined in Section 6, has been added in quadrature with the statistical uncertainties.

amplifiers. The adjusted zero points of all available amplifiers were averaged together to construct the average adjusted zero point for a given image. In Figure 7, we plot this quantity as a function of the time since the start of the each year's observing season: the conditions at the Blanco remained very stable over the entire duration of the survey. We also constructed the nightly average zero point, and the histogram of residuals to the nightly average zero point is plotted in Figure 8 . The residual scatter in the nightly zero point residuals is $<2 \%$ in both $R$ and $I$. 

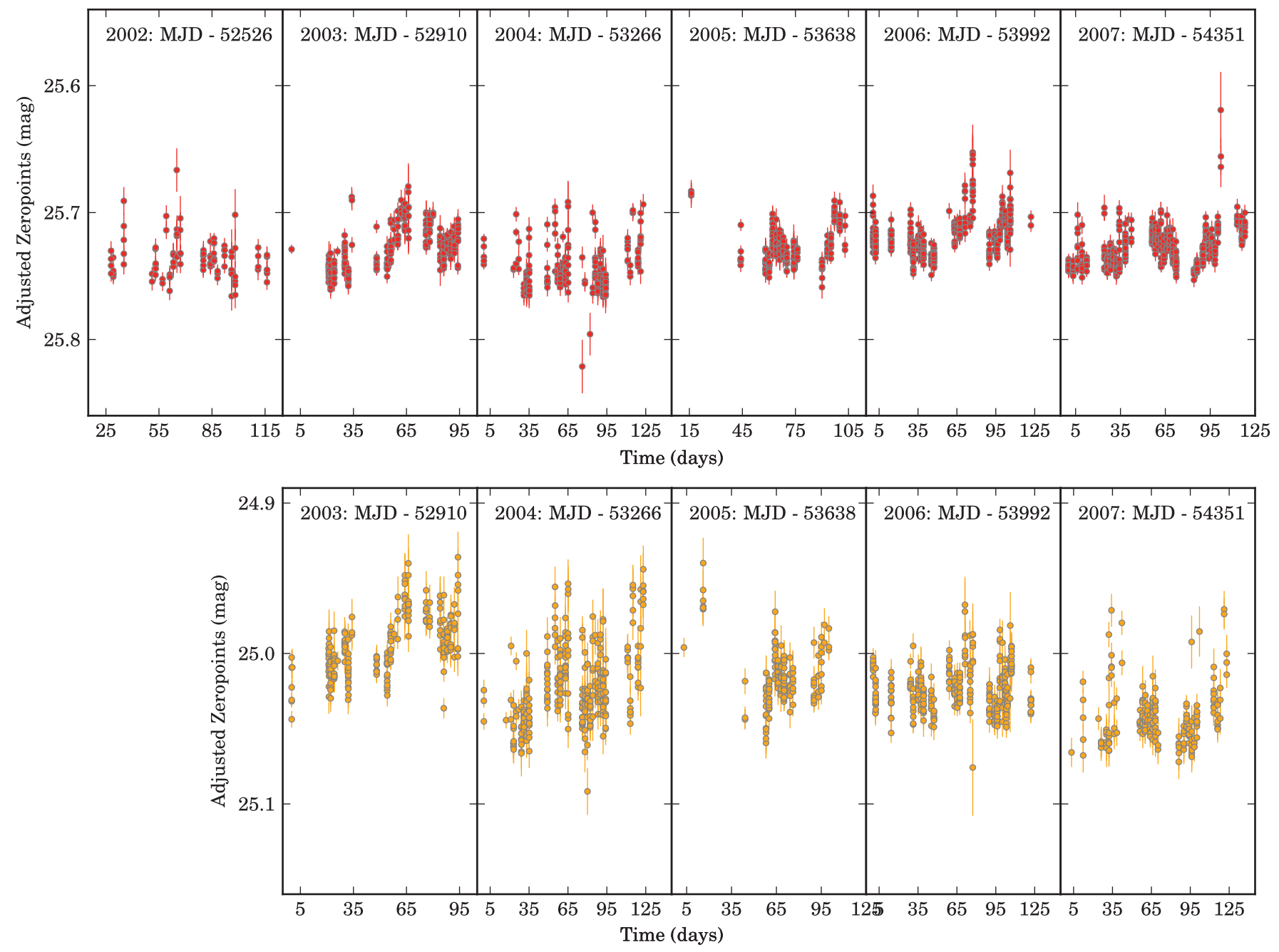

Figure 7. Average zero points for images adjusted for differences in exposure time, aperture correction, and airmass over the full duration of the ESSENCE survey in $R$ (top) and $I$. In 2002, the $I$ filter (NOAO code c6005) was damaged and replaced. The zero point evolution is correlated in both $R$ and $I$, and the short timescale variations correspond to changes in weather conditions at CTIO, whereas the gradual drift in zero points is likely due to the increasing accumulation of dust in the optical system.

\subsection{Image Subtraction}

Having established zero points for each science image, we used image subtraction to remove the background light of the host galaxies. Prior to subtraction, the PSF of each image was first determined from field stars. We used the "High Order Transform Of PSF And Template Subtraction" (HOTPANTS) ${ }^{32}$ package to determine the convolution kernel between each image and template pair. For each pair, the image with the narrower PSF was convolved to match the image with the broader PSF. All $N(N-1) / 2$ possible pairs of image and reference templates from at least three observing seasons were used to create difference images for each object, following the algorithm of Barris et al. (2005). We used a version of DOPHOT (Schechter et al. 1993), modified to use the PSF and flux calibration of the image with the broader PSF, to measure flux in the difference image. The flux calibration of the difference image was adjusted by the normalization of the convolution kernel. The position of the supernova was measured by taking the weighted mean of all detections with a $\mathrm{S} / \mathrm{N}>5$. The

\footnotetext{
32 https://github.com/acbecker/hotpants
}

derived positions are accurate to 0 " 02 . The flux in each difference image was measured with the PSF centroid fixed to the position of the supernova. A representative example of our image subtractions is provided in Figure 9.

As described in $\mathrm{M} 07$, the uncertainties in flux in our difference image are underestimated due to pixel-pixel covariance introduced during the re-sampling process. Rather than scale the noise in each image up by a constant factor of 1.2 , as in M07, we determined a correction for each individual difference image using flux measurements across the frame. We convolved the PSF on a regular grid across the difference image, measured the standard deviation of the distribution of flux $/ \sigma_{\text {flux }}$, and scaled each noise image by this factor. This process effectively accounts for the small residual pixel-pixel covariance introduced by deprojecting each image onto a common astrometric grid, and by the PSF convolution.

Additionally, we constructed a light curve for each object using a single deep reference image, observed in photometric conditions with excellent seeing, to identify any potential problems introduced in processing the thousands of difference images produced by the NN2 process. We found excellent 

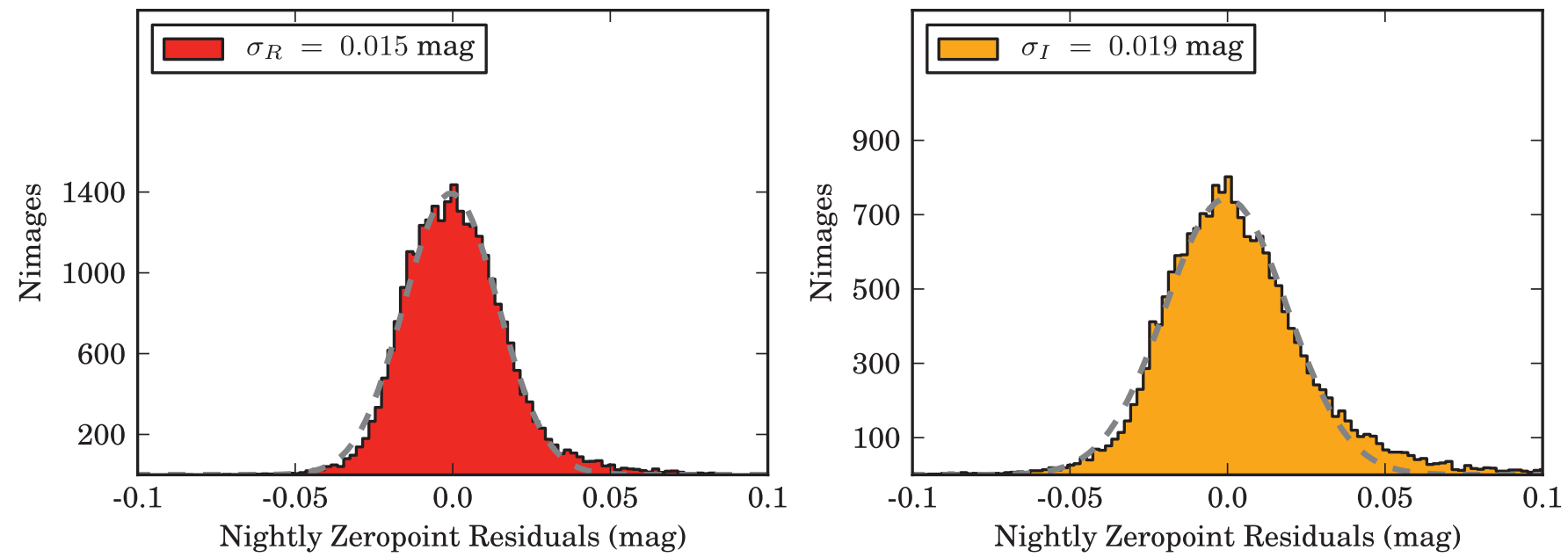

Figure 8. Histograms of the residuals of $R$ (left) and $I$ (right) zero points to the average nightly zero point, adjusted for differences in exposure time, airmass, and the aperture correction. The measured scatter in the nightly zero point residuals is $<2 \%$ in both passbands, consistent with the standard deviations derived from a fit to a Gaussian distribution (dashed gray lines), and very comparable to the values found in M07, illustrating that zero points are very consistent from field to field.
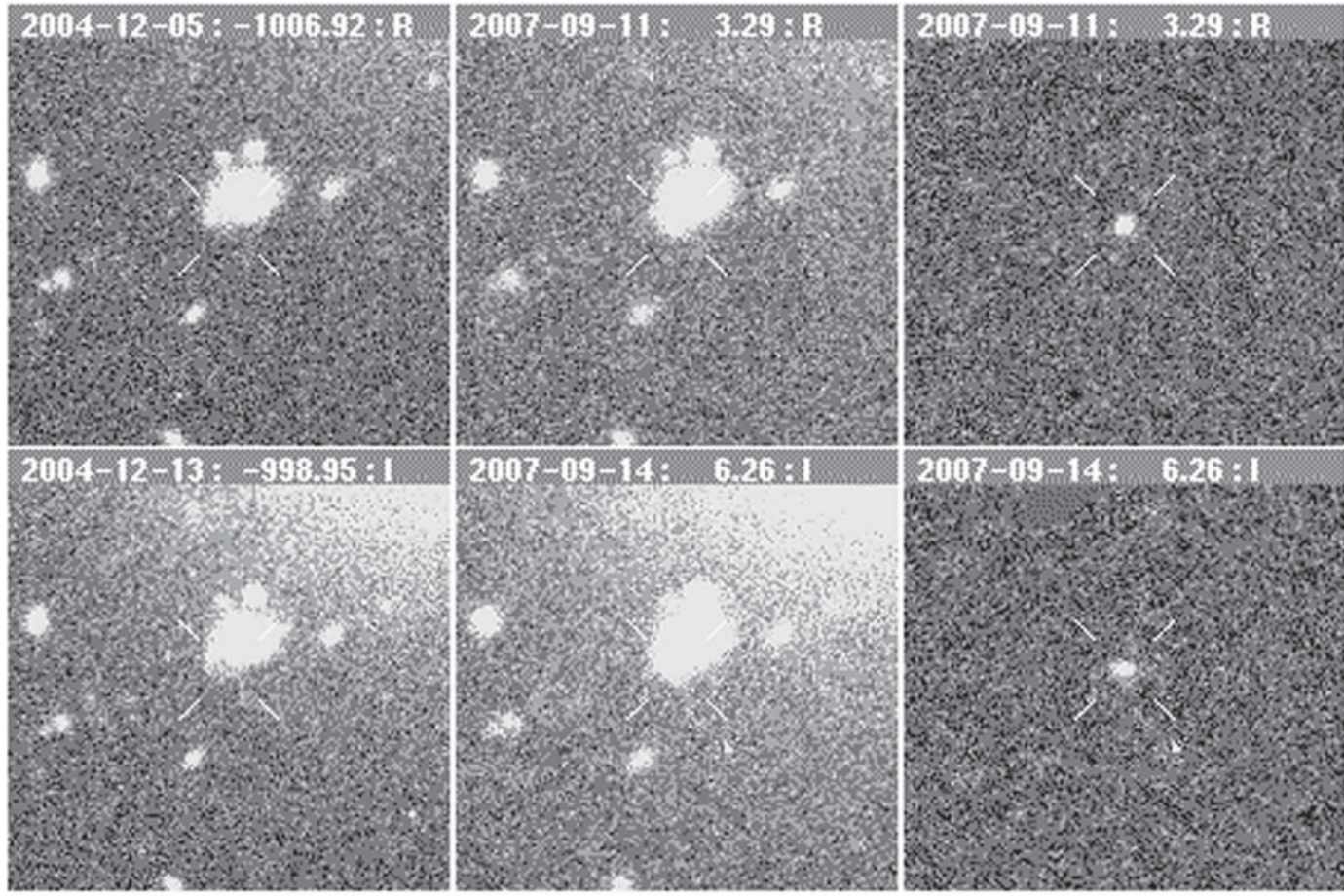

Figure 9. Representative difference imaging "postage stamps" in $R$ (top) and $I$ (bottom) for $x 025$, a SN Ia at $z=0.35$ near the median redshift of the survey. In this instance, HOTPANTS has convolved the PSF of the reference (left) to match the science image (middle). The reference is subtracted to produce the difference image (right). Despite the complex gradient in the background, and clear differences in PSF and depth between the reference and image, the difference image background is extremely uniform and free of artifacts.

agreement between the fluxes measured in the single template and in the NN2 process, with the uncertainty in the flux being lower in the latter, as is expected by the use of multiple images to measure the galaxy template and sky background at each epoch.

\section{SPECTROSCOPY}

Our full sample consists of all SN Ia for which we were able to obtain a positive spectroscopic identification. If possible, slits were aligned to obtain spectra of the host galaxies of the
SN candidates in order to obtain a more accurate redshift. The first two years of spectroscopic data from ESSENCE were presented by Matheson et al. (2005), while M07 detailed our selection criteria and classification algorithms. The spectroscopic observations for the objects included in M07 were presented by Foley et al. (2009). The six-year spectroscopic sample from the ESSENCE survey is presented in this work, together with a summary of the spectroscopic observations, data reduction, and the process of candidate classification and redshift determination. 


\subsection{Selection Criteria for Candidates}

As discussed in Section 2, over the six years of survey operation, ESSENCE detected thousands of objects exhibiting variability over multiple epochs, at a significance of $\mathrm{S} / \mathrm{N}>5$. Given the limited spectroscopic resources for follow up, it was impossible to obtain spectra of all candidates. We employed various selection criteria to narrow the list of candidates from the imaging search to the subset with the most promise of being SN Ia. The first set of these selection criteria was implemented as software cuts in our search pipeline. We required the following.

1. Candidates detected in differences images have the same PSF as stellar objects in the source image that was convolved by HOTPANTS.

2. Candidates exhibit no significant negative flux $(<30 \%$ of the total number of pixels within an aperture of radius $1.5 \times$ FWHM around the detection) to select against difference image artifacts, such as dipoles resulting from slight image misalignment.

3. Candidates did not exhibit significant variability in ESSENCE data from previous years to reject variable stars and active galactic nuclei (AGNs).

4. Candidates in the difference image are not within 1 pixel $(0.27)$ of objects in the template image, as these are frequently AGNs and spectra of such candidates suffer from excessive host galaxy contamination, making classification very uncertain.

5. Candidates exhibit at least two coincident detections with $\mathrm{S} / \mathrm{N}>5$, in at least two passbands or within a five-night window in a single passband, to reject moving objects within the solar system.

6. Detector and image reduction artifacts were excluded by visual inspection.

To select SN Ia from the resulting list of candidates, we fit preliminary light curves using a $B V$ template of a normal SN Ia $\left(\Delta m_{15}=1.1 \mathrm{mag}\right)$ constructed from well sampled low- $z$ SN Ia. This template is a good match to SN Ia observed in $R I$ at $z \sim 0.4$, typical for the ESSENCE survey. Using $\chi^{2}$ minimization, we determined the time of $B$ maximum, the $R I$ magnitudes at maximum, and the light curve stretch, $s$. These factors allowed us to determine an approximate photometric redshift for the object, which, along with the $R-I$ color and rise-time information where available, was used to select likely SN Ia.

An additional level of selection cuts was imposed by the observers on site. Observers tended to favor candidates thought to be in elliptical or low surface brightness hosts, as the former are reliably SN Ia, while the latter aid in extraction of a clean spectrum. As the various facilities and instruments have different capabilities, and reach different depths, our faintest objects were preferentially observed at larger aperture facilities.

We obtained spectroscopic follow up using a range of facilities including the Blue Channel spectrograph on the MMT (Schmidt et al. 1989); IMACS on Baade (Dressler 2004) and LDSS2 (Allington-Smith et al. 1994) and LDSS3 ${ }^{33}$ on Clay at the Las Campanas Observatory; GMOS on Gemini North and South (Hook et al. 2003); FORS1 on the $8 \mathrm{~m}$ Very Large Telescope (VLT) (Appenzeller et al. 1998); and LRIS (Oke et al. 1995), ESI (Sheinis et al. 2002) and DEIMOS (Faber et al. 2003) at the W. M. Keck Observatory.

\footnotetext{
$\overline{33}$ http://www.lco.cl/telescopes-information/magellan/instruments/ldss-3/
}

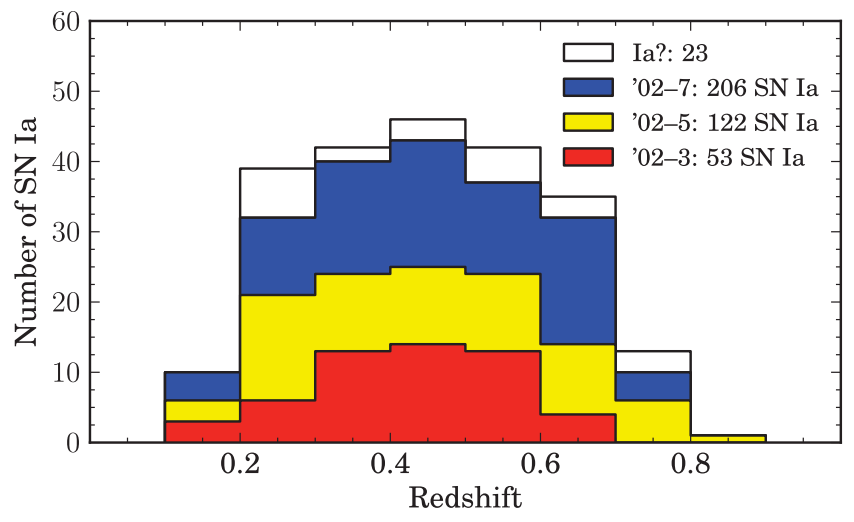

Figure 10. Redshift distribution of spectroscopically identified SN Ia from the ESSENCE survey. Candidates which have a high confidence of being of Type Ia (all objects whose SNID correlations with SN Ia templates exceed $50 \%$ ) are plotted in the shaded region. The histogram is shown for observing seasons spanning 2002-2003 (red), 2002-2005 (yellow), and 2002-2007 (blue), along with cumulative totals, to illustrate the evolution of the redshift distribution over the course of the survey. Candidates for which we have less confidence have been classified "Ia?." Several of these objects have well-measured redshifts from their host galaxies. These are shown in the open region.

Spectra were processed and extracted using standard IRAF routines. Except for VLT data, all spectra were extracted using the optimal algorithm of Horne (1986). VLT spectra were extracted using a novel two-channel Richardson-Lucy restoration algorithm developed by Blondin et al. (2005) to minimize galaxy contamination in the target spectra. Spectra were wavelength calibrated using calibration lamp spectra (usually $\mathrm{HeNeAr}$ ) fit with low-order polynomials, and were fluxcalibrated using a suite of IRAF and IDL procedures, including the removal of telluric lines using the well-exposed continua of spectrophotometric standards.

To avoid relying on subjective assessments of noisy data, we employed the SuperNova Identification (SNID) algorithm (Blondin \& Tonry 2007) to determine SN classifications objectively and reproducibly. SNID is based on the crosscorrelation techniques of Tonry \& Davis (1979). The input spectrum is compared to a large library of template spectra at zero redshift, including nearby $\mathrm{SN}$ of all types (SN Ia, Ib, Ic, II, and subtypes such as SN Ia-pec, SN 91 T, and SN 91bg; see Filippenko 1997 for a review of SN spectral classification), as well as other astrophysical sources such as luminous blue variables (LBVs) and other variable stars, galaxies, and AGNs. Where the redshift of the host galaxy is available, we forced SNID to look for correlations at that redshift $( \pm 0.02)$ to determine the $\mathrm{SN}$ classification. In general, the spectra of $\mathrm{SN}$ with $z>0.5$ have lower $\mathrm{S} / \mathrm{N}$, and thus ambiguities between types occurred mainly in that redshift range.

The SNID algorithm has been presented by Matheson et al. (2005) and Foley et al. (2009), and we refer the reader to these publications for further details.

A list of all objects selected for spectroscopic follow up is provided in Table 6. An analysis of the spectroscopic efficiency of the ESSENCE survey was presented in Foley et al. (2009). The redshift distribution of all ESSENCE SN Ia is shown in Figure 10. 


\section{SN IA LIGHT CURVES FROM THE ESSENCE SIX- YEAR SAMPLE}

Of the 422 objects listed in Table 6, 233 were considered SN Ia candidates based on their preliminary light curves. Spectra were obtained for 229 of those 233 objects. Using the observing spectra, 206 objects have been definitively classified as SN Ia.

Eight objects were observed in non-standard fields, without overlap with the calibration fields used in this paper. Additionally, a few objects were discovered near bright stars, or near the edge of the detector, and suffer from repeated difference imaging failures. We have excluded these objects from further analysis. Despite being classified as a SN Ia (IAUC 8251) ${ }^{34}$, an analysis of the spectra of $e 315$ with SNID indicates that it does not meet the criteria used to classify an object as a Ia employed by this work.

The final RI photometry of 213 of the original 233 candidate SN Ia objects presented in this paper is listed in Table 7. Full light curves, including non-SN Ia objects, and measurements of the baseline flux will be made available as machine readable tables $^{35}$ along with this work. Photometry is presented in linear flux units, $\phi$, in the Blanco natural system for each passband, $T$. Fluxes can be converted to calibrated magnitudes via:

$$
m_{T}=-2.5 \log _{10}\left(\phi_{T}\right)+25 .
$$

The system throughput curves and zero points required to derive magnitudes in our passbands from SED models using Equation (3) are provided in Appendix B. The ESSENCE SN Ia and "Ia?" light curves are illustrated in Figure 11.

\subsection{Light Curve Shape and Color Distributions}

Several different algorithms to fit SN Ia optical photometry exist, including MLCS2k2 (Jha et al. 2007), BayeSN (Mandel et al. 2011), SALT2 (Guy et al. 2007, 2010), SiFTO (Conley et al. 2008), and Dm15 (Prieto et al. 2006). Each of these corrects for the shape and color relations, but they diverge when making two choices: the choice of how to train their spectral models and the choice of how to account for intrinsic and extrinsic color variations. This divergence results in a dichotomy between a physical model, where color variation is decomposed into an intrinsic variance and a reddening, attributed to extinction from dust (MLCS2k2 and BayeSN), versus an empirical model, where all color variation is directly correlated with luminosity (SALT2 and SiFTO). In the following subsection, we compare the color and shape parameter distributions derived using MLCS2k2 and SALT2 for the ESSENCE light curve sample presented in this work.

\subsubsection{Light Curve Quality Cuts}

While all the light curves are fit with both techniques, not all the fits are reliable, as several objects lack high-significance measurements of flux pre- or post-maximum, and these typically exhibit high $\chi^{2}$ /dof. Furthermore, objects in Table 6 without determined redshifts are not fit.

Some selection cuts are common to all SN Ia surveys, and are required to ensure that the light curve fit is well-constrained. These cuts are typically expressed in terms of the rest-frame

\footnotetext{
$\overline{34}$ http://www.cbat.eps.harvard.edu/iauc/08200/08251.html

35 Available through FAS Research Computing at Harvard-http:// telescopes.rc.fas.harvard.edu/index_w.html.
}
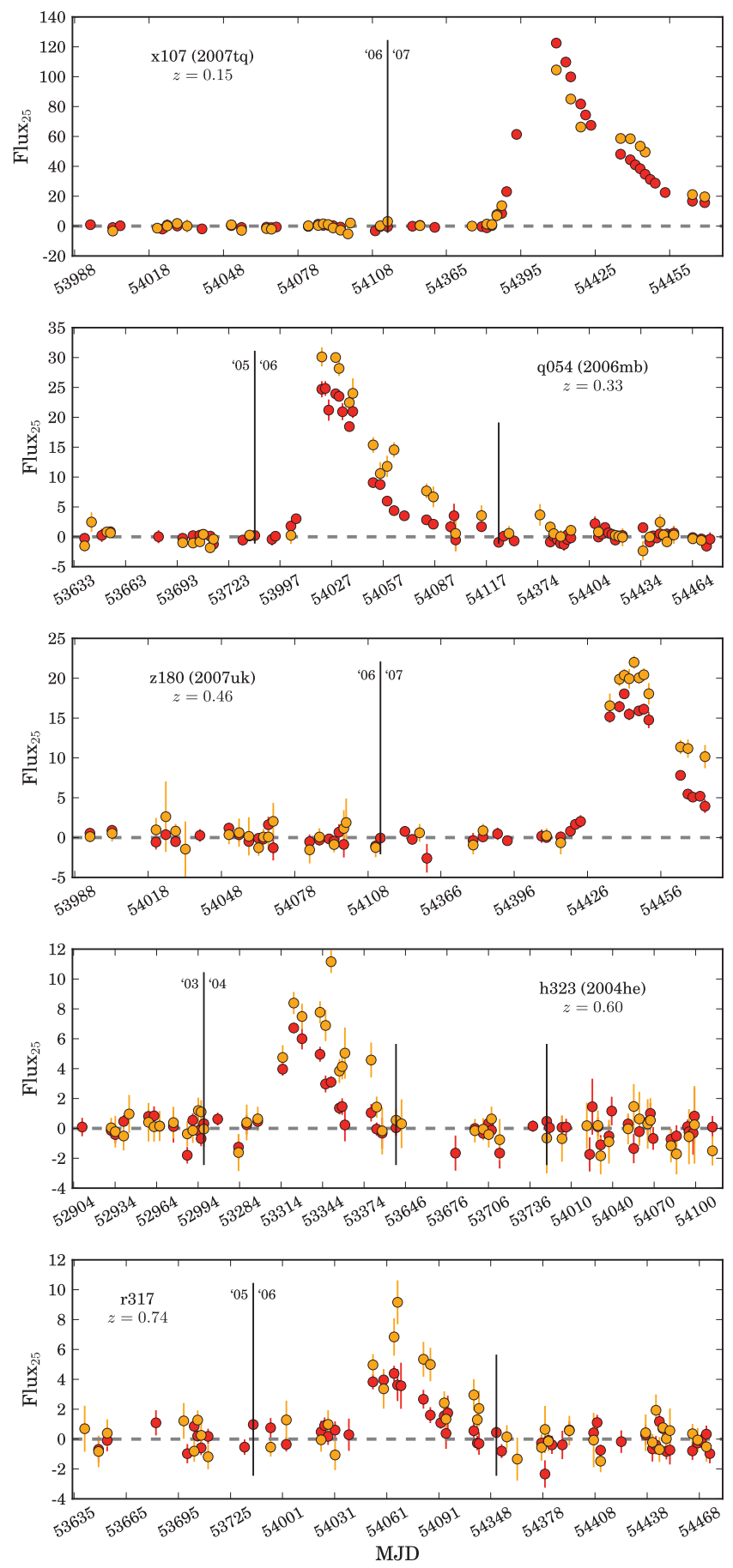

Figure 11. Example ESSENCE $R$ (red) and $I$ (orange) light curves, in units of linear flux, scaled such that a flux of unity corresponds to magnitude 25 . Gaps between observing seasons have been removed, and the reported MJD is discontinuous at the locations of the vertical black lines. The first of these lines is elongated and the year of the observing season is indicated to the left and right of it.

phase in rest-frame days $\Phi=\left(T_{\mathrm{Obs}}-T_{\mathrm{Max}}\right) /(1+z)$. Kessler et al. (2009, hereafter K09) required at least one measurement with $\Phi<0.0$. Guy et al. $\left(2010\right.$, hereafter G10) ${ }^{36}$ employed a more flexible cut, only requiring a single measurement in the

\footnotetext{
36 http://supernovae.in2p3.fr/salt/
} 
range of $-8<\Phi<+5$ days, and found that this provided a comparable constraint to the K09 cut. Similarly, WV07 required at least one observation with $\Phi \leqslant+5$ days for both MLCS2k2 and SALT, but also demanded that the observation had $\mathrm{S} / \mathrm{N}>5$, while requiring that the uncertainty on the fit time of maximum, $\sigma_{T_{\mathrm{Max}}}$, be $<2$ days. The WV07 cut is effective at ensuring that the time and the peak flux are well-constrained, and we adopt it here for ESSENCE SN Ia. The compilation of 441 SN Ia presented by Conley et al. (2011, hereafter C11) uses the weaker G10 cut on observations near maximum. In addition, G10 do not impose any cut on S/N. However, these objects have observations in more passbands than ESSENCE, and the more conservative cut is appropriate.

When the cut on pre-maximum measurements is not applied, both the MLCS2k2 and SALT2 light curve shape parameters ( $\Delta$ and $x_{1}$, respectively) exhibit a significantly increased scatter as a result of light curve fits being ill-constrained with only the post-maximum decline. Scolnic et al. (2014a) also reports that $x_{1}$ shows a trend toward larger values for $z>0.4$ if the premaximum data is excluded. G10 did not find such a trend with high $\mathrm{S} / \mathrm{N} \mathrm{SN}$ Ia at $z<0.4$, illustrating how the effect of light curve quality cuts varies with median redshift, and therefore with survey.

WV07 and K09 also required that the fit statistic, $\chi^{2} /$ dof, be $<3$ for both light curve fitters. C11 did not impose any qualityof-fit cut, as they felt that the reported uncertainties for low- $z$ photometry are frequently inaccurate, rendering such a cut misleading. They also suggested that several light curves contain the occasional outlying photometric observation that drives $\chi^{2}$ dof to artificially high values, despite having little to no effect on the derived light curve shape and color parameters. C11 also argues that any $\chi^{2}$-based cut has an asymmetric effect with an SN Ia sample, and therefore can potentially a introduce bias with redshift. This in turn could lead to systematic bias on $w$. While there is merit in this argument, upon visual inspection of our light curve fits, we concluded that the $\chi^{2} /$ dof statistic did accurately represent the quality of the fit, and that this cut was well motivated. In future work, we will use Monte Carlo simulations to assess any biases in cosmological inference that result from this cut.

Another common cut is on the minimum number of degreesof-freedom. Both WV07 and K09 require $N^{\text {min }}$ dof $\geqslant 5$. C11 do not explicitly state such a requirement, but the compilation they presented nevertheless satisfies that requirement. We adopt $N^{\text {min }}$ dof $\geqslant 5$ for MLCS2k2; however, we found that this cut had the consequence of biasing us toward intrinsically brighter objects. WV07 also required one observation with $\Phi \geqslant+9$ days for MLCS2k2. This cut was intended to ensure that the decline post-maximum is well sampled. As MLCS2k2 also imposes its own cut by requiring observations with $\mathrm{S} /$ $\mathrm{N}>5$, this cut is considerably more stringent than was intended. This requirement causes a total of $44 \mathrm{SN}$ Ia and "Ia?" objects to fail the selection cuts—by far the single largest cut on our MLCS2k2 fits. In addition to eliminating observations of faint sources, or sources at high- $z$ with extremely well sampled declines, the $\mathrm{S} / \mathrm{N}$ cut imposed by MLCS2k2 causes several light curves fits to fail the selection cuts as a result of an insufficient observations, given the requirement of $N^{\mathrm{min}}$ dof $\geqslant 5$ in the MLCS2k2 fit.

By contrast, WV07 only required one observation post- $B$ band maximum for $S A L T$, and only three objects in our sample do not meet this cut. We believe that this demonstrates that
Table 2

Effect of Light Curve Quality Cuts on the ESSENCE Sample

\begin{tabular}{lcc}
\hline \hline Cut & MLCS2k2 & SALT2 \\
\hline Fit Failed & 7 & $\ldots$ \\
$\chi^{2}>3$ & 10 & 20 \\
$N^{\text {min }}$ dof $^{\text {a }}$ & 18 & 20 \\
$\Phi_{\text {First S } / \mathrm{N}>5}{ }^{\mathrm{b}}$ & 9 & 17 \\
$\sigma_{T_{\text {Max }}}$ & 5 & 3 \\
$\Phi_{\text {Last }}$ & 44 & 3 \\
$x_{1} \mid \Delta$ cut & 14 & 18 \\
$c$ cut $^{\mathrm{c}}$ & $\mathrm{NA}$ & 33 \\
\hline
\end{tabular}

Notes. The number of SN Ia and "Ia?" objects that are removed by each selection criterion. Each cut is imposed independently. Many objects fail multiple cuts.

${ }^{\text {a }}$ We require $N^{\text {min }}$ dof $\geqslant 8$ for SALT2, rather than the weaker cut of 5 for MLCS2k2, as the last phase cut is very ineffective with SALT2 when our well sampled NN2 light curves are fit in flux space.

${ }^{\mathrm{b}}$ While at first glance it appears that more objects fail the cut on pre-maximum imaging with SALT2 than with MLCS2k2, this is not the case on closer inspection. MLCS2k2 merely fails catastrophically for objects without premaximum imaging, and consequently does not report $T_{\mathrm{Max}}$ at all.

${ }^{c}$ WV07 did not employ an extinction cut. While we have not used one in this work, it is likely that we will employ a reasonable cut on this value to remove any highly reddened objects at low- $z$ from the sample for a cosmological, as there is considerable uncertainty about the nature of the dust in the host galaxies of highly extinguished SN Ia.

MLCS2k2 is being needlessly conservative by requiring that all observations have $\mathrm{S} / \mathrm{N}>5$. However, the intent of the cut on the number of observations post-maximum is to ensure that the light curve extinction or color is well-constrained, and the location of the peak is bounded. We are wary of the relatively weak effect of the post-maximum cut on our SALT2 light curve fits, and require a stricter $N^{\text {min }}$ dof $\geqslant 8$ for that fitter. With the ESSENCE four-day cadence, this effectively ensures that there are at least four measurements of the observer frame $R-I$ color. As a result, the number of objects that fail the $N^{\text {min }}$ dof cut for MLCS2k2 and SALT2 are similar, and some of the most egregious outliers in $x_{1}$ and $c$ are eliminated.

Based on the results in G10, C11 imposed a restriction on the SALT2 color parameter, and required $-0.25<c<0.25$ mag. WV07 did not explicitly impose an equivalent cut on $A_{V}$ for MLCS2k2. Several groups have used multi-color photometry of highly extinguished low- $z$ SN Ia to demonstrate that the extinction law in the host galaxies of these objects appears to follow the O'Donnell (1994) extinction law with a significantly lower $R_{V}$ than the Milky Way (Hicken et al. 2009a; Folatelli et al. 2010; Mandel et al. 2011).

Additionally, Scolnic et al. (2014b) employs a requirement that $-3<x_{1}<3$ for the Pan-STARRS1 SN Ia sample. Both these cuts are well motivated as there are few SN Ia in the SALT2 training sample outside these ranges, and the fits are likely to be ill-conditioned there. WV07 adopted a requirement of $-0.4 \leqslant \Delta \leqslant 1.7$. All objects in our ESSENCE SN Ia sample that fail this requirement also fail other selection cuts.

A summary of the number of light curves that fail each cut for both MLCS2k2 and SALT2 is provided in Table 2.

\subsubsection{MLCS2k2 Light Curve Analysis}

We employ "v007" of MLCS2k2 with the "tweaked-slowz" vectors. These vectors, and the corresponding matrix of model uncertainties (denoted $S$ ), are trained using the low- $z$ Hubble- 

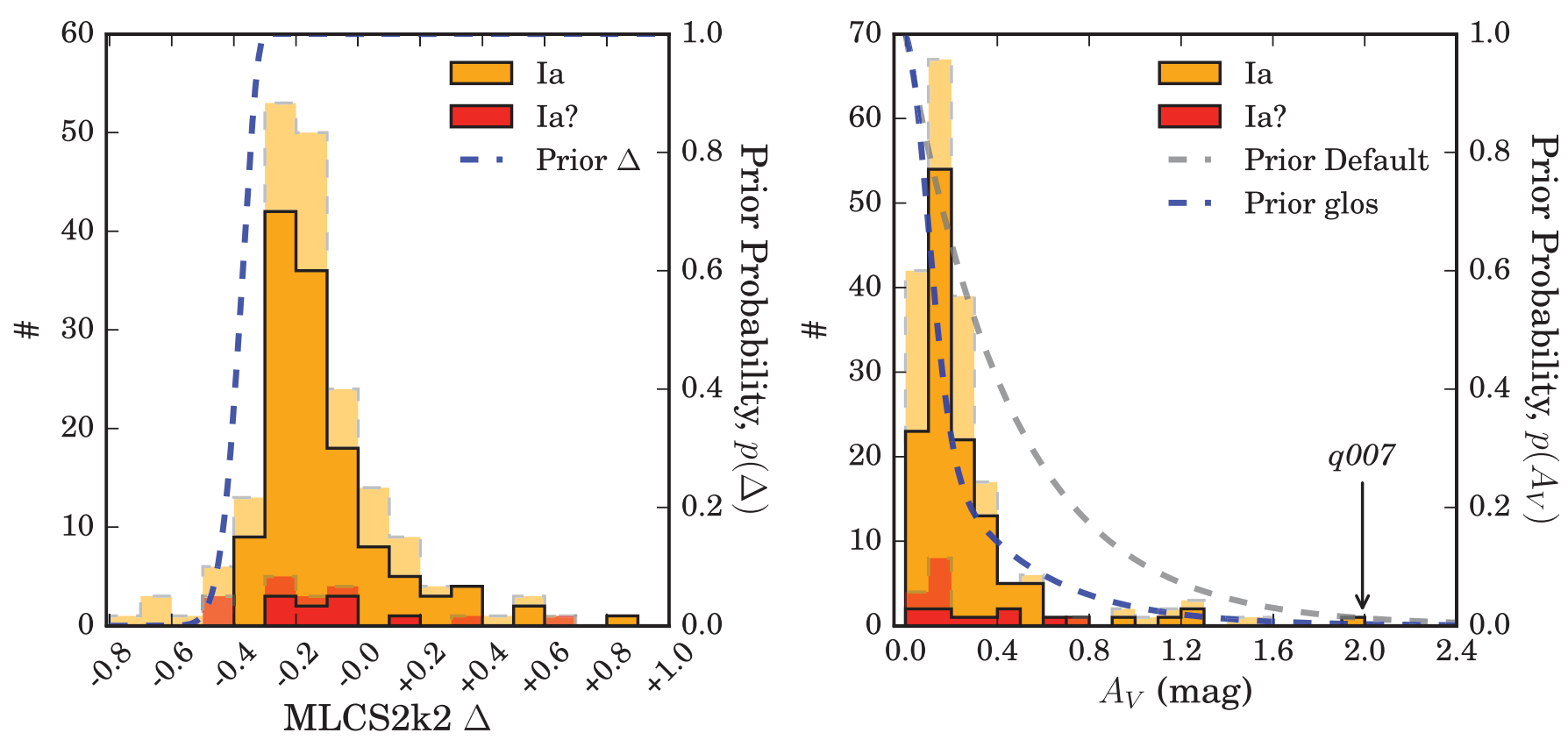

Figure 12. Light curve shape, $\Delta$ (left panel), and extinction, $A_{V}$ (right panel), distributions estimated by MLCS2k2 for ESSENCE SN Ia (orange) and "Ia?" (red) objects. Objects that pass the selection cuts (excepting the cuts on the parameter being plotted itself) imposed in WV07 are indicated in the solid regions, while objects that fail are shown in the light regions bounded by dashed lines. The MLCS2k2 priors employed in the light curve fitting are shown as dashed blue lines. The "default" prior is the extinction distribution derived from low-zSN Ia during the training procedure.

Table 3

MLCS2k2 Light Curve Fit Parameters for ESSENCE SN Ia and "Ia?" Objects

\begin{tabular}{|c|c|c|c|c|c|c|c|c|c|c|c|}
\hline ID & $\mu^{\mathrm{a}}$ & $\sigma_{\mu}$ & $T_{\max }^{B}$ & $\sigma_{T_{\max }}$ & $\Phi_{\text {First }}{ }^{b}$ & $\Phi_{\text {Last }}$ & $\Delta$ & $\sigma_{\Delta}$ & $A_{V}^{\mathrm{c}}$ & $\sigma_{A_{V}}$ & $\overline{Q^{d}}$ \\
\hline e108 & 42.370 & 0.140 & 52979.48 & 0.59 & -11.768 & 9.954 & -0.338 & 0.111 & 0.097 & 0.102 & $\mathrm{~T}$ \\
\hline $\mathrm{k} 425$ & 41.207 & 0.254 & 53335.19 & 0.43 & -9.535 & 19.618 & -0.087 & 0.177 & 0.310 & 0.236 & $\mathrm{~T}$ \\
\hline q002 & 41.212 & 0.359 & 54002.83 & 0.75 & -6.340 & 15.192 & 0.594 & 0.279 & 0.549 & 0.369 & $\mathrm{~T}$ \\
\hline x080 & 42.006 & 0.440 & 54384.94 & 1.54 & -4.178 & 17.699 & 0.132 & 0.343 & 0.315 & 0.276 & $\mathrm{~T}$ \\
\hline
\end{tabular}

Notes.

${ }^{\mathrm{a}}$ MLCS2k2 reports distance moduli with a $H_{0}=65 \mathrm{~km} \mathrm{~s}^{-1} \mathrm{Mpc}^{-1}$

${ }^{\mathrm{b}} \Phi_{\text {First|Last }}$ is the rest-frame phase of the first and last observation, respectively, and is dependent on the $B$ bandtime of maximum, $T_{\max }^{B}$.

' We use the Galactic reddening law of O'Donnell (1994), with $R_{V}$ fixed to 3.1 to model the extinction in the host galaxy of the supernova.

${ }^{\mathrm{d}}$ Flag describing if the object passes (T) or fails (F) the light curve quality cuts described by WV07.

flow sample in Jha et al. (2007; hence "slowz") and "tweaked" with small magnitude offsets (typically $<0.005 \mathrm{mag}$ ) to match the color-extinction distribution zero point, and extended to -20 days prior to $B$-band maximum. We follow WV07 in using the "glosz" prior on extinction, and assume $R_{V}=3.1$.

The MLCS2k2 light curve shape, $\Delta$, and extinction, $A_{V}$, distributions for ESSENCE SN Ia and "Ia?" objects are shown in Figure 12. The MLCS2k2 light curve fit parameters for the ESSENCE sample are provided in Table 3. Additionally, we have indicated if the objects pass the light curve quality cuts used by WV07 for the four-year sample. From our spectroscopically confirmed SN Ia sample, 126 objects pass these cuts and are useful for cosmological inference. This doubles the 4 year ESSENCE sample of $60 \mathrm{SN}$ Ia.

Objects with $\Delta>1.0$ are underluminous relative to normal SN Ia, and are more rare. Consequently, we are extremely unlikely to find any at the redshifts probed by the ESSENCE survey. Objects that appear to be extremely overluminous (very negative values of $\Delta$ ) relative to the training sample of Jha et al. (2007) typically have little or no high-significance flux measurements pre-maximum, but have well-measured declines post-maximum. Without a good constraint on the peak and time of maximum, light curve fitters typically explore unphysical regions of parameter space. The $\chi^{2} /$ dof of these light curve fits is often relatively high $(>3)$ and all fail the quality cuts of WV07, either owing to a high $\chi^{2} /$ dof or because of insufficient observations pre-maximum.

The $A_{V}$ distribution for ESSENCE SN Ia is consistent with the "glos" model discussed employed by WV07. The distribution is significantly narrower than the MLCS2k2 "default" distribution, derived from nearby SN Ia, as we are unlikely to find highly extinguished and therefore faint objects at high- $z$. As MLCS2k2 is a magnitude based fitter, it rejects measurements with $\mathrm{S} / \mathrm{N}<5$. Most of the objects that fail the selection cuts in the right panel of Figure 12 are extremely faint or at high- $z$.

Note that Figure 12 shows the distribution for all recovered fits, and several of these objects do not have light curve fits that meet the quality cuts of WV07. Quality cuts are imposed to select spectroscopically confirmed SN Ia, with several high S/ $\mathrm{N}$ measurements over rest-frame phase $-5 \leqslant \Phi \leqslant 20$ days, to ensure that the derived distance moduli are unbiased, whereas 

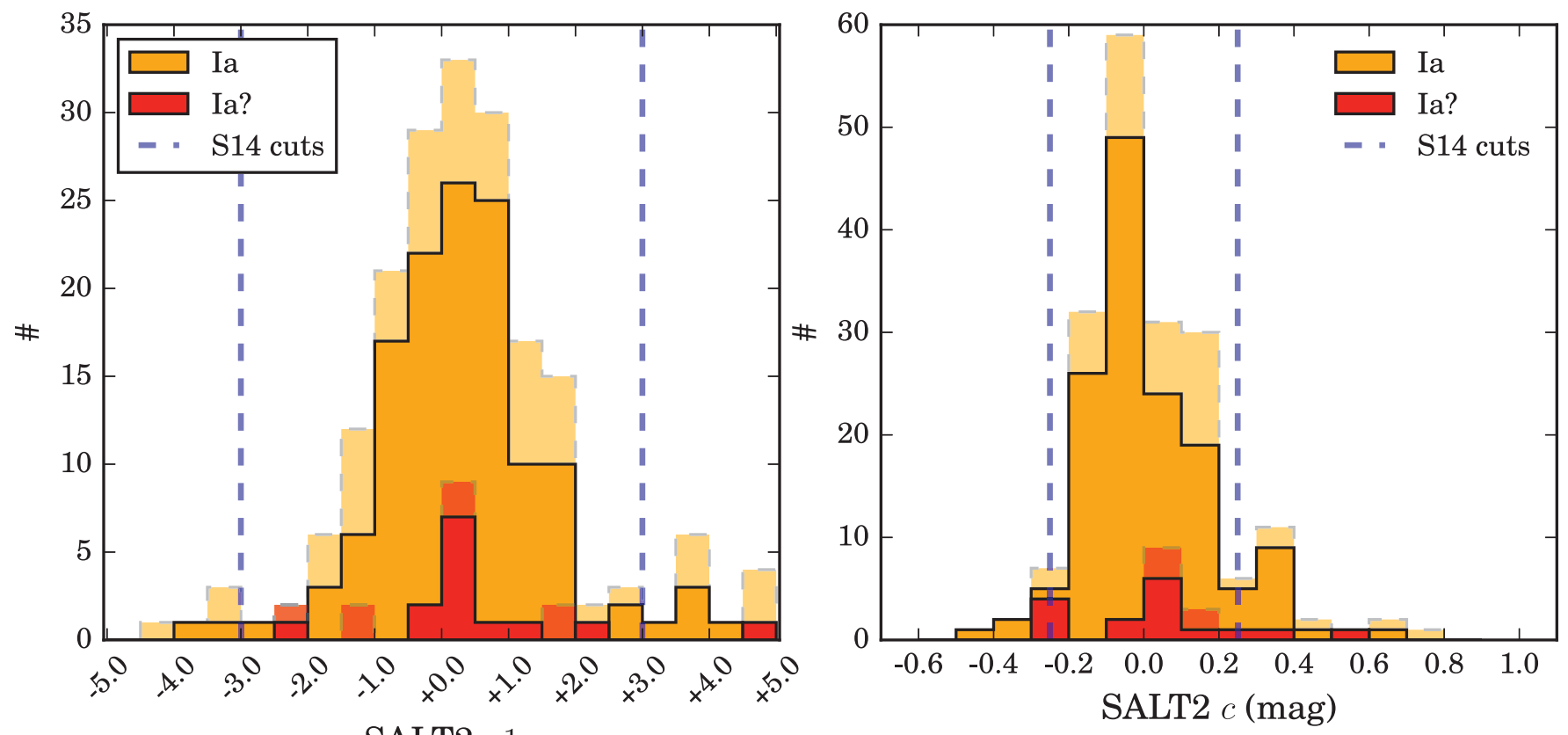

Figure 13. Light curve shape, $x_{1}$ (left panel), and color, $c$ (right panel), distributions estimated by SALT2 for ESSENCE SN Ia (orange) and "Ia?" (red) objects. Objects that pass the original SALT selection cuts (except the cuts on the parameter being plotted itself) imposed by WV07 are indicated in the solid regions, while objects that fail are shown in the light regions bounded by dashed lines. Only the WV07 cuts relating to the sampling and $\chi^{2} /$ dof of the fit are used here. Based on a visual inspection of all the light curve fits, we required that the fits used at least eight epochs, rather than the weaker cut of at least five epochs employed for $S A L T$ in WV07. We employ the same cuts as Conley et al. (2011) and Scolnic et al. (2014a) on $c$ and $x_{1}$, respectively.

Table 4

SALT2 Light Curve Fit Parameters for ESSENCE SN Ia and "Ia?" Objects

\begin{tabular}{|c|c|c|c|c|c|c|c|c|c|c|c|c|}
\hline ID & $m_{B}{ }^{\mathrm{a}}$ & $\sigma_{m_{B}}$ & $m_{V}$ & $\sigma_{m_{V}}$ & $T_{\max }^{B} \mathrm{~b}$ & $\sigma_{T_{\max }}$ & $x_{1}$ & $\sigma_{x_{1}}$ & $c^{\mathrm{c}}$ & $\sigma_{c}$ & $\operatorname{Cov}\left(c, x_{1}\right)^{\mathrm{d}}$ & $\mathrm{Q}^{\mathrm{e}}$ \\
\hline b010 & 23.4583 & 0.0666 & 23.5138 & 0.0937 & 52593.2578 & 0.8841 & 0.9105 & 0.7618 & -0.0781 & 0.1012 & 0.0248 & $\mathrm{~T}$ \\
\hline g050 & 23.2239 & 0.0702 & 23.4278 & 0.1084 & 53302.2280 & 0.5811 & -0.2400 & 0.5682 & -0.2226 & 0.1069 & 0.0153 & $\mathrm{~T}$ \\
\hline h323 & 23.4921 & 0.0594 & 23.3174 & 0.0873 & 53329.8365 & 0.6337 & 0.6074 & 0.5493 & 0.1485 & 0.0930 & 0.0094 & $\mathrm{~F}$ \\
\hline n322 & 24.3561 & 0.0919 & 24.4587 & 0.2019 & 53707.8798 & 1.4726 & 0.1779 & 1.0967 & -0.1237 & 0.1342 & 0.0518 & $\mathrm{~T}$ \\
\hline
\end{tabular}

Notes.

${ }^{\text {a }}$ SALT2 does not directly report distance estimates. The distance modulus is determined using a global fit for all SN Ia together with other cosmological parameters.

${ }^{\mathrm{b}}$ While the SALT2 shape estimates are strongly affected by measurements pre-maximum, it uses significantly more low S/N measurements on the decline, as well as measurements for faint and/or high- $z$ objects. Consequently, the cut on number of measurements post-maximum has very little impact. We instead require a total of eight observations be used in the fit to ensure that the measured parameters are reliable.

c We use the updated SALT2 color law described by Guy et al. (2010). This differs significantly from the O'Donnell (1994) extinction law in the near-UV.

${ }^{\mathrm{d}}$ Covariances between all the fit parameters - $\operatorname{Cov}\left(m_{B}, x_{1}\right), \operatorname{Cov}\left(m_{B}, c\right)$, and $\operatorname{Cov}\left(x_{1}, c\right)$-are calculated, and these values will be included in the machine readable tables provided with this work.

${ }^{\mathrm{e}}$ Flag describing if the object passes (T) or fails (F) the light curve quality cuts for SALT described by WV07 and the shape and color cuts described by Conley et al. (2011).

derived light curve shape and extinction are generally less susceptible to poor phase coverage.

\subsubsection{SALT2 Light Curve Analysis}

Additionally, we employ version $2.2 .0 \mathrm{~b}$ of SALT2 released together with G10. SALT2 is a flux-based fitter and employs measurements of the baseline flux to restrict the search range for fitted parameters. However, only data within the rest-frame phase range $-15<\Phi<45$ days are used in the $\chi^{2}$ minimization. Measurements in observer frame filters that map to the rest-frame wavelength range $3000<\lambda<7000 \AA$ are used in the fit. Model and $K$ correction uncertainties are propagated into the error matrix, and an additional $U$ band calibration uncertainty of $0.1 \mathrm{mag}$ is added in quadrature for low- $z$ NUV data.

The SALT2 light curve shape, $x_{1}$, and color, $c$, distributions for ESSENCE SN Ia and "Ia?" objects are shown in Figure 13. The SALT2 light curve fit parameters for the ESSENCE sample are provided in Table 4. Additionally, we have indicated whether the objects pass a combination of the light curve quality cuts used by WV07 for the four-year sample, as well as shape and color cuts employed by Conley et al. (2011) and Scolnic et al. (2014b).

Objects with $x_{1}<-3.0$ and $x_{1}>3.0$ are poorly represented in the SALT2 training sample. Fits with these values often have unconstrained rises or peaks, and provide unreliable distance estimates. 
Table 5

Summary of the Systematic Uncertainties on Calibrated CTIO Blanco Natural Magnitudes from ESSENCE

\begin{tabular}{lcc}
\hline \hline Effect & $\begin{array}{c}\Delta_{R} \\
(\mathrm{mag})\end{array}$ & $\begin{array}{c}\Delta_{I} \\
(\mathrm{mag})\end{array}$ \\
\hline Shutter precision & \multicolumn{1}{c}{ Errors in the Measurement of Flux } \\
Detector linearity & $<0.001$ & $<0.001$ \\
Image De-trending & 0.005 & 0.005 \\
Astrometric Uncertainties $\quad$ Errors in the Photometric Calibration & \pm 0.005 \\
& $\mp 0.001$ & 0.005 \\
$\pm 10 \%$ error in airmass relation & \pm 0.005 & $\mp 0.002$ \\
Uncertainties in color term & 0.003 & \pm 0.005 \\
Uncertainties in the zero point & $<0.001$ & 0.001 \\
Uncertainties in extrapolating zero points & \pm 0.002 & $<0.001$ \\
Magnitudes of BD $+17^{\circ} 4708$ & \pm 0.011 & \pm 0.002 \\
\hline Total & \pm 0.002 & \pm 0.010 \\
\hline SED of BD $+17^{\circ} 4708$ & \pm 0.012 & \pm 0.011 \\
\hline Total & & \\
\hline
\end{tabular}

Note. Italicized entries are sources of increased dispersion on distance moduli, but do not introduce systematic bias.

Conley et al. (2011) required that $-0.25<c<0.25$ mag to eliminate blue objects that were not adequately represented in the training sample, as well as objects with very red colors, which they believe are the result of a combination of different effects. It is possible that extinction in the host galaxy in the SN Ia is one of these effects. Three objects, m040, m070, and $m 075$, are catastrophic outliers and are not displayed here. All three were discovered near the start of the 2005 observing season, and none has any pre-maximum photometry.

\section{SYSTEMATICS AFFECTING THE ESSENCE SURVEY PHOTOMETRY}

Here we identify and assess the size of each effect using empirical tests of internal and absolute photometric calibration. Wherever possible, we quantify systematics by directly introducing a bias at either the image or catalog level and propagating the bias through our pipeline to measure the effect on output photometry. We also compare our photometry to SDSS photometry converted to the Landolt system to set an upper limit on our systematic error budget and evaluate our absolute photometric calibration in the different ESSENCE fields.

The systematic effects can be divided into two categories.

1. Effects that cause errors in individual photometric measurements, and correlate with distance, leading to a bias in cosmological inference.

2. Effects that cause errors in individual photometric measurements, but are not correlated with distance, and therefore do not bias the cosmological result, but nevertheless lead to increased dispersion in Hubble residuals.

We list the various sources of photometric error in Table 5, and detail and estimate the effect of each in the subsections that follow.
NARAYAN ET AL.

\subsection{Shutter Precision}

The MOSAIC II shutter is described in Section 2. The shutter blades take $23 \mathrm{~ms}$ to cover the entire field, leading to $\mathrm{a} \pm 0.5 \%$ non-uniformity for a one second exposure. This is a negligible correction for the exposure times of all ESSENCE science (200s in $R$ and $400 \mathrm{~s}$ in $I$ ) and calibration frames $(>10 \mathrm{~s}$ in both filters).

\subsection{Detector Linearity}

We imaged the Ru149 field in $R$ and $I$ varying the exposure times from 2 to $400 \mathrm{~s} .{ }^{37}$ Fluxes are measured for isolated stars using a fixed 20 pixel aperture radius and corrected for extinction. These stars span the dynamic range of the detector below saturation. We compute residuals to the average magnitude for each star, and compute the $3 \sigma$ clipped average residuals for all stars. We find that these average residuals are $<0.005$ mag over the entire range of exposure times for both filters so we infer that the detector is linear to $\sim 0.5 \%$. We also examined the difference between our measured instruemental magnitudes and catalog magnitudes, at constant exposure time, to check if there was any departure from linearity with flux. We do not see any evidence of nonlinearity with flux below saturation.

\subsection{Systematic Uncertainties with Image De-trending}

We avoid most long period systematic errors with image preprocessing by using biases and flat fields obtained nightly, rather than a global bias or flat field for a full observing season. Any systematic errors caused by a misestimation of the bias or the flat field will only affect measurements made on a single night. While photometric measurements of objects observed on those nights will be systematically biased, this error does not affect photometric measurements of the same objects from other nights. Consequently, the effect is very unlikely to correlate with distance modulus, and will not lead to a bias in cosmological measurements.

The systematic uncertainties associated with the illumination correction are estimated in Appendix A and found to be $<0.3 \%$. We typically obtain $\sim 10$ bias and dome flat images in each filter each observing night. Comparing the combined bias and flat-field images of consecutive nights, we find differences of $\sim 0.1 \%$. As these errors can occur simultaneously, and affect the image processing additively, we adopt a $0.5 \%$ error as the systematic error associated with our image de-trending.

\subsection{Astrometric Uncertainties}

The astrometric uncertainty of a single detection is composed of a systematic floor and a term that is inversely proportional to the $\mathrm{S} / \mathrm{N}$ and directly proportional to the FWHM of the detection:

$$
\sigma_{a}^{2}=\sigma_{\mathrm{sys}}^{2}+\sigma_{d}^{2}\left(\frac{\mathrm{FWHM}}{\mathrm{SNR}}\right)^{2} .
$$

We use the procedure detailed in Rest et al. (2014, Appendix A) and find that the single-epoch positions for supernovae are accurate to within 0 !" 02 . M07 measured the impact of such an offset by identifying sources of known flux with FWHM typical for the survey, and measuring their flux through a PSF

\footnotetext{
$\overline{37}$ Exposures under $10 \mathrm{~s}$ are not used outside this analysis of detector linearity.
} 
Table 6

Transient Objects Considered for Follow-up Observations by the ESSENCE Survey

\begin{tabular}{|c|c|c|c|c|c|c|c|c|c|c|c|}
\hline ESSENCE ID & IAU ID & Type & Subfield & Amp & R.A. (J2000) & decl. & $z_{\mathrm{SNID}}$ & $\sigma_{z}$ & $z_{\mathrm{GAL}}$ & $\sigma_{z_{\mathrm{GAL}}}$ & $\mathrm{MW}_{E(B-V)}$ \\
\hline $\mathrm{a} 002$ & $\ldots$ & Gal & wxc1 & 4 & $23: 34: 48.580$ & $-10: 11: 02.260$ & $\ldots$ & $\ldots$ & 0.3155 & 0.0002 & 0.02469 \\
\hline b001 & $\ldots$ & Unk & wxc1 & 14 & 23:36:07.860 & $-10: 02: 26.660$ & $\ldots$ & $\ldots$ & $\ldots$ & $\ldots$ & 0.02580 \\
\hline b002 & $\ldots$ & Star & wxh1 & 1 & $00: 12: 51.140$ & $-10: 38: 27.050$ & $\ldots$ & $\ldots$ & -0.0003 & 0.0002 & 0.03826 \\
\hline b003 & $2002 \mathrm{iu}$ & Ia & wx06 & 14 & $00: 13: 33.100$ & $-10: 13: 09.920$ & 0.115 & 0.005 & $\ldots$ & $\ldots$ & 0.03980 \\
\hline b004 & $2002 \mathrm{iv}$ & $\mathrm{IaT}$ & wx17 & 6 & 02:19:16.110 & $-07: 44: 06.720$ & 0.226 & 0.006 & 0.2283 & 0.0006 & 0.02329 \\
\hline b005 & 2002iw & Gal & wxd1 & 11 & 23:43:07.250 & $-09: 48: 05.200$ & $\ldots$ & $\ldots$ & 0.2048 & 0.0003 & 0.02989 \\
\hline b006 & $2002 \mathrm{ix}$ & II? & waa7 & 16 & $23: 31: 00.863$ & $-09: 29: 07.205$ & $\ldots$ & $\ldots$ & $\ldots$ & $\ldots$ & 0.02854 \\
\hline b008 & $2002 j q$ & Ia & wx01 & 5 & $23: 35: 57.960$ & $-10: 05: 56.880$ & 0.477 & 0.005 & $\ldots$ & $\ldots$ & 0.02647 \\
\hline b010 & 2002iy & Ia & wdd5 & 7 & 02:30:40.022 & $-08: 11: 40.469$ & 0.594 & 0.005 & 0.587 & 0.0010 & 0.03252 \\
\hline b013 & $2002 \mathrm{iz}$ & Ia & wdd5 & 10 & 02:31:20.745 & $-08: 36: 13.414$ & 0.427 & 0.003 & 0.4274 & 0.0005 & 0.03283 \\
\hline b014 & $\ldots$ & Gal & wdd5 & 15 & 02:31:11.408 & $-08: 10: 53.113$ & $\ldots$ & $\ldots$ & 0.2689 & 0.0002 & 0.03390 \\
\hline b015 & $\ldots$ & Gal & wcx1 & 9 & $23: 36: 44.070$ & $-10: 24: 23.300$ & $\ldots$ & $\ldots$ & 0.2070 & 0.0002 & 0.02875 \\
\hline b016 & $2002 \mathrm{ja}$ & Ia & waa7 & 15 & 23:30:09.685 & $-09: 35: 01.809$ & 0.334 & 0.008 & $\ldots$ & $\ldots$ & 0.03111 \\
\hline b017 & $2002 \mathrm{jb}$ & Ia & waa7 & 6 & 23:29:44.149 & $-09: 36: 34.508$ & 0.272 & 0.006 & $\ldots$ & $\ldots$ & 0.03442 \\
\hline b019 & $\ldots$ & Gal & wxd1 & 4 & $23: 40: 47.490$ & $-09: 42: 19.340$ & $\ldots$ & $\ldots$ & 0.2129 & 0.0007 & 0.03084 \\
\hline b020 & 2002jr & Ia & wec 9 & 1 & 02:04:41.039 & $-05: 09: 40.727$ & 0.431 & 0.004 & $\ldots$ & $\ldots$ & 0.02485 \\
\hline b022 & $2002 \mathrm{jc}$ & Ia & wec 2 & 3 & $02: 07: 27.285$ & $-03: 50: 20.744$ & 0.534 & 0.008 & $\ldots$ & $\ldots$ & 0.02457 \\
\hline b023 & $2002 \mathrm{js}$ & Ia & wx 18 & 9 & $02: 20: 35.390$ & $-09: 34: 43.900$ & 0.557 & 0.003 & $\ldots$ & $\ldots$ & 0.02399 \\
\hline b024 & $\ldots$ & Star & wxc1 & 16 & $23: 36: 31.330$ & $-09: 55: 01.600$ & $\ldots$ & $\ldots$ & 0.0001 & 0.0002 & 0.02861 \\
\hline b025 & $\ldots$ & Unk & wxal & 5 & $23: 24: 11.150$ & $-09: 13: 20.120$ & $\ldots$ & $\ldots$ & $\ldots$ & $\ldots$ & 0.03153 \\
\hline b027 & $2002 \mathrm{jd}$ & Ia & wx11 & 16 & $00: 28: 38.390$ & $+00: 40: 29.290$ & 0.316 & 0.003 & $\ldots$ & $\ldots$ & 0.02587 \\
\hline $\mathrm{c002}$ & $\ldots$ & Unk & wbb6 & 14 & 01:10:05.029 & $+00: 16: 31.437$ & $\ldots$ & $\ldots$ & $\ldots$ & $\ldots$ & 0.02483 \\
\hline $\mathrm{c003}$ & $2002 \mathrm{jt}$ & Ia & wx06 & 15 & $00: 13: 36.700$ & $-10: 08: 24.000$ & 0.565 & 0.006 & $\ldots$ & $\ldots$ & 0.03682 \\
\hline $\mathrm{c} 005$ & $\ldots$ & AGN & waa7 & 10 & $23: 30: 54.777$ & $-09: 56: 47.863$ & 0.248 & 0.002 & $\ldots$ & $\ldots$ & 0.02350 \\
\hline $\mathrm{c} 012$ & $2002 \mathrm{ju}$ & Ia & wx18 & 16 & 02:20:11.000 & $-09: 04: 37.500$ & 0.349 & 0.005 & 0.3473 & 0.0001 & 0.02649 \\
\hline c014 & $2002 \mathrm{jv}$ & $\mathrm{Ib}$ & wcc3 & 3 & 02:04:34.815 & $-03: 51: 57.919$ & $\ldots$ & $\ldots$ & 0.2203 & 0.0001 & 0.02400 \\
\hline $\mathrm{c} 015$ & $2002 \mathrm{jw}$ & Ia & wdd5 & 2 & 02:30:00.539 & $-08: 36: 22.561$ & 0.356 & 0.007 & 0.3575 & 0.0006 & 0.03399 \\
\hline c016 & $2002 j x$ & Gal & wxm1 & 4 & $00: 26: 54.136$ & $+00: 22: 49.750$ & $\ldots$ & $\ldots$ & 0.8446 & 0.0004 & 0.02350 \\
\hline $\mathrm{c020}$ & $\ldots$ & Unk & wxt2 & 15 & $02: 20: 32.124$ & $-07: 36: 02.690$ & $\ldots$ & $\ldots$ & $\ldots$ & $\ldots$ & 0.02342 \\
\hline $\mathrm{c} 022$ & $\ldots$ & $\mathrm{Ib}$ & wxu2 & 15 & 02:21:06.336 & $-09: 06: 51.820$ & $\ldots$ & $\ldots$ & 0.2123 & 0.0003 & 0.02529 \\
\hline $\mathrm{c} 023$ & $\ldots$ & Ia & wx11 & 15 & 00:28:03.160 & $+00: 37: 50.430$ & 0.412 & 0.011 & 0.3987 & 0.0003 & 0.02295 \\
\hline $\mathrm{c} 024$ & $\ldots$ & Gal & wdd5 & 5 & 02:29:56.521 & $-08: 23: 52.283$ & $\ldots$ & $\ldots$ & 0.3174 & 0.0001 & 0.03786 \\
\hline $\mathrm{c} 025$ & $\ldots$ & AGN & waa7 & 14 & 23:30:18.309 & $-09: 38: 02.438$ & 0.361 & 0.002 & $\ldots$ & $\ldots$ & 0.02845 \\
\hline $\mathrm{c027}$ & $\ldots$ & Gal & wxm1 & 4 & $00: 27: 56.787$ & $+00: 25: 19.760$ & $\ldots$ & $\ldots$ & 0.4534 & 0.0002 & 0.02240 \\
\hline $\mathrm{c} 028$ & $\ldots$ & AGN & wxu2 & 16 & 02:20:03.304 & $-09: 05: 20.990$ & 2.033 & 0.004 & $\ldots$ & $\ldots$ & 0.02664 \\
\hline d009 & $\ldots$ & Ia & waa6 & 16 & $23: 25: 55.900$ & $-08: 56: 41.300$ & 0.351 & 0.002 & 0.3535 & 0.0002 & 0.03752 \\
\hline d010 & 2003jp & Ic & waa6 & 16 & $23: 26: 03.281$ & $-08: 59: 22.829$ & $\ldots$ & $\ldots$ & 0.0829 & 0.0001 & 0.03668 \\
\hline d029 & $\ldots$ & AGN & waa6 & 13 & $23: 25: 11.206$ & $-09: 13: 38.505$ & 2.584 & 0.003 & $\ldots$ & $\ldots$ & 0.03429 \\
\hline d033 & 2003jo & Ia & waa6 & 10 & $23: 25: 24.047$ & $-09: 26: 00.659$ & 0.530 & 0.008 & 0.5251 & 0.0003 & 0.03621 \\
\hline d034 & $\ldots$ & AGN & waa7 & 10 & $23: 30: 31.616$ & $-09: 56: 24.390$ & 2.285 & 0.007 & $\ldots$ & $\ldots$ & 0.02421 \\
\hline d051 & $\ldots$ & Gal & wec8 & 2 & 02:06:48.703 & $-05: 08: 46.023$ & $\ldots$ & $\ldots$ & 0.3817 & 0.0002 & 0.02180 \\
\hline d057 & $2003 \mathrm{jk}$ & Unk & wbb6 & 3 & 01:08:06.169 & $+00: 02: 20.636$ & $\ldots$ & $\ldots$ & $\ldots$ & $\ldots$ & 0.02948 \\
\hline d058 & $2003 \mathrm{jj}$ & Ia & wbb6 & 3 & 01:07:58.519 & $+00: 03: 01.918$ & 0.589 & 0.009 & 0.5839 & 0.0002 & 0.02883 \\
\hline d059 & $\ldots$ & Gal & wec5 & 3 & 02:06:49.459 & $-04: 26: 47.244$ & $\ldots$ & $\ldots$ & 0.2076 & 0.0002 & 0.02262 \\
\hline d060 & $\ldots$ & Star & wec7 & 3 & 02:09:02.757 & $-05: 03: 39.667$ & 0.001 & 0.003 & $\ldots$ & $\ldots$ & 0.02159 \\
\hline d062 & $\ldots$ & AGN & wec 9 & 3 & 02:04:19.323 & $-05: 01: 44.661$ & 2.433 & 0.004 & $\ldots$ & $\ldots$ & 0.02430 \\
\hline d083 & 2003jn & IaT & wdd9 & 12 & 02:29:21.199 & $-09: 02: 15.490$ & 0.330 & 0.006 & $\ldots$ & $\ldots$ & 0.02798 \\
\hline d084 & 2003jm & Ia & wdd9 & 11 & 02:28:50.940 & $-09: 09: 58.077$ & 0.516 & 0.006 & 0.5221 & 0.0002 & 0.02516 \\
\hline d085 & 2003jv & Ia & waa5 & 16 & $23: 27: 58.197$ & $-08: 57: 11.687$ & 0.401 & 0.008 & 0.4047 & 0.0001 & 0.03495 \\
\hline d086 & $2003 j u$ & Ia & waa5 & 3 & $23: 27: 01.704$ & $-09: 24: 04.573$ & 0.201 & 0.003 & $\ldots$ & $\ldots$ & 0.03459 \\
\hline d087 & 2003jr & Ia & wbb5 & 4 & 01:11:06.232 & $+00: 13: 44.210$ & 0.337 & 0.004 & 0.3400 & 0.0003 & 0.02516 \\
\hline d089 & 2003jl & Ia & wdd6 & 8 & $02: 28: 28.568$ & $-08: 08: 44.932$ & 0.425 & 0.005 & $\ldots$ & $\ldots$ & 0.02997 \\
\hline d091 & $\ldots$ & Unk & wcc1 & 2 & 02:09:35.211 & $-03: 56: 17.327$ & $\ldots$ & $\ldots$ & $\ldots$ & $\ldots$ & 0.02298 \\
\hline d093 & $2003 \mathrm{js}$ & Ia & wdd5 & 3 & 02:29:52.152 & $-08: 32: 28.155$ & 0.361 & 0.003 & 0.3636 & 0.0001 & 0.03212 \\
\hline d097 & 2003jt & Ia & wdd5 & 10 & 02:31:54.595 & $-08: 35: 48.609$ & 0.430 & 0.005 & $\ldots$ & $\ldots$ & 0.03114 \\
\hline d099 & 2003ji & Ia & wcc2 & 16 & 02:07:54.841 & $-03: 28: 28.055$ & 0.216 & 0.003 & $\ldots$ & $\ldots$ & 0.02456 \\
\hline d 100 & 2003jq & $\mathrm{IaP}$ & waa7 & 16 & 23:30:51.191 & $-09: 28: 34.044$ & 0.158 & 0.003 & $\ldots$ & $\ldots$ & 0.02863 \\
\hline d 115 & $\ldots$ & Unk & wbb6 & 11 & 01:09:45.163 & $+00: 02: 02.740$ & $\ldots$ & $\ldots$ & $\ldots$ & $\ldots$ & 0.03106 \\
\hline d117 & 2003jw & Ia & wdd 8 & 16 & 02:31:06.836 & $-08: 45: 36.535$ & 0.301 & 0.005 & 0.2968 & 0.0002 & 0.02918 \\
\hline d120 & $\ldots$ & AGN & wcc1 & 2 & 02:09:44.494 & $-03: 57: 02.923$ & 1.279 & 0.005 & $\ldots$ & $\ldots$ & 0.02299 \\
\hline d 123 & $\ldots$ & Gal & wec 9 & 16 & 02:06:08.565 & $-04: 39: 08.710$ & $\ldots$ & $\ldots$ & 0.4995 & 0.0004 & 0.02430 \\
\hline d124 & $\ldots$ & AGN & wec 9 & 15 & 02:06:04.554 & $-04: 41: 45.145$ & 0.617 & 0.023 & $\ldots$ & $\ldots$ & 0.02348 \\
\hline d149 & 2003jy & Ia & wec4 & 11 & 02:10:53.987 & $-04: 25: 49.436$ & 0.344 & 0.008 & 0.3388 & 0.0002 & 0.02020 \\
\hline $\mathrm{d} 150$ & $\ldots$ & Gal & wcc1 & 12 & $02: 10: 12.486$ & $-03: 49: 09.928$ & $\ldots$ & $\ldots$ & 0.1910 & 0.0003 & 0.02450 \\
\hline
\end{tabular}


Table 6

(Continued)

\begin{tabular}{|c|c|c|c|c|c|c|c|c|c|c|c|}
\hline ESSENCE ID & IAU ID & Type & Subfield & Amp & R.A. (J2000) & decl. & $z_{\mathrm{SNID}}$ & $\sigma_{z}$ & $z_{\mathrm{GAL}}$ & $\sigma_{z_{\mathrm{GAL}}}$ & $\mathrm{MW}_{E(B-V)}$ \\
\hline d156 & $2003 j x$ & Unk & wcc2 2 & 4 & 02:06:33.398 & $-03: 48: 39.117$ & $\ldots$ & $\ldots$ & $\ldots$ & $\ldots$ & 0.02311 \\
\hline e018 & $\ldots$ & AGN & wbb7 & 2 & $01: 13: 59.547$ & $+00: 32: 48.750$ & 0.181 & 0.001 & $\ldots$ & $\ldots$ & 0.02879 \\
\hline $\mathrm{e} 020$ & $2003 \mathrm{kk}$ & Ia & waa6 & 9 & $23: 25: 36.054$ & $-09: 31: 44.807$ & 0.159 & 0.006 & 0.1643 & 0.0008 & 0.03411 \\
\hline $\mathrm{e} 022$ & $2003 \mathrm{kj}$ & IIP & wbb7 & 12 & $01: 14: 36.556$ & $+00: 23: 58.180$ & $\ldots$ & $\ldots$ & 0.0784 & 0.0001 & 0.02926 \\
\hline $\mathrm{e} 025$ & $\ldots$ & Gal & wdd3 & 15 & 02:29:07.399 & $-07: 36: 34.479$ & $\ldots$ & $\ldots$ & 0.1797 & 0.0002 & 0.02982 \\
\hline $\mathrm{e} 027$ & $\ldots$ & Gal & wec7 & 16 & 02:11:09.129 & $-04: 39: 19.417$ & $\ldots$ & $\ldots$ & 0.8043 & 0.0000 & 0.01969 \\
\hline e029 & $2003 \mathrm{kl}$ & $\mathrm{Ia}$ & wbb3 & 15 & 01:09:48.798 & $+01: 00: 05.496$ & 0.332 & 0.008 & 0.3333 & 0.0009 & 0.03509 \\
\hline e103 & $\ldots$ & Unk & wbb9 & 2 & 01:09:32.340 & $+00: 36: 43.930$ & $\ldots$ & $\ldots$ & $\ldots$ & $\ldots$ & 0.02437 \\
\hline e106 & $\ldots$ & Unk & wbb6 & 11 & 01:09:45.163 & $+00: 02: 02.740$ & $\ldots$ & $\ldots$ & 0.3219 & 0.0009 & 0.03106 \\
\hline e108 & $2003 \mathrm{~km}$ & Ia & wdd8 & 4 & 02:30:09.001 & $-09: 04: 35.621$ & 0.473 & 0.009 & $\ldots$ & $\ldots$ & 0.03216 \\
\hline e118 & $\ldots$ & AGN & waa5 & 11 & $23: 27: 48.448$ & $-09: 22: 53.295$ & 0.552 & 0.007 & $\ldots$ & $\ldots$ & 0.03665 \\
\hline e119 & $\ldots$ & Gal & wbb1 & 7 & $01: 14: 16.912$ & $+01: 03: 06.427$ & $\ldots$ & $\ldots$ & 0.5584 & 0.0002 & 0.03121 \\
\hline e120 & $\ldots$ & Gal & waa5 & 9 & $23: 28: 37.492$ & $-09: 30: 30.569$ & $\ldots$ & $\ldots$ & 0.2974 & 0.0002 & 0.03937 \\
\hline e132 & $2003 \mathrm{kn}$ & $\mathrm{Ia}$ & wcc1 & 7 & 02:09:15.549 & $-03: 35: 41.010$ & 0.235 & 0.006 & 0.2443 & 0.0003 & 0.02585 \\
\hline e133 & $\ldots$ & Gal & wcc1 & 7 & 02:09:17.662 & $-03: 35: 41.255$ & $\ldots$ & $\ldots$ & 0.2450 & 0.0003 & 0.02596 \\
\hline e136 & $2003 \mathrm{ko}$ & $\mathrm{Ia}$ & wcc1 & 12 & 02:11:06.495 & $-03: 47: 55.899$ & 0.348 & 0.006 & 0.3602 & 0.0008 & 0.02329 \\
\hline e138 & $2003 \mathrm{kt}$ & Ia & wdd4 & 1 & $02: 33: 46.992$ & $-08: 36: 22.141$ & 0.608 & 0.006 & $\ldots$ & $\ldots$ & 0.03294 \\
\hline e140 & $2003 \mathrm{kq}$ & $\mathrm{IaT}$ & wdd5 & 15 & 02:31:04.089 & $-08: 10: 56.603$ & 0.614 & 0.006 & 0.6060 & 0.0002 & 0.03341 \\
\hline e141 & $\ldots$ & $\mathrm{Ib}$ & wdd7 & 2 & $02: 32: 30.272$ & $-09: 05: 53.662$ & $\ldots$ & $\ldots$ & 0.0982 & 0.0001 & 0.02972 \\
\hline e143 & $\ldots$ & Unk & wdd7 & 3 & 02:33:11.715 & $-09: 03: 32.183$ & $\ldots$ & $\ldots$ & 0.1107 & 0.0001 & 0.02632 \\
\hline e147 & $2003 \mathrm{kp}$ & Ia & wdd5 & 9 & $02: 31: 02.652$ & $-08: 39: 50.909$ & 0.647 & 0.008 & $\ldots$ & $\ldots$ & 0.03419 \\
\hline e148 & $2003 \mathrm{kr}$ & Ia & wdd5 & 10 & $02: 31: 20.960$ & $-08: 36: 14.195$ & 0.431 & 0.006 & 0.427 & 0.0010 & 0.03281 \\
\hline e149 & $2003 \mathrm{ks}$ & Ia & wdd5 & 10 & $02: 31: 34.528$ & $-08: 36: 46.462$ & 0.498 & 0.008 & $\ldots$ & $\ldots$ & 0.03166 \\
\hline e309 & $\ldots$ & Star & waa9 & 14 & $23: 25: 14.230$ & $-09: 44: 25.810$ & 0.001 & 0.003 & $\ldots$ & $\ldots$ & 0.02968 \\
\hline e315 & $2003 \mathrm{ku}$ & Ia & wbb9 & 3 & 01:08:36.253 & $-00: 33: 20.780$ & $\ldots$ & $\ldots$ & $\ldots$ & $\ldots$ & 0.03573 \\
\hline e418 & $\ldots$ & Unk & wcc2 & 8 & 02:07:30.851 & $-03: 30: 49.497$ & $\ldots$ & $\ldots$ & $\ldots$ & $\ldots$ & 0.02304 \\
\hline e501 & $\ldots$ & Unk & waal & 1 & $23: 29: 20.159$ & $-08: 54: 27.836$ & $\ldots$ & $\ldots$ & $\ldots$ & $\ldots$ & 0.03161 \\
\hline e504 & $\ldots$ & AGN & waa3 & 4 & $23: 25: 01.338$ & $-08: 41: 49.753$ & 0.675 & 0.005 & $\ldots$ & $\ldots$ & 0.04289 \\
\hline e510 & $\ldots$ & Unk & waal & 13 & $23: 30: 59.971$ & $-08: 37: 34.344$ & $\ldots$ & $\ldots$ & $\ldots$ & $\ldots$ & 0.03116 \\
\hline e528 & $\ldots$ & Unk & wec5 & 3 & 02:07:37.767 & $-04: 27: 06.738$ & $\ldots$ & $\ldots$ & $\ldots$ & $\ldots$ & 0.02129 \\
\hline e529 & $\ldots$ & Unk & wec5 & 3 & $02: 06: 42.954$ & $-04: 26: 31.293$ & $\ldots$ & $\ldots$ & $\ldots$ & $\ldots$ & 0.02226 \\
\hline e531 & $2003 \mathrm{kv}$ & Ia? & wec 1 & 4 & 02:09:42.519 & $-03: 46: 48.442$ & $\ldots$ & $\ldots$ & $\ldots$ & $\ldots$ & 0.02288 \\
\hline e604 & $\ldots$ & Gal & waa6 & 8 & $23: 23: 51.868$ & $-08: 59: 17.456$ & $\ldots$ & $\ldots$ & 0.4357 & 0.0001 & 0.03519 \\
\hline f001 & $2003 \lg$ & IIP & wbb7 & 1 & $01: 13: 32.675$ & $+00: 36: 57.310$ & 0.171 & 0.006 & $\ldots$ & $\ldots$ & 0.02730 \\
\hline f011 & 2003lh & Ia & wcc7 & 12 & 02:10:19.505 & $-04: 59: 32.063$ & 0.544 & 0.006 & $\ldots$ & $\ldots$ & 0.02000 \\
\hline f017 & $\ldots$ & AGN & wdd 9 & 10 & $02: 28: 38.844$ & $-09: 11: 09.202$ & 0.725 & 0.004 & $\ldots$ & $\ldots$ & 0.02542 \\
\hline f041 & 2003le & Ia & wbb6 & 8 & 01:08:08.739 & $+00: 27: 09.580$ & 0.560 & 0.004 & $\ldots$ & $\ldots$ & 0.02934 \\
\hline f044 & $\ldots$ & Ia & wbb8 & 8 & 01:11:20.561 & $+00: 04: 10.020$ & $\ldots$ & $\ldots$ & 0.4078 & 0.0003 & 0.02544 \\
\hline f076 & 2003lf & $\mathrm{Ia}$ & wbb9 & 1 & 01:08:49.807 & $-00: 44: 13.490$ & 0.408 & 0.004 & $\ldots$ & $\ldots$ & 0.03948 \\
\hline f095 & $\ldots$ & Gal & wcc2 & 8 & 02:06:56.203 & $-03: 31: 07.936$ & $\ldots$ & $\ldots$ & 0.3130 & 0.0008 & 0.02336 \\
\hline f096 & $2003 \mathrm{~lm}$ & $\mathrm{Ia}$ & waa3 & 3 & $23: 24: 25.501$ & $-08: 45: 50.834$ & 0.413 & 0.004 & 0.4080 & 0.0001 & 0.04193 \\
\hline $\mathrm{f} 123$ & $\ldots$ & $\mathrm{Ia}$ & wcc1 & 7 & 02:09:57.282 & $-03: 32: 26.609$ & 0.534 & 0.009 & 0.5261 & 0.0002 & 0.02410 \\
\hline $\mathrm{f} 213$ & $\ldots$ & Unk & wbb4 & 12 & $01: 14: 50.770$ & $+00: 14: 35.919$ & $\ldots$ & $\ldots$ & $\ldots$ & $\ldots$ & 0.03231 \\
\hline $\mathrm{f} 216$ & 200311 & Ia & wdd4 & 15 & $02: 35: 41.190$ & $-08: 06: 29.788$ & 0.595 & 0.011 & 0.5958 & 0.0001 & 0.03288 \\
\hline $\mathrm{f} 221$ & $2003 \mathrm{lk}$ & Ia & wcc4 & 14 & $02: 11: 12.817$ & $-04: 13: 52.110$ & 0.443 & 0.004 & 0.4413 & 0.0003 & 0.02044 \\
\hline $\mathrm{f} 231$ & $2003 \ln$ & Ia & waal & 13 & $23: 30: 27.131$ & $-08: 35: 46.927$ & 0.615 & 0.003 & $\ldots$ & $\ldots$ & 0.02860 \\
\hline $\mathrm{f} 235$ & $20031 \mathrm{j}$ & Ia & wbb5 & 13 & $01: 12: 10.034$ & $+00: 19: 51.267$ & 0.422 & 0.006 & 0.4171 & 0.0006 & 0.03243 \\
\hline $\mathrm{f} 244$ & 2003li & Ia & wdd3 & 8 & $02: 27: 47.294$ & $-07: 33: 46.220$ & 0.546 & 0.005 & 0.5403 & 0.0002 & 0.02690 \\
\hline $\mathrm{f} 247$ & $\ldots$ & Gal & wbb8 & 10 & $01: 12: 32.219$ & $+00: 31: 12.730$ & $\ldots$ & $\ldots$ & 0.4306 & 0.0003 & 0.03018 \\
\hline $\mathrm{f} 301$ & $\ldots$ & Ia & wdd6 & 1 & $02: 27: 26.513$ & $-08: 42: 24.782$ & 0.514 & 0.011 & $\ldots$ & $\ldots$ & 0.03007 \\
\hline $\mathrm{f} 304$ & $\ldots$ & Unk & wdd6 & 2 & 02:28:23.108 & $-08: 34: 22.780$ & $\ldots$ & $\ldots$ & $\ldots$ & $\ldots$ & 0.03088 \\
\hline f308 & $\ldots$ & Ia & wdd6 & 10 & 02:29:22.391 & $-08: 37: 38.480$ & 0.388 & 0.010 & $\ldots$ & $\ldots$ & 0.02955 \\
\hline $\mathrm{f} 401$ & $\ldots$ & Gal & waal & 1 & $23: 29: 40.692$ & $-08: 56: 37.030$ & $\ldots$ & $\ldots$ & 0.2023 & 0.0002 & 0.02929 \\
\hline $\mathrm{f} 441$ & $\ldots$ & Unk & wbb6 & 7 & 01:08:58.453 & $+00: 22: 15.570$ & $\ldots$ & $\ldots$ & $\ldots$ & $\ldots$ & 0.02517 \\
\hline g001 & 2004fi & Ia & waal & 1 & $23: 29: 45.348$ & $-08: 54: 36.347$ & 0.268 & 0.002 & 0.2648 & 0.0002 & 0.02895 \\
\hline g004 & $\ldots$ & Ic & wbb4 & 14 & 01:15:06.214 & $+00: 23: 38.571$ & 0.143 & 0.006 & $\ldots$ & $\ldots$ & 0.02853 \\
\hline g005 & $2004 \mathrm{fh}$ & Ia & waa2 & 13 & $23: 28: 27.197$ & $-08: 36: 55.071$ & 0.220 & 0.006 & $\ldots$ & $\ldots$ & 0.02759 \\
\hline g009 & $\ldots$ & Gal & wbb4 & 13 & $01: 14: 28.998$ & $+00: 16: 56.247$ & $\ldots$ & $\ldots$ & 0.1831 & 0.0003 & 0.02991 \\
\hline g014 & $\ldots$ & Gal & wbb1 & 6 & $01: 13: 18.202$ & $+00: 57: 00.728$ & $\ldots$ & $\ldots$ & 0.1949 & 0.0003 & 0.02787 \\
\hline g043 & $2004 \mathrm{fj}$ & IIP & wbb6 & 16 & 01:09:51.075 & $+00: 27: 20.934$ & 0.190 & 0.002 & 0.1874 & 0.0008 & 0.02529 \\
\hline g046 & $\ldots$ & Gal & wec9 & 14 & 02:05:34.300 & $-04: 46: 30.968$ & $\ldots$ & $\ldots$ & 0.1833 & 0.0002 & 0.02507 \\
\hline g050 & $2004 \mathrm{fn}$ & $\mathrm{Ia}$ & waa7 & 10 & $23: 30: 20.114$ & $-09: 58: 30.698$ & 0.616 & 0.008 & 0.6045 & 0.0003 & 0.02444 \\
\hline g052 & $2004 \mathrm{fm}$ & $\mathrm{Ia}$ & waa8 & 7 & $23: 26: 58.138$ & $-09: 37: 19.346$ & 0.381 & 0.007 & $\ldots$ & $\ldots$ & 0.03110 \\
\hline g053 & $2004 f l$ & Ia? & waa8 & 7 & $23: 26: 57.910$ & $-09: 37: 18.984$ & $\ldots$ & $\ldots$ & 0.6329 & 0.0022 & 0.03110 \\
\hline
\end{tabular}


Table 6

(Continued)

\begin{tabular}{|c|c|c|c|c|c|c|c|c|c|c|c|}
\hline ESSENCE ID & IAU ID & Type & Subfield & Amp & R.A. (J2000) & decl. & $z_{\mathrm{SNID}}$ & $\sigma_{z}$ & $z_{\mathrm{GAL}}$ & $\sigma_{z_{\mathrm{GAL}}}$ & $\mathrm{MW}_{E(B-V)}$ \\
\hline g055 & $2004 \mathrm{fk}$ & Ia & wbb7 & 7 & $01: 13: 35.842$ & $-00: 09: 27.500$ & 0.302 & 0.006 & 0.2964 & 0.0004 & 0.02823 \\
\hline g097 & $\ldots$ & Ia & waa8 & 16 & $23: 27: 37.164$ & $-09: 35: 21.041$ & 0.339 & 0.004 & 0.3434 & 0.0002 & 0.03130 \\
\hline g108 & $2004 f p$ & IIP & wdd8 & 4 & 02:29:53.012 & $-09: 01: 16.554$ & 0.162 & 0.004 & $\ldots$ & $\ldots$ & 0.03378 \\
\hline g120 & 2004 fo & Ia & wbb1 & 1 & $01: 13: 28.975$ & $+00: 35: 16.179$ & 0.507 & 0.004 & $\ldots$ & $\ldots$ & 0.02854 \\
\hline g128 & $\ldots$ & II? & waa2 & 5 & $23: 26: 43.669$ & $-08: 37: 31.458$ & $\ldots$ & $\ldots$ & 0.1642 & 0.0006 & 0.02539 \\
\hline g133 & $\ldots$ & $\mathrm{Ia}$ & wcc4 & 7 & 02:09:49.626 & $-04: 10: 55.064$ & 0.422 & 0.003 & $\ldots$ & $\ldots$ & 0.02434 \\
\hline g142 & $\ldots$ & Ia & waa2 & 11 & $23: 28: 37.713$ & $-08: 45: 03.948$ & 0.398 & 0.013 & 0.4033 & 0.0001 & 0.02842 \\
\hline g151 & $2004 \mathrm{fq}$ & Ic & waa2 & 14 & $23: 27: 45.638$ & $-08: 31: 12.785$ & 0.455 & 0.003 & 0.1462 & 0.0004 & 0.02689 \\
\hline g160 & $2004 \mathrm{fs}$ & $\mathrm{Ia}$ & wdd8 & 15 & $02: 31: 19.943$ & $-08: 49: 21.751$ & 0.507 & 0.019 & $\ldots$ & $\ldots$ & 0.02952 \\
\hline g166 & $2004 \mathrm{fr}$ & Gal & wdd9 & 14 & $02: 28: 43.772$ & $-08: 54: 24.030$ & $\ldots$ & $\ldots$ & 0.2016 & 0.0007 & 0.02988 \\
\hline g181 & $\ldots$ & Unk & wdd 9 & 2 & $02: 28: 35.984$ & $-09: 13: 43.261$ & $\ldots$ & $\ldots$ & 0.5324 & 0.0002 & 0.02629 \\
\hline g185 & $\ldots$ & II & waa2 & 1 & $23: 26: 56.854$ & $-08: 55: 12.970$ & 0.345 & 0.007 & $\ldots$ & $\ldots$ & 0.03440 \\
\hline g199 & $2004 \mathrm{ft}$ & Gal & wdd4 & 7 & $02: 33: 32.622$ & $-08: 09: 34.178$ & $\ldots$ & $\ldots$ & 0.7665 & 0.0002 & 0.03713 \\
\hline g204 & $\ldots$ & Unk & wcc2 & 13 & 02:08:26.690 & $-03: 44: 44.620$ & $\ldots$ & $\ldots$ & 0.1114 & 0.0001 & 0.02356 \\
\hline $\mathrm{g} 213$ & $\ldots$ & Gal & wbb8 & 6 & $01: 11: 54.176$ & $-00: 13: 45.690$ & $\ldots$ & $\ldots$ & 0.8423 & 0.0002 & 0.02886 \\
\hline g219 & $\ldots$ & II? & wbb9 & 10 & $01: 10: 27.165$ & $-00: 39: 11.615$ & $\ldots$ & $\ldots$ & $\ldots$ & $\ldots$ & 0.03111 \\
\hline g225 & $\ldots$ & $\mathrm{Ia}$ & waa5 & 2 & $23: 27: 15.685$ & $-09: 27: 59.728$ & 0.579 & 0.009 & $\ldots$ & $\ldots$ & 0.03504 \\
\hline g230 & $\ldots$ & Ia & wbb5 & 3 & $01: 11: 56.314$ & $+00: 07: 27.441$ & $\ldots$ & $\ldots$ & 0.3934 & 0.0001 & 0.03077 \\
\hline g240 & $\ldots$ & $\mathrm{Ia}$ & waal & 14 & $23: 30: 41.823$ & $-08: 34: 10.893$ & 0.696 & 0.007 & $\ldots$ & $\ldots$ & 0.02974 \\
\hline g276 & $\ldots$ & Gal & wcc1 & 7 & 02:09:17.715 & $-03: 35: 43.709$ & $\ldots$ & $\ldots$ & 0.2442 & 0.0002 & 0.02597 \\
\hline h280 & $\ldots$ & II & wbb6 & 10 & 01:09:28.330 & $-00: 01: 22.880$ & $\ldots$ & $\ldots$ & 0.2633 & 0.0005 & 0.03395 \\
\hline h283 & 2004ha & Ia & wec9 & 5 & 02:04:27.005 & $-04: 52: 46.192$ & 0.498 & 0.008 & $\ldots$ & $\ldots$ & 0.02678 \\
\hline h293 & $\ldots$ & Unk & wec9 & 2 & 02:05:11.580 & $-05: 09: 04.692$ & $\ldots$ & $\ldots$ & 0.5462 & 0.0001 & 0.02479 \\
\hline h296 & $\ldots$ & Gal & wdd6 & 12 & $02: 28: 45.533$ & $-08: 27: 36.835$ & $\ldots$ & $\ldots$ & 0.0590 & 0.0003 & 0.03198 \\
\hline h299 & $2004 \mathrm{hb}$ & Gal & wcc8 & 15 & 02:08:09.708 & $-04: 41: 51.880$ & $\ldots$ & $\ldots$ & 0.7186 & 0.0002 & 0.02098 \\
\hline h300 & $\ldots$ & Ia & wdd8 & 15 & $02: 31: 40.680$ & $-08: 49: 03.377$ & 0.657 & 0.003 & $\ldots$ & $\ldots$ & 0.02939 \\
\hline h304 & $\ldots$ & Gal & wcc1 & 2 & 02:09:10.894 & $-03: 58: 00.789$ & $\ldots$ & $\ldots$ & $\ldots$ & $\ldots$ & 0.02352 \\
\hline h311 & $2004 \mathrm{hc}$ & Ia & waa3 & 4 & $23: 24: 32.664$ & $-08: 41: 03.574$ & 0.752 & 0.003 & $\ldots$ & $\ldots$ & 0.04188 \\
\hline h317 & $\ldots$ & Gal & wcc8 & 10 & 02:08:21.585 & $-05: 05: 09.208$ & $\ldots$ & $\ldots$ & 0.6377 & 0.0001 & 0.02130 \\
\hline h319 & 2004hd & Ia & wec5 & 11 & 02:08:48.217 & $-04: 26: 10.319$ & 0.478 & 0.002 & 0.4903 & 0.0002 & 0.02047 \\
\hline h323 & 2004he & Ia & wdd6 & 13 & 02:29:48.797 & $-08: 20: 45.875$ & 0.603 & 0.007 & 0.5978 & 0.0003 & 0.03481 \\
\hline h336 & $\ldots$ & Gal & waa3 & 7 & $23: 24: 56.441$ & $-08: 28: 40.498$ & $\ldots$ & $\ldots$ & 0.3916 & 0.0005 & 0.03609 \\
\hline h342 & $2004 \mathrm{hf}$ & $\mathrm{Ia}$ & wdd5 & 9 & $02: 32: 00.143$ & $-08: 42: 23.852$ & 0.421 & 0.005 & $\ldots$ & $\ldots$ & 0.02867 \\
\hline h345 & 2004hg & Unk & wdd4 & 10 & $02: 34: 55.193$ & $-08: 30: 43.591$ & $\ldots$ & $\ldots$ & $\ldots$ & $\ldots$ & 0.03145 \\
\hline h352 & $\ldots$ & Gal & wcc4 & 13 & 02:10:48.002 & $-04: 17: 54.131$ & $\ldots$ & $\ldots$ & 0.1807 & 0.0002 & 0.02172 \\
\hline h353 & $\ldots$ & Gal & waa2 & 15 & $23: 28: 14.068$ & $-08: 26: 54.628$ & $\ldots$ & $\ldots$ & 0.2196 & 0.0002 & 0.02513 \\
\hline h359 & 2004hi & Ia & wec8 & 10 & 02:08:38.835 & $-05: 08: 11.825$ & 0.347 & 0.005 & $\ldots$ & $\ldots$ & 0.02095 \\
\hline h361 & $\ldots$ & Unk & wcc7 & 13 & $02: 11: 14.030$ & $-04: 53: 40.148$ & $\ldots$ & $\ldots$ & $\ldots$ & $\ldots$ & 0.02149 \\
\hline h363 & 2004hh & Ia & wec 9 & 16 & 02:06:25.028 & $-04: 38: 04.035$ & 0.211 & 0.006 & $\ldots$ & $\ldots$ & 0.02475 \\
\hline h364 & 2004hj & Ia & wdd 9 & 16 & 02:29:41.943 & $-08: 43: 49.480$ & 0.344 & 0.003 & $\ldots$ & $\ldots$ & 0.03400 \\
\hline k374 & $\ldots$ & Gal & wdd 9 & 1 & $02: 27: 34.292$ & $-09: 17: 08.085$ & $\ldots$ & $\ldots$ & 0.1423 & 0.0004 & 0.02785 \\
\hline k396 & 2004hk & Ia? & waa2 & 5 & $23: 27: 04.384$ & $-08: 38: 45.178$ & 0.271 & 0.006 & $\ldots$ & $\ldots$ & 0.02637 \\
\hline k397 & $\ldots$ & Unk & wcc1 & 7 & 02:09:31.278 & $-03: 34: 21.189$ & $\ldots$ & $\ldots$ & $\ldots$ & $\ldots$ & 0.02564 \\
\hline k402 & $\ldots$ & Unk & wbb5 & 12 & $01: 12: 54.197$ & $+00: 11: 25.151$ & $\ldots$ & $\ldots$ & $\ldots$ & $\ldots$ & 0.03139 \\
\hline k411 & $\ldots$ & $\mathrm{IaP}$ & waa3 & 10 & $23: 26: 11.781$ & $-08: 50: 17.355$ & 0.562 & 0.004 & $\ldots$ & $\ldots$ & 0.03456 \\
\hline $\mathrm{k} 425$ & $2004 \mathrm{hl}$ & Ia & wbb7 & 3 & $01: 13: 38.174$ & $-00: 27: 39.045$ & 0.274 & 0.003 & 0.2702 & 0.0001 & 0.02922 \\
\hline k426 & $\ldots$ & Gal & wdd8 & 4 & 02:30:51.203 & $-09: 04: 27.406$ & $\ldots$ & $\ldots$ & 0.7572 & 0.0003 & 0.02568 \\
\hline k429 & $2004 \mathrm{hm}$ & Ia & wdd3 & 6 & 02:28:03.110 & $-07: 42: 29.656$ & 0.171 & 0.006 & 0.1720 & 0.0006 & 0.03060 \\
\hline $\mathrm{k} 430$ & 2004hn & Ia & wbb1 & 2 & $01: 13: 32.382$ & $+00: 37: 15.455$ & 0.576 & 0.007 & $\ldots$ & $\ldots$ & 0.02719 \\
\hline k432 & $\ldots$ & Ia & waa2 & 3 & $23: 26: 46.120$ & $-08: 45: 42.405$ & 0.706 & 0.010 & $\ldots$ & $\ldots$ & 0.02786 \\
\hline k437 & 2004ho & Gal & wcc3 & 11 & 02:06:16.041 & $-03: 52: 27.803$ & $\ldots$ & $\ldots$ & 0.2878 & 0.0001 & 0.02292 \\
\hline k440 & $\ldots$ & Unk & wbb4 & 7 & $01: 14: 18.151$ & $+00: 29: 02.397$ & $\ldots$ & $\ldots$ & $\ldots$ & $\ldots$ & 0.02874 \\
\hline k441 & 2004hq & Ia & wdd5 & 5 & 02:30:18.037 & $-08: 22: 25.045$ & 0.669 & 0.006 & $\ldots$ & $\ldots$ & 0.04245 \\
\hline k442 & $\ldots$ & Unk & wcc3 & 10 & 02:06:19.076 & $-03: 58: 01.372$ & $\ldots$ & $\ldots$ & $\ldots$ & $\ldots$ & 0.02295 \\
\hline k443 & 2004hp & Unk & wcc1 & 4 & 02:09:35.522 & $-03: 46: 23.520$ & $\ldots$ & $\ldots$ & $\ldots$ & $\ldots$ & 0.02244 \\
\hline k444 & $\ldots$ & Gal & wdd5 & 2 & 02:30:00.763 & $-08: 37: 25.930$ & $\ldots$ & $\ldots$ & 0.1921 & 0.0002 & 0.03426 \\
\hline k448 & $2004 \mathrm{hr}$ & Ia & wbb6 & 2 & 01:08:48.336 & $+00: 00: 49.449$ & 0.405 & 0.007 & 0.4081 & 0.0001 & 0.03243 \\
\hline $\mathrm{k} 453$ & $\ldots$ & Gal & wdd8 & 13 & $02: 31: 36.957$ & $-08: 58: 10.962$ & $\ldots$ & $\ldots$ & 0.5418 & 0.0003 & 0.02825 \\
\hline k459 & $\ldots$ & Unk & wcc7 & 10 & 02:10:28.886 & $-05: 07: 11.337$ & $\ldots$ & $\ldots$ & $\ldots$ & $\ldots$ & 0.01991 \\
\hline k467 & $\ldots$ & Ia? & wdd 2 & 13 & $02: 31: 11.801$ & $-07: 47: 34.124$ & 0.607 & 0.008 & $\ldots$ & $\ldots$ & 0.02913 \\
\hline k472 & $\ldots$ & Gal & wcc3 & 15 & 02:06:29.663 & $-03: 33: 08.079$ & $\ldots$ & $\ldots$ & 0.1368 & 0.0002 & 0.02320 \\
\hline k485 & 2004hs & Ia & wcc4 & 6 & 02:09:33.689 & $-04: 13: 03.931$ & 0.417 & 0.003 & $\ldots$ & $\ldots$ & 0.02454 \\
\hline k490 & $\ldots$ & Ia & wdd 2 & 4 & $02: 30: 24.320$ & $-07: 53: 20.935$ & 0.709 & 0.001 & 0.7147 & 0.0009 & 0.03220 \\
\hline k505 & $\ldots$ & Gal & wcc3 & 13 & 02:06:11.608 & $-03: 44: 17.379$ & $\ldots$ & $\ldots$ & 0.2405 & 0.0001 & 0.02248 \\
\hline
\end{tabular}


Table 6

(Continued)

\begin{tabular}{|c|c|c|c|c|c|c|c|c|c|c|c|}
\hline ESSENCE ID & IAU ID & Type & Subfield & Amp & R.A. (J2000) & decl. & $z_{\text {SNID }}$ & $\sigma_{z}$ & $z_{\mathrm{GAL}}$ & $\sigma_{z_{\mathrm{GAL}}}$ & $\mathrm{MW}_{E(B-V)}$ \\
\hline k509 & $\ldots$ & Gal & waa5 & 10 & $23: 28: 31.296$ & $-09: 25: 39.880$ & $\ldots$ & $\ldots$ & 0.2064 & 0.0001 & 0.03928 \\
\hline m001 & $\ldots$ & $\mathrm{Ia}$ & wbb6 & 1 & 01:08:22.010 & $-00: 05: 46.654$ & 0.290 & 0.004 & $\ldots$ & $\ldots$ & 0.03584 \\
\hline $\mathrm{m} 002$ & $\ldots$ & Gal & waa6 & 15 & $23: 26: 06.272$ & $-09: 05: 22.919$ & $\ldots$ & $\ldots$ & 0.3545 & 0.0002 & 0.03602 \\
\hline $\mathrm{m} 003$ & $\ldots$ & IIP & wec9 & 15 & $02: 05: 27.303$ & $-04: 42: 53.777$ & $\ldots$ & $\ldots$ & 0.2014 & 0.0003 & 0.02508 \\
\hline m004 & $\ldots$ & Gal & wcc8 & 8 & 02:07:12.708 & $-04: 37: 27.898$ & $\ldots$ & $\ldots$ & 0.3834 & 0.0004 & 0.02205 \\
\hline m006 & $\ldots$ & $\mathrm{Ib} / \mathrm{c}$ ? & wdd 8 & 1 & $02: 30: 27.266$ & $-09: 16: 10.189$ & 0.051 & 0.001 & 0.0572 & 0.0002 & 0.02980 \\
\hline $\mathrm{m} 010$ & $\ldots$ & $\mathrm{Ib}$ & wdd 8 & 9 & $02: 31: 46.238$ & $-09: 16: 25.667$ & $\ldots$ & $\ldots$ & 0.2156 & 0.0003 & 0.02735 \\
\hline $\mathrm{m} 011$ & $\ldots$ & II & wcc5 & 16 & 02:08:06.229 & $-04: 03: 51.137$ & $\ldots$ & $\ldots$ & 0.2045 & 0.0003 & 0.02310 \\
\hline $\mathrm{m} 012$ & $\ldots$ & Gal & wdd3 & 16 & $02: 29: 13.485$ & $-07: 34: 07.335$ & $\ldots$ & $\ldots$ & 0.1138 & 0.0002 & 0.02954 \\
\hline $\mathrm{m} 014$ & $\ldots$ & II & wec5 & 3 & 02:07:12.911 & $-04: 26: 40.049$ & $\ldots$ & $\ldots$ & 0.1988 & 0.0001 & 0.02264 \\
\hline $\mathrm{m} 022$ & $\ldots$ & Ia & waa1 & 14 & 23:30:02.706 & $-08: 33: 36.539$ & 0.238 & 0.004 & $\ldots$ & $\ldots$ & 0.03070 \\
\hline $\mathrm{m} 025$ & $\ldots$ & Gal & waa6 & 11 & $23: 25: 11.601$ & $-09: 23: 41.144$ & $\ldots$ & $\ldots$ & 0.7008 & 0.0001 & 0.03662 \\
\hline $\mathrm{m} 026$ & $\ldots$ & $\mathrm{Ia}$ & waa5 & 12 & $23: 28: 39.960$ & $-09: 19: 49.986$ & 0.656 & 0.006 & 0.6548 & 0.0003 & 0.03742 \\
\hline $\mathrm{m} 027$ & $\ldots$ & Ia & wbb6 & 12 & 01:09:15.013 & $+00: 08: 14.797$ & 0.285 & 0.003 & 0.2885 & 0.0002 & 0.02834 \\
\hline $\mathrm{m} 028$ & $\ldots$ & Gal & wcc5 & 12 & 02:08:49.544 & $-04: 23: 12.189$ & $\ldots$ & $\ldots$ & 0.6075 & 0.0001 & 0.02094 \\
\hline $\mathrm{m} 032$ & $\ldots$ & Ia & waa7 & 2 & $23: 29: 35.343$ & $-09: 58: 46.304$ & 0.154 & 0.003 & $\ldots$ & $\ldots$ & 0.02911 \\
\hline $\mathrm{m} 034$ & $\ldots$ & Ia & wdd3 & 2 & $02: 27: 50.324$ & $-07: 59: 11.705$ & 0.562 & 0.006 & 0.5577 & 0.0002 & 0.03114 \\
\hline $\mathrm{m} 035$ & $\ldots$ & AGN & waal & 5 & $23: 28: 55.967$ & $-08: 38: 18.364$ & 1.497 & 0.009 & $\ldots$ & $\ldots$ & 0.03167 \\
\hline $\mathrm{m} 037$ & $\ldots$ & Gal & wdd3 & 10 & 02:29:03.971 & $-07: 59: 43.697$ & $\ldots$ & $\ldots$ & 0.2397 & 0.0002 & 0.02607 \\
\hline $\mathrm{m} 038$ & $\ldots$ & II & wcc9 & 6 & $02: 05: 10.823$ & $-04: 47: 13.982$ & $\ldots$ & $\ldots$ & 0.0507 & 0.0004 & 0.02412 \\
\hline m039 & $\ldots$ & Ia & wdd3 & 6 & $02: 28: 04.636$ & $-07: 42: 44.373$ & 0.249 & 0.003 & 0.2481 & 0.0002 & 0.03069 \\
\hline $\mathrm{m} 040$ & $\ldots$ & Ia & wdd3 & 6 & $02: 27: 30.201$ & $-07: 41: 49.985$ & 0.481 & 0.003 & $\ldots$ & $\ldots$ & 0.03237 \\
\hline m041 & $\ldots$ & IIP & wcc7 & 7 & 02:09:49.784 & $-04: 45: 10.513$ & 0.220 & 0.006 & $\ldots$ & $\ldots$ & 0.02231 \\
\hline $\mathrm{m} 042$ & $\ldots$ & Gal & waa6 & 3 & $23: 24: 23.781$ & $-09: 22: 12.082$ & $\ldots$ & $\ldots$ & 0.1254 & 0.0001 & 0.03098 \\
\hline $\mathrm{m} 043$ & $\ldots$ & Ia & waal & 1 & $23: 29: 51.729$ & $-08: 56: 46.084$ & 0.266 & 0.003 & 0.2654 & 0.0010 & 0.02926 \\
\hline $\mathrm{m} 057$ & $\ldots$ & Ia & wcc4 & 11 & $02: 10: 56.774$ & $-04: 27: 29.962$ & 0.183 & 0.004 & 0.1810 & 0.0002 & 0.01992 \\
\hline $\mathrm{m} 062$ & $\ldots$ & Ia & wbb3 & 10 & 01:09:52.911 & $+00: 36: 19.019$ & 0.316 & 0.004 & 0.3139 & 0.0001 & 0.02457 \\
\hline $\mathrm{m} 070$ & $\ldots$ & Ia & wdd4 & 7 & $02: 33: 46.821$ & $-08: 08: 26.888$ & 0.214 & 0.003 & 0.2122 & 0.0002 & 0.03753 \\
\hline $\mathrm{m} 075$ & $\ldots$ & $\mathrm{Ia}$ & waa3 & 7 & $23: 24: 42.288$ & $-08: 29: 08.021$ & 0.101 & 0.003 & 0.0996 & 0.0002 & 0.03620 \\
\hline $\mathrm{m} 078$ & $\ldots$ & Gal & wcc2 & 5 & 02:07:05.775 & $-03: 41: 28.385$ & $\ldots$ & $\ldots$ & 0.3951 & 0.0005 & 0.02350 \\
\hline m082 & $\ldots$ & Unk & wec2 2 & 4 & 02:07:13.757 & $-03: 49: 12.890$ & $\ldots$ & $\ldots$ & 0.3472 & 0.0002 & 0.02425 \\
\hline $\mathrm{m} 087$ & $\ldots$ & Ia & wdd4 & 3 & $02: 33: 37.004$ & $-08: 27: 32.462$ & 0.289 & 0.006 & 0.2870 & 0.0003 & 0.03448 \\
\hline m095 & $\ldots$ & AGN & wdd 2 & 3 & $02: 30: 26.427$ & $-07: 57: 27.227$ & 0.992 & 0.012 & $\ldots$ & $\ldots$ & 0.03305 \\
\hline $\mathrm{m} 111$ & $\ldots$ & $\mathrm{AGN}$ & wcc7 & 11 & $02: 10: 36.083$ & $-05: 00: 57.306$ & 1.001 & 0.004 & $\ldots$ & $\ldots$ & 0.02014 \\
\hline $\mathrm{m} 135$ & $\ldots$ & Gal & waal & 2 & $23: 29: 32.878$ & $-08: 51: 20.312$ & $\ldots$ & $\ldots$ & 0.2887 & 0.0001 & 0.02885 \\
\hline $\mathrm{m} 138$ & $\ldots$ & Ia & wbb3 & 3 & 01:08:56.340 & $+00: 39: 25.350$ & 0.585 & 0.004 & 0.5877 & 0.0001 & 0.02758 \\
\hline m139 & $\ldots$ & IIn & waa3 & 7 & $23: 23: 57.823$ & $-08: 27: 08.205$ & $\ldots$ & $\ldots$ & 0.2113 & 0.0001 & 0.03730 \\
\hline $\mathrm{m} 142$ & $\ldots$ & Unk & wbb1 & 12 & $01: 15: 17.691$ & $+00: 47: 17.513$ & $\ldots$ & $\ldots$ & $\ldots$ & $\ldots$ & 0.03129 \\
\hline $\mathrm{m} 158$ & $\ldots$ & Ia & waa6 & 3 & $23: 24: 03.540$ & $-09: 23: 18.267$ & 0.461 & 0.006 & $\ldots$ & $\ldots$ & 0.03056 \\
\hline $\mathrm{m} 161$ & $\ldots$ & Gal & wdd9 & 4 & $02: 28: 23.645$ & $-09: 03: 12.171$ & $\ldots$ & $\ldots$ & 0.2304 & 0.0001 & 0.02661 \\
\hline $\mathrm{m} 166$ & $\ldots$ & AGN & waal & 5 & $23: 29: 20.817$ & $-08: 36: 48.770$ & 0.304 & 0.002 & $\ldots$ & $\ldots$ & 0.02623 \\
\hline m193 & $\ldots$ & Ia & wdd3 & 14 & 02:28:52.199 & $-07: 42: 09.763$ & 0.336 & 0.006 & 0.3304 & 0.0002 & 0.02804 \\
\hline $\mathrm{m} 219$ & $\ldots$ & Gal & wdd4 & 5 & 02:34:28.090 & $-08: 15: 19.399$ & $\ldots$ & $\ldots$ & 0.3104 & 0.0003 & 0.03676 \\
\hline $\mathrm{m} 226$ & $\ldots$ & Ia & wcc9 & 16 & 02:06:03.688 & $-04: 39: 59.080$ & 0.674 & 0.008 & 0.6739 & 0.0013 & 0.02413 \\
\hline n244 & $\ldots$ & Unk & wdd3 & 7 & 02:28:11.797 & $-07: 36: 29.340$ & $\ldots$ & $\ldots$ & $\ldots$ & $\ldots$ & 0.02938 \\
\hline $\mathrm{n} 246$ & $\ldots$ & Ia? & wbb7 & 11 & $01: 14: 33.074$ & $-00: 26: 23.184$ & 0.503 & 0.005 & 0.7055 & 0.0005 & 0.03047 \\
\hline $\mathrm{n} 255$ & $\ldots$ & Gal & wcc7 & 5 & 02:09:05.303 & $-04: 53: 36.615$ & $\ldots$ & $\ldots$ & 0.1369 & 0.0002 & 0.02116 \\
\hline n256 & $\ldots$ & Ia & wdd3 & 5 & 02:28:09.012 & $-07: 47: 49.616$ & 0.620 & 0.007 & $\ldots$ & $\ldots$ & 0.02975 \\
\hline n258 & $\ldots$ & Ia & wec5 & 4 & 02:06:42.346 & $-04: 22: 36.982$ & 0.525 & 0.008 & 0.5191 & 0.0002 & 0.02257 \\
\hline n260 & $\ldots$ & $\mathrm{AGN}$ & wcc8 & 2 & 02:06:36.320 & $-05: 06: 45.964$ & 2.013 & 0.006 & $\ldots$ & $\ldots$ & 0.02087 \\
\hline $\mathrm{n} 261$ & $\ldots$ & AGN & wbb7 & 16 & $01: 14: 59.513$ & $-00: 05: 55.501$ & 3.540 & 0.040 & $\ldots$ & $\ldots$ & 0.03026 \\
\hline $\mathrm{n} 263$ & $\ldots$ & Ia & wec9 & 4 & 02:05:14.946 & $-04: 56: 39.087$ & 0.365 & 0.003 & $\ldots$ & $\ldots$ & 0.02569 \\
\hline n268 & $\ldots$ & Gal & wdd6 & 15 & 02:29:19.971 & $-08: 12: 02.467$ & $\ldots$ & $\ldots$ & 0.2791 & 0.0004 & 0.03090 \\
\hline $\mathrm{n} 271$ & $\ldots$ & IIP & wbb5 & 16 & 01:13:06.506 & $+00: 30: 04.835$ & 0.236 & 0.003 & $\ldots$ & $\ldots$ & 0.03371 \\
\hline n278 & $\ldots$ & Ia & waa5 & 11 & $23: 28: 17.550$ & $-09: 23: 12.360$ & 0.308 & 0.006 & 0.3037 & 0.0002 & 0.03983 \\
\hline n284 & $\ldots$ & AGN & waa1 & 8 & $23: 29: 38.374$ & $-08: 21: 32.166$ & 1.990 & 0.008 & $\ldots$ & $\ldots$ & 0.02812 \\
\hline $\mathrm{n} 285$ & $\ldots$ & Ia & waa3 & 8 & $23: 23: 51.357$ & $-08: 23: 18.503$ & 0.531 & 0.009 & 0.5325 & 0.0001 & 0.03738 \\
\hline n295 & $\ldots$ & AGN & waa3 & 3 & $23: 24: 03.280$ & $-08: 44: 36.907$ & 1.235 & 0.007 & $\ldots$ & $\ldots$ & 0.03854 \\
\hline n312 & $\ldots$ & Gal & wdd9 & 14 & $02: 28: 45.104$ & $-08: 55: 47.232$ & $\ldots$ & $\ldots$ & 0.2860 & 0.0002 & 0.02937 \\
\hline n322 & $\ldots$ & Ia & wdd 9 & 12 & 02:29:00.487 & $-09: 02: 52.992$ & 0.753 & 0.006 & $\ldots$ & $\ldots$ & 0.02713 \\
\hline n326 & $\ldots$ & $\mathrm{Ia}$ & waal & 10 & $23: 29: 58.590$ & $-08: 53: 12.468$ & 0.267 & 0.006 & 0.2637 & 0.0002 & 0.02986 \\
\hline n346 & $\ldots$ & IIn & waal & 3 & $23: 28: 58.301$ & $-08: 46: 52.839$ & $\ldots$ & $\ldots$ & 0.2661 & 0.0002 & 0.02994 \\
\hline n368 & $\ldots$ & Ia & waa7 & 9 & $23: 30: 32.013$ & $-10: 03: 22.140$ & 0.342 & 0.006 & 0.3419 & 0.0002 & 0.02315 \\
\hline n395 & $\ldots$ & Gal & wcc8 & 7 & 02:07:32.469 & $-04: 42: 10.706$ & $\ldots$ & $\ldots$ & 0.4617 & 0.0002 & 0.02208 \\
\hline
\end{tabular}


Table 6

(Continued)

\begin{tabular}{|c|c|c|c|c|c|c|c|c|c|c|c|}
\hline ESSENCE ID & IAU ID & Type & Subfield & Amp & R.A. (J2000) & decl. & $z_{\mathrm{SNID}}$ & $\sigma_{z}$ & $z_{\mathrm{GAL}}$ & $\sigma_{z_{\mathrm{GAL}}}$ & $\mathrm{MW}_{E(B-V)}$ \\
\hline $\mathrm{n} 400$ & $\ldots$ & Ia & wbb8 & 12 & $01: 13: 13.258$ & $-00: 23: 25.853$ & 0.421 & 0.007 & 0.4250 & 0.0001 & 0.03102 \\
\hline n404 & $\ldots$ & Ia & wdd8 & 14 & $02: 31: 31.433$ & $-08: 55: 11.512$ & 0.211 & 0.005 & $\ldots$ & $\ldots$ & 0.02875 \\
\hline $\mathrm{n} 406$ & $\ldots$ & Ia? & wdd8 & 16 & $02: 31: 19.601$ & $-08: 45: 09.787$ & 0.7700 & 0.01 & $\ldots$ & $\ldots$ & 0.02933 \\
\hline n408 & $\ldots$ & Gal & wbb9 & 16 & 01:09:49.036 & $-00: 07: 42.929$ & $\ldots$ & $\ldots$ & 0.9198 & 0.0003 & 0.03468 \\
\hline p415 & $\ldots$ & Gal & waa3 & 16 & $23: 26: 02.504$ & $-08: 21: 10.846$ & $\ldots$ & $\ldots$ & 0.3434 & 0.0002 & 0.02900 \\
\hline $\mathrm{p} 425$ & $\ldots$ & Ia & waal & 14 & $23: 29: 56.189$ & $-08: 34: 24.400$ & 0.456 & 0.004 & 0.4583 & 0.0001 & 0.03095 \\
\hline p429 & $\ldots$ & Ia? & waa3 & 13 & $23: 26: 02.216$ & $-08: 35: 47.978$ & $\ldots$ & $\ldots$ & 0.5482 & 0.0002 & 0.02983 \\
\hline p434 & $\ldots$ & Ia & wbb5 & 12 & $01: 12: 40.253$ & $+00: 14: 56.591$ & $\ldots$ & $\ldots$ & 0.3383 & 0.0004 & 0.03441 \\
\hline p444 & $\ldots$ & Ia & wcc2 2 & 5 & 02:06:36.165 & $-03: 41: 33.614$ & 0.633 & 0.004 & $\ldots$ & $\ldots$ & 0.02423 \\
\hline p445 & $\ldots$ & Ia & wbb1 & 4 & $01: 13: 14.547$ & $+00: 48: 47.659$ & 0.816 & 0.002 & 0.8069 & 0.0002 & 0.02458 \\
\hline p454 & $\ldots$ & Ia & wec2 2 & 15 & 02:08:32.461 & $-03: 33: 34.241$ & 0.691 & 0.008 & $\ldots$ & $\ldots$ & 0.02282 \\
\hline $\mathrm{p} 455$ & $\ldots$ & Ia & wcc4 & 15 & 02:11:00.014 & $-04: 09: 37.601$ & 0.285 & 0.006 & 0.2974 & 0.0002 & 0.02023 \\
\hline p458 & $\ldots$ & Unk & waa3 & 10 & $23: 25: 30.268$ & $-08: 52: 04.940$ & $\ldots$ & $\ldots$ & $\ldots$ & $\ldots$ & 0.03947 \\
\hline p459 & $\ldots$ & Ia & wcc4 & 10 & 02:10:20.082 & $-04: 33: 13.440$ & 0.702 & 0.004 & $\ldots$ & $\ldots$ & 0.02173 \\
\hline p461 & $\ldots$ & Gal & waa5 & 6 & $23: 26: 42.317$ & $-09: 07: 28.646$ & $\ldots$ & $\ldots$ & 0.4075 & 0.0005 & 0.03451 \\
\hline p520 & $\ldots$ & Ia? & wcc2 2 & 12 & 02:08:09.339 & $-03: 48: 04.967$ & $\ldots$ & $\ldots$ & $\ldots$ & $\ldots$ & 0.02298 \\
\hline p521 & $\ldots$ & Gal & wcc7 & 14 & $02: 10: 17.525$ & $-04: 46: 52.214$ & $\ldots$ & $\ldots$ & 0.3053 & 0.0002 & 0.02171 \\
\hline p524 & $\ldots$ & Ia & wdd8 & 6 & $02: 30: 10.156$ & $-08: 52: 50.856$ & 0.516 & 0.004 & $\ldots$ & $\ldots$ & 0.03528 \\
\hline p527 & $\ldots$ & Ia? & wcc2 & 15 & 02:08:10.469 & $-03: 32: 17.637$ & $\ldots$ & $\ldots$ & 0.4351 & 0.0002 & 0.02451 \\
\hline p528 & $\ldots$ & Ia & wcc2 2 & 8 & 02:07:04.661 & $-03: 28: 04.268$ & 0.780 & 0.009 & 0.7812 & 0.0001 & 0.02355 \\
\hline p534 & $\ldots$ & Ia & wcc3 & 4 & 02:04:56.094 & $-03: 49: 03.645$ & 0.610 & 0.005 & 0.6202 & 0.0011 & 0.02438 \\
\hline p535 & $\ldots$ & Unk & wec5 & 13 & 02:08:28.123 & $-04: 16: 34.893$ & $\ldots$ & $\ldots$ & $\ldots$ & $\ldots$ & 0.02216 \\
\hline $\mathrm{q} 002$ & $\ldots$ & Ia & wcc3 & 6 & $02: 05: 12.945$ & $-03: 39: 00.723$ & 0.350 & 0.003 & 0.3469 & 0.0002 & 0.02326 \\
\hline q006 & $\ldots$ & Ia? & wcc1 & 10 & $02: 10: 52.276$ & $-03: 57: 39.374$ & 0.290 & 0.010 & $\ldots$ & $\ldots$ & 0.02208 \\
\hline q007 & $20061 w$ & Ia & wec2 2 & 10 & 02:08:33.670 & $-03: 57: 12.888$ & 0.210 & 0.004 & 0.2135 & 0.0002 & 0.02401 \\
\hline q008 & 2006ly & Ia? & wdd4 & 10 & $02: 34: 42.381$ & $-08: 30: 39.885$ & 0.287 & 0.010 & 0.2913 & 0.0002 & 0.03191 \\
\hline $\mathrm{q} 014$ & $2006 \mathrm{~lx}$ & Ia & wdd5 & 8 & 02:30:10.389 & $-08: 06: 54.094$ & 0.270 & 0.003 & 0.2693 & 0.0002 & 0.03147 \\
\hline q018 & $\ldots$ & Ia? & wdd5 & 14 & $02: 31: 39.017$ & $-08: 18: 05.170$ & 0.270 & 0.010 & $\ldots$ & $\ldots$ & 0.03499 \\
\hline q021 & $\ldots$ & Ia? & wdd4 & 7 & 02:33:43.919 & $-08: 05: 50.050$ & 0.360 & 0.010 & $\ldots$ & $\ldots$ & 0.03818 \\
\hline $\mathrm{q} 022$ & $\ldots$ & Ia? & wbb5 & 9 & $01: 12: 03.875$ & $-00: 01: 29.045$ & 0.226 & 0.010 & $\ldots$ & $\ldots$ & 0.03038 \\
\hline q031 & $\ldots$ & Gal & wbb6 & 2 & 01:08:42.081 & $-00: 00: 57.201$ & $\ldots$ & $\ldots$ & 0.2697 & 0.0002 & 0.03289 \\
\hline q036 & $2006 \mathrm{lz}$ & II-pec & wdd6 & 8 & $02: 27: 40.742$ & $-08: 10: 08.182$ & 0.179 & 0.006 & $\ldots$ & $\ldots$ & 0.02762 \\
\hline q048 & $2006 \mathrm{ma}$ & Ia & wbb7 & 11 & $01: 15: 11.657$ & $-00: 28: 03.151$ & 0.440 & 0.010 & 0.4371 & 0.0007 & 0.03018 \\
\hline q049 & $2006 \mathrm{mc}$ & Ia & wdd8 & 11 & $02: 32: 02.611$ & $-09: 07: 21.181$ & 0.421 & 0.005 & 0.4204 & 0.0001 & 0.02963 \\
\hline q054 & $2006 \mathrm{mb}$ & Ia & wdd8 & 5 & 02:30:54.298 & $-08: 57: 42.078$ & 0.331 & 0.005 & 0.3275 & 0.0003 & 0.02705 \\
\hline q060 & $\ldots$ & IIP & wbb1 & 11 & $01: 14: 48.945$ & $+00: 44: 47.120$ & $\ldots$ & $\ldots$ & 0.1441 & 0.0002 & 0.03191 \\
\hline q061 & $2006 \mathrm{me}$ & Ia & wbb4 & 11 & 01:14:47.189 & $+00: 10: 13.284$ & 0.302 & 0.005 & 0.2996 & 0.0001 & 0.03176 \\
\hline q067 & $2006 \mathrm{mf}$ & Ia & wdd2 & 3 & $02: 30: 37.318$ & $-07: 57: 04.538$ & 0.187 & 0.007 & 0.1824 & 0.0003 & 0.03380 \\
\hline q069 & $2006 \mathrm{md}$ & Ia? & wbb5 & 6 & $01: 11: 31.492$ & $+00: 24: 34.163$ & 0.262 & 0.010 & 0.2470 & 0.0004 & 0.02595 \\
\hline q070 & $\ldots$ & Gal & wdd6 & 6 & 02:28:04.925 & $-08: 15: 40.481$ & $\ldots$ & $\ldots$ & 0.1256 & 0.0003 & 0.02960 \\
\hline q075 & $2006 \mathrm{mg}$ & Ia & wdd 2 & 9 & $02: 31: 37.750$ & $-08: 06: 40.098$ & 0.427 & 0.005 & $\ldots$ & $\ldots$ & 0.04023 \\
\hline q102 & $2006 \mathrm{mh}$ & Ia? & wbb4 & 14 & $01: 15: 13.398$ & $+00: 23: 57.312$ & 0.435 & 0.010 & 0.4359 & 0.0008 & 0.02898 \\
\hline q106 & $2006 \mathrm{mk}$ & Ia & wdd5 & 1 & $02: 30: 16.911$ & $-08: 40: 47.345$ & 0.477 & 0.004 & 0.4754 & 0.0001 & 0.03976 \\
\hline q107 & $2006 \mathrm{mj}$ & Ia & wcc1 & 8 & 02:09:03.042 & $-03: 28: 27.832$ & 0.650 & 0.009 & 0.6514 & 0.0001 & 0.02406 \\
\hline q108 & $2006 \mathrm{mi}$ & Ia & wcc3 & 9 & 02:05:55.040 & $-04: 00: 53.216$ & 0.622 & 0.005 & 0.6231 & 0.0001 & 0.02476 \\
\hline q112 & $2006 \mathrm{ml}$ & Ia & wbb9 & 3 & 01:08:43.977 & $-00: 31: 36.593$ & 0.637 & 0.003 & $\ldots$ & $\ldots$ & 0.03642 \\
\hline q114 & $2006 \mathrm{~mm}$ & Ia & wbb6 & 6 & 01:08:48.662 & $+00: 17: 22.315$ & 0.701 & 0.011 & 0.6875 & 0.0002 & 0.02730 \\
\hline q125 & $2006 \mathrm{mn}$ & Ia & wbb6 & 1 & 01:07:48.392 & $-00: 06: 35.454$ & 0.347 & 0.004 & 0.3486 & 0.0004 & 0.03112 \\
\hline q150 & $\ldots$ & Unk & wdd5 & 16 & $02: 31: 18.622$ & $-08: 07: 11.563$ & $\ldots$ & $\ldots$ & $\ldots$ & $\ldots$ & 0.03639 \\
\hline r184 & 2006 sa & IIP & wec9 & 6 & 02:05:14.944 & $-04: 48: 51.685$ & $\ldots$ & $\ldots$ & 0.1609 & 0.0002 & 0.02442 \\
\hline r185 & $\ldots$ & Ia & wbb8 & 3 & $01: 11: 48.238$ & $-00: 29: 49.579$ & 0.179 & 0.006 & 0.1800 & 0.0003 & 0.02423 \\
\hline r186 & $2006 s b$ & Ia & wec5 & 8 & 02:06:30.312 & $-04: 05: 30.553$ & 0.313 & 0.004 & 0.3126 & 0.0001 & 0.02101 \\
\hline r190 & $2006 s c$ & Ia & wcc7 & 15 & $02: 10: 10.226$ & $-04: 44: 12.545$ & 0.355 & 0.007 & 0.3568 & 0.0002 & 0.02261 \\
\hline r192 & $\ldots$ & Gal & wec9 & 13 & 02:05:23.959 & $-04: 52: 16.485$ & $\ldots$ & $\ldots$ & 0.6336 & 0.0001 & 0.02426 \\
\hline r193 & $2006 \mathrm{sm}$ & Ia & wdd4 & 2 & $02: 33: 29.487$ & $-08: 30: 11.879$ & 0.609 & 0.005 & $\ldots$ & $\ldots$ & 0.03296 \\
\hline r195 & 2006 si & $\mathrm{Ia}$ & wcc1 & 5 & 02:09:51.320 & $-03: 43: 32.520$ & 0.542 & 0.008 & 0.5424 & 0.0001 & 0.02335 \\
\hline r196 & 2006 sh & IIn & wcc1 & 5 & 02:09:11.064 & $-03: 44: 42.104$ & 0.260 & 0.010 & 0.2639 & 0.0001 & 0.02275 \\
\hline r199 & 2006sl & Ia? & wdd7 & 6 & $02: 32: 15.952$ & $-08: 48: 34.335$ & 0.410 & 0.010 & 0.4180 & 0.0002 & 0.02808 \\
\hline r200 & 2006sd & Ia & wbb1 & 7 & $01: 14: 24.151$ & $+01: 02: 39.488$ & 0.283 & 0.007 & $\ldots$ & $\ldots$ & 0.03124 \\
\hline r204 & $\ldots$ & Gal & wcc1 & 7 & 02:09:47.393 & $-03: 34: 25.676$ & $\ldots$ & $\ldots$ & 0.4212 & 0.0001 & 0.02441 \\
\hline r205 & $\ldots$ & IIn & wcc1 & 7 & 02:09:37.948 & $-03: 31: 20.238$ & $\ldots$ & $\ldots$ & 0.0517 & 0.0004 & 0.02574 \\
\hline r206 & 2006 se & Ia & wbb4 & 11 & $01: 14: 48.050$ & $+00: 06: 39.370$ & 0.610 & 0.010 & 0.6108 & 0.0003 & 0.03126 \\
\hline r207 & $2006 s f$ & Ia & wcc2 2 & 11 & 02:08:11.658 & $-03: 51: 40.230$ & 0.560 & 0.010 & 0.5616 & 0.0006 & 0.02335 \\
\hline
\end{tabular}


Table 6

(Continued)

\begin{tabular}{|c|c|c|c|c|c|c|c|c|c|c|c|}
\hline ESSENCE ID & IAU ID & Type & Subfield & Amp & R.A. (J2000) & decl. & $z_{\text {SNID }}$ & $\sigma_{z}$ & $z_{\mathrm{GAL}}$ & $\sigma_{z_{\mathrm{GAL}}}$ & $\mathrm{MW}_{E(B-V)}$ \\
\hline r209 & 2006 sg & Ia & wcc2 2 & 12 & 02:08:13.041 & $-03: 46: 21.937$ & 0.428 & 0.003 & 0.4451 & 0.0002 & 0.02290 \\
\hline r212 & $2006 \mathrm{sj}$ & Ia & wcc1 & 15 & 02:10:22.419 & $-03: 33: 09.269$ & $\ldots$ & $\ldots$ & 0.6535 & 0.0004 & 0.02468 \\
\hline $\mathrm{r} 213$ & 2006 sk & Ia & wcc4 & 16 & 02:10:33.800 & $-04: 04: 03.845$ & 0.321 & 0.008 & 0.3270 & 0.0001 & 0.02228 \\
\hline $\mathrm{r} 215$ & $\ldots$ & Unk & wcc7 & 4 & 02:08:55.716 & $-04: 59: 46.063$ & $\ldots$ & $\ldots$ & $\ldots$ & $\ldots$ & 0.02095 \\
\hline $\mathrm{r} 225$ & 2006 sn & Ia & wec9 & 13 & 02:06:18.251 & $-04: 51: 33.148$ & 0.415 & 0.006 & 0.4149 & 0.0001 & 0.02228 \\
\hline r230 & 2006so & Ia & wdd7 & 12 & $02: 33: 49.152$ & $-08: 59: 15.768$ & 0.259 & 0.005 & $\ldots$ & $\ldots$ & 0.03030 \\
\hline r311 & 2006 sp & Ia & wbb8 & 3 & $01: 10: 55.171$ & $-00: 27: 52.289$ & $\ldots$ & $\ldots$ & 0.7989 & 0.0002 & 0.02510 \\
\hline r314 & $\ldots$ & Gal & wcc4 & 5 & 02:09:59.003 & $-04: 18: 53.926$ & $\ldots$ & $\ldots$ & 0.1092 & 0.0003 & 0.02445 \\
\hline r317 & $\ldots$ & Ia & wbb1 & 5 & $01: 13: 24.658$ & $+00: 51: 27.757$ & 0.736 & 0.005 & 0.3361 & 0.0004 & 0.02550 \\
\hline r318 & 2006 sq & Ia & wcc2 2 & 2 & $02: 07: 11.267$ & $-03: 57: 07.942$ & 0.222 & 0.002 & $\ldots$ & $\ldots$ & 0.02526 \\
\hline r322 & $2006 \mathrm{tg}$ & Ia & wcc9 & 8 & 02:04:14.168 & $-04: 40: 18.623$ & 0.521 & 0.010 & 0.5138 & 0.0002 & 0.02531 \\
\hline r328 & 2006th & II & waal & 1 & $23: 29: 00.954$ & $-08: 54: 04.953$ & $\ldots$ & $\ldots$ & 0.1463 & 0.0002 & 0.03533 \\
\hline r329 & $\ldots$ & Gal & wcc2 2 & 16 & 02:07:48.017 & $-03: 29: 12.632$ & $\ldots$ & $\ldots$ & 0.6209 & 0.0001 & 0.02405 \\
\hline r331 & $\ldots$ & Gal & waal & 14 & $23: 30: 49.841$ & $-08: 32: 37.728$ & $\ldots$ & $\ldots$ & 0.4225 & 0.0003 & 0.02973 \\
\hline r334 & $2006 \mathrm{ti}$ & II & waal & 3 & $23: 29: 13.133$ & $-08: 47: 57.762$ & $\ldots$ & $\ldots$ & 0.2051 & 0.0004 & 0.02897 \\
\hline s340 & $2006 \mathrm{tj}$ & Ia & wbb3 & 4 & 01:09:23.284 & $+00: 42: 42.313$ & 0.528 & 0.006 & $\ldots$ & $\ldots$ & 0.02954 \\
\hline s346 & 2006tl & Ia & wbb9 & 1 & 01:09:17.282 & $-00: 40: 27.967$ & 0.270 & 0.010 & 0.2721 & 0.0006 & 0.03367 \\
\hline s347 & $2006 \mathrm{tk}$ & Ia & wbb6 & 8 & 01:07:52.640 & $+00: 27: 55.293$ & 0.313 & 0.003 & $\ldots$ & $\ldots$ & 0.02954 \\
\hline s349 & $2006 \mathrm{tm}$ & Ia & wbb6 & 12 & 01:09:17.297 & $+00: 09: 11.389$ & 0.220 & 0.007 & 0.2156 & 0.0002 & 0.02782 \\
\hline s350 & 2006to & Ia & wcc2 2 & 1 & 02:07:34.387 & $-04: 00: 04.177$ & 0.682 & 0.010 & 0.6834 & 0.0001 & 0.02453 \\
\hline s351 & 2006tp & Ia & wcc4 & 1 & 02:09:14.040 & $-04: 37: 11.970$ & 0.720 & 0.006 & 0.7275 & 0.0002 & 0.02118 \\
\hline s353 & 2006 tr & Ia? & wdd3 & 4 & $02: 28: 29.532$ & $-07: 53: 28.493$ & 0.581 & 0.010 & 0.5956 & 0.0002 & 0.02658 \\
\hline s354 & $\ldots$ & Ia? & wec 9 & 10 & 02:06:10.482 & $-05: 05: 23.000$ & $\ldots$ & $\ldots$ & 0.5588 & 0.0004 & 0.02130 \\
\hline s355 & $2006 \mathrm{tn}$ & Ia & wcc 9 & 10 & 02:05:36.019 & $-05: 08: 46.272$ & 0.670 & 0.010 & 0.6734 & 0.0002 & 0.02461 \\
\hline s362 & $2006 \mathrm{tq}$ & Ib-pec & wcc 4 & 8 & 02:10:00.697 & $-04: 06: 00.903$ & 0.262 & 0.001 & 0.2622 & 0.0001 & 0.02390 \\
\hline s370 & 2006tu & Ia & wdd 2 & 2 & $02: 29: 56.534$ & $-07: 59: 50.850$ & 0.439 & 0.005 & 0.4394 & 0.0002 & 0.03014 \\
\hline s371 & $\ldots$ & II? & wbb8 & 11 & $01: 12: 57.431$ & $-00: 26: 54.525$ & $\ldots$ & $\ldots$ & 0.2499 & 0.0001 & 0.02858 \\
\hline s372 & $\ldots$ & Ia & wcc 2 & 16 & $02: 08: 24.257$ & $-03: 27: 32.636$ & 0.706 & 0.010 & 0.7076 & 0.0014 & 0.02480 \\
\hline s373 & $2006 \mathrm{tt}$ & $\mathrm{Ia}$ & wcc4 & 12 & 02:10:47.935 & $-04: 24: 56.952$ & 0.630 & 0.003 & $\ldots$ & $\ldots$ & 0.02041 \\
\hline s374 & $2006 \mathrm{tv}$ & Ia? & wdd4 & 9 & $02: 35: 34.225$ & $-08: 34: 22.069$ & 0.757 & 0.010 & 0.7581 & 0.0002 & 0.02855 \\
\hline s375 & 2006ts & $\mathrm{IaT}$ & wccl & 15 & $02: 10: 18.687$ & $-03: 32: 26.335$ & 0.551 & 0.006 & 0.5569 & 0.0003 & 0.02473 \\
\hline s377 & $2006 \mathrm{tw}$ & $\mathrm{IaT}$ & wbb6 & 6 & $01: 08: 54.227$ & $+00: 17: 56.510$ & $\ldots$ & $\ldots$ & 0.3987 & 0.0005 & 0.02658 \\
\hline s378 & $2006 t x$ & SN? & wbb9 & 11 & 01:10:26.793 & $-00: 34: 07.640$ & $\ldots$ & $\ldots$ & 0.5005 & 0.0002 & 0.02851 \\
\hline s379 & 2006ty & Ia & wbb3 & 10 & $01: 10: 45.361$ & $+00: 34: 04.408$ & 0.181 & 0.004 & 0.1923 & 0.0001 & 0.02689 \\
\hline s380 & $\ldots$ & Ia & wec7 & 2 & 02:09:00.048 & $-05: 07: 41.787$ & $\ldots$ & $\ldots$ & 0.6355 & 0.0002 & 0.02096 \\
\hline s383 & $2006 \mathrm{tz}$ & SN? & wcc 4 & 13 & $02: 10: 27.034$ & $-04: 17: 08.159$ & $\ldots$ & $\ldots$ & 0.3920 & 0.0001 & 0.02371 \\
\hline $\mathrm{x} 005$ & $2007 \mathrm{sz}$ & IIP & wcc8 & 2 & 02:07:00.230 & $-05: 06: 08.212$ & 0.046 & 0.005 & $\ldots$ & $\ldots$ & 0.02235 \\
\hline x016 & $2007 \mathrm{td}$ & IIn & wcc3 & 5 & 02:04:26.895 & $-03: 44: 18.859$ & $\ldots$ & $\ldots$ & 0.3442 & 0.0002 & 0.02666 \\
\hline x017 & $2007 \mathrm{ta}$ & $\mathrm{Ia}$ & wbb4 & 1 & $01: 13: 15.801$ & $-00: 01: 31.428$ & 0.418 & 0.006 & 0.4222 & 0.0004 & 0.02770 \\
\hline $\mathrm{x} 020$ & 2007 te & Ia & wcc1 & 7 & 02:09:29.402 & $-03: 35: 35.054$ & 0.686 & 0.008 & $\ldots$ & $\ldots$ & 0.02540 \\
\hline $\mathrm{x} 022$ & 2007 sx & IIP & wbb9 & 9 & 01:10:09.249 & $-00: 42: 08.035$ & 0.12 & 0.01 & 0.1171 & 0.0004 & 0.03418 \\
\hline x024 & 2007sy & II? & wbb1 & 12 & $01: 15: 25.989$ & $+00: 49: 06.597$ & 0.19 & 0.01 & 0.1936 & 0.0002 & 0.03165 \\
\hline $\mathrm{x} 025$ & $2007 \mathrm{tb}$ & Ia & wbb1 & 12 & $01: 14: 41.252$ & $+00: 46: 51.824$ & 0.372 & 0.008 & 0.3553 & 0.0001 & 0.03295 \\
\hline $\mathrm{x} 027$ & $\ldots$ & Unk & wcc2 & 12 & 02:08:15.486 & $-03: 49: 35.804$ & $\ldots$ & $\ldots$ & $\ldots$ & $\ldots$ & 0.02259 \\
\hline $\mathrm{x} 028$ & $2007 \mathrm{tc}$ & Ia & wbb4 & 13 & $01: 14: 46.894$ & $+00: 17: 06.778$ & 0.609 & 0.007 & 0.600 & 0.0010 & 0.03209 \\
\hline $\mathrm{x} 033$ & $2007 \mathrm{tf}$ & Ia & wbb6 & 10 & 01:09:59.349 & $-00: 01: 06.947$ & 0.405 & 0.007 & 0.4062 & 0.0012 & 0.03114 \\
\hline $\mathrm{x} 034$ & 2007th & Ia & wcc7 & 11 & 02:10:57.582 & $-05: 00: 24.789$ & 0.508 & 0.003 & $\ldots$ & $\ldots$ & 0.02021 \\
\hline $\mathrm{x} 035$ & $\ldots$ & Gal & wcc 9 & 11 & 02:05:24.988 & $-05: 03: 33.228$ & $\ldots$ & $\ldots$ & 0.4029 & 0.0002 & 0.02415 \\
\hline x038 & $2007 \operatorname{tg}$ & $\mathrm{Ia}$ & wec 9 & 15 & 02:06:10.505 & $-04: 42: 23.286$ & 0.512 & 0.008 & $\ldots$ & $\ldots$ & 0.02367 \\
\hline $\mathrm{x} 039$ & $\ldots$ & Ia & wdd3 & 15 & $02: 29: 18.063$ & $-07: 39: 01.433$ & 0.771 & 0.004 & $\ldots$ & $\ldots$ & 0.02975 \\
\hline $\mathrm{x} 055$ & $2007 \mathrm{tk}$ & Ia? & wdd5 & 6 & 02:30:24.609 & $-08: 17: 54.401$ & $\ldots$ & $\ldots$ & 0.3495 & 0.0002 & 0.03726 \\
\hline x066 & $2007 \mathrm{tj}$ & Ia & wcc4 & 14 & 02:11:04.611 & $-04: 11: 50.056$ & 0.331 & 0.003 & 0.3286 & 0.0002 & 0.02047 \\
\hline x068 & $\ldots$ & Gal & wdd5 & 14 & $02: 31: 12.905$ & $-08: 18: 18.552$ & $\ldots$ & $\ldots$ & 0.4350 & 0.0002 & 0.03451 \\
\hline $\mathrm{x} 071$ & $2007 \mathrm{ti}$ & Ia & wec7 & 16 & 02:10:09.836 & $-04: 39: 49.342$ & 0.476 & 0.008 & 0.4865 & 0.0002 & 0.02324 \\
\hline $\mathrm{x} 072$ & $\ldots$ & Gal & wcc 8 & 16 & 02:08:37.294 & $-04: 37: 35.354$ & $\ldots$ & $\ldots$ & 0.1919 & 0.0001 & 0.02107 \\
\hline $\mathrm{x} 076$ & $2007 \mathrm{tn}$ & II & wdd6 & 2 & $02: 28: 14.790$ & $-08: 36: 32.198$ & $\ldots$ & $\ldots$ & $\ldots$ & $\ldots$ & 0.03026 \\
\hline x077 & $2007 t p$ & Ia & wdd 8 & 2 & $02: 30: 23.940$ & $-09: 13: 39.846$ & 0.517 & 0.009 & 0.520 & 0.0010 & 0.03026 \\
\hline x080 & $2007 \mathrm{tl}$ & Ia & wbb8 & 6 & 01:11:04.912 & $-00: 15: 43.364$ & 0.374 & 0.008 & 0.3731 & 0.0002 & 0.02838 \\
\hline $\mathrm{x} 084$ & $\ldots$ & Gal? & wdd3 & 12 & $02: 29: 20.643$ & $-07: 51: 00.932$ & $\ldots$ & $\ldots$ & $\ldots$ & $\ldots$ & 0.02666 \\
\hline $\mathrm{x} 085$ & 2007 to & Ia & wdd9 & 12 & $02: 29: 42.061$ & $-09: 02: 05.252$ & 0.648 & 0.008 & 0.6345 & 0.0001 & 0.03208 \\
\hline x089 & $2007 \mathrm{tm}$ & $\mathrm{IaT}$ & wcc3 & 15 & 02:06:04.748 & $-03: 32: 29.261$ & 0.5 & 0.05 & 0.4919 & 0.0001 & 0.02307 \\
\hline x093 & $\ldots$ & Ia & wdd 9 & 11 & $02: 29: 43.134$ & $-09: 06: 54.931$ & $\ldots$ & $\ldots$ & 0.5004 & 0.0001 & 0.03314 \\
\hline $\mathrm{x} 103$ & $\ldots$ & Ia? & wbb5 & 4 & 01:10:48.009 & $+00: 13: 36.806$ & $\ldots$ & $\ldots$ & $\ldots$ & $\ldots$ & 0.02585 \\
\hline x107 & $2007 \mathrm{tq}$ & Ia & wdd3 & 12 & $02: 29: 23.345$ & $-07: 52: 27.686$ & 0.145 & 0.002 & 0.1462 & 0.0009 & 0.02682 \\
\hline
\end{tabular}


Table 6

(Continued)

\begin{tabular}{|c|c|c|c|c|c|c|c|c|c|c|c|}
\hline ESSENCE ID & IAU ID & Type & Subfield & Amp & R.A. (J2000) & decl. & $z_{\text {SNID }}$ & $\sigma_{z}$ & $z_{\mathrm{GAL}}$ & $\sigma_{z_{\mathrm{GAL}}}$ & $\mathrm{MW}_{E(B-V)}$ \\
\hline x113 & $\ldots$ & II? & wdd4 & 16 & $02: 34: 36.128$ & $-08: 01: 11.116$ & $\ldots$ & $\ldots$ & $\ldots$ & $\ldots$ & 0.03962 \\
\hline y117 & $\ldots$ & Unk & wcc5 & 16 & 02:08:14.332 & $-04: 05: 09.772$ & $\ldots$ & $\ldots$ & $\ldots$ & $\ldots$ & 0.02299 \\
\hline y118 & $2007 \mathrm{tu}$ & IIP & wbb7 & 1 & 01:14:05.219 & $-00: 36: 25.629$ & 0.222 & 0.007 & $\ldots$ & $\ldots$ & 0.02995 \\
\hline y122 & $2007 \mathrm{tx}$ & Ic & wdd6 & 2 & $02: 28: 33.314$ & $-08: 35: 25.690$ & 0.674 & 0.006 & 0.6764 & 0.0001 & 0.03035 \\
\hline y123 & $\ldots$ & Gal & wdd7 & 2 & $02: 32: 56.953$ & $-09: 08: 21.287$ & $\ldots$ & $\ldots$ & 0.1939 & 0.0007 & 0.02755 \\
\hline y125 & $2007 \mathrm{tv}$ & Ia & wcc9 & 3 & 02:05:13.326 & $-05: 01: 42.415$ & 0.310 & 0.006 & 0.3108 & 0.0003 & 0.02459 \\
\hline y126 & $\ldots$ & Gal & wec9 & 3 & 02:04:27.408 & $-05: 01: 59.892$ & $\ldots$ & $\ldots$ & 0.7944 & 0.0002 & 0.02355 \\
\hline y127 & 2007 ty & Ia & wdd6 & 5 & $02: 28: 34.381$ & $-08: 23: 49.491$ & 0.518 & 0.003 & $\ldots$ & $\ldots$ & 0.03131 \\
\hline y131 & 2007 tw & $\mathrm{SN} ?$ & wec9 & 11 & 02:05:32.964 & $-05: 02: 46.626$ & $\ldots$ & $\ldots$ & 0.6654 & 0.0001 & 0.02404 \\
\hline y134 & 2007 ts & Ia & wbb6 & 8 & 01:07:58.159 & $+00: 27: 48.972$ & 0.336 & 0.006 & 0.3149 & 0.0002 & 0.02953 \\
\hline y136 & $2007 t z$ & Ia? & wdd8 & 8 & 02:30:07.153 & $-08: 43: 09.354$ & $\ldots$ & 0.001 & 0.5200 & 0.0010 & 0.03795 \\
\hline y137 & $2007 \mathrm{tt}$ & Ia & wbb8 & 6 & 01:11:20.516 & $-00: 12: 19.423$ & 0.368 & 0.009 & 0.3741 & 0.0004 & 0.02732 \\
\hline y142 & 2007 ud & Ia & wdd 8 & 1 & $02: 30: 13.145$ & $-09: 15: 39.519$ & 0.592 & 0.006 & 0.5820 & 0.0010 & 0.02938 \\
\hline y143 & $2007 u b$ & Ia & wcc2 & 3 & 02:07:24.132 & $-03: 51: 55.226$ & 0.466 & 0.008 & 0.4642 & 0.0003 & 0.02487 \\
\hline y145 & 2007 ua & Ia & wbb3 & 10 & 01:10:31.955 & $+00: 35: 49.434$ & 0.555 & 0.001 & 0.5514 & 0.0003 & 0.02788 \\
\hline y146 & $\ldots$ & Unk & wdd6 & 12 & $02: 29: 26.184$ & $-08: 27: 52.797$ & $\ldots$ & $\ldots$ & $\ldots$ & $\ldots$ & 0.03430 \\
\hline y151 & 2007uc & Ia? & wcc4 & 16 & 02:10:15.529 & $-04: 04: 06.465$ & $\ldots$ & $\ldots$ & 0.5837 & 0.0006 & 0.02255 \\
\hline y154 & $2007 u g$ & Ia? & wec7 & 5 & 02:09:36.845 & $-04: 51: 52.280$ & $\ldots$ & $\ldots$ & 0.6540 & 0.0001 & 0.02246 \\
\hline y155 & $\ldots$ & PISN? & wbb6 & 6 & 01:07:56.085 & $+00: 17: 41.484$ & $\ldots$ & $\ldots$ & 0.7973 & 0.0000 & 0.03096 \\
\hline y156 & 2007ue & Ia & wbb9 & 7 & 01:09:09.843 & $-00: 14: 01.124$ & $\ldots$ & $\ldots$ & 0.6614 & 0.0004 & 0.03364 \\
\hline y158 & 2007uf & Ia & wec5 & 7 & 02:06:30.881 & $-04: 09: 55.047$ & $\ldots$ & $\ldots$ & 0.4856 & 0.0001 & 0.02182 \\
\hline y163 & 2007uh & Ia & wcc3 & 9 & 02:06:05.200 & $-04: 01: 37.458$ & 0.640 & 0.003 & 0.630 & 0.0010 & 0.02411 \\
\hline y173 & $\ldots$ & Ic? & wdd4 & 13 & $02: 35: 34.821$ & $-08: 19: 07.016$ & $\ldots$ & $\ldots$ & 0.4078 & 0.0002 & 0.03593 \\
\hline y175 & 2007ui & Ia & wdd4 & 16 & $02: 34: 57.445$ & $-08: 03: 57.919$ & 0.421 & 0.009 & 0.4130 & 0.0002 & 0.03688 \\
\hline y177 & 2007uj & Ia & wdd4 & 2 & 02:33:19.036 & $-08: 32: 30.217$ & 0.303 & 0.004 & $\ldots$ & $\ldots$ & 0.03212 \\
\hline z179 & $\ldots$ & Gal & wbb8 & 4 & $01: 11: 21.637$ & $-00: 22: 45.114$ & $\ldots$ & $\ldots$ & 0.4462 & 0.0007 & 0.02664 \\
\hline z180 & $2007 \mathrm{uk}$ & Ia & wbb8 & 4 & 01:10:55.006 & $-00: 22: 53.197$ & 0.447 & 0.004 & $\ldots$ & $\ldots$ & 0.02561 \\
\hline z181 & 2007ul & Ia & wec8 & 7 & 02:07:16.534 & $-04: 42: 23.201$ & 0.604 & 0.004 & 0.620 & 0.0010 & 0.02248 \\
\hline z183 & 2007un & Ia & wbb1 & 8 & $01: 14: 22.736$ & $+01: 07: 45.632$ & 0.287 & 0.006 & 0.2806 & 0.0001 & 0.03050 \\
\hline z184 & 2007up & Gal & wcc4 & 3 & 02:09:56.068 & $-04: 28: 57.352$ & $\ldots$ & $\ldots$ & 0.6116 & 0.0002 & 0.02245 \\
\hline z185 & $2007 u v$ & Ia & wbb4 & 9 & 01:15:11.994 & $-00: 02: 08.387$ & 0.414 & 0.005 & 0.4068 & 0.0001 & 0.02924 \\
\hline z187 & $2007 u m$ & Ia & wbb5 & 13 & $01: 12: 29.210$ & $+00: 17: 01.211$ & 0.293 & 0.004 & $\ldots$ & $\ldots$ & 0.03491 \\
\hline z195 & $\ldots$ & Unk & wdd9 & 12 & 02:29:47.674 & $-09: 01: 08.431$ & $\ldots$ & $\ldots$ & $\ldots$ & $\ldots$ & 0.03279 \\
\hline z200 & 2007 uo & Ia & wbb1 & 13 & $01: 14: 43.186$ & $+00: 54: 27.657$ & 0.457 & 0.005 & 0.4548 & 0.0002 & 0.03175 \\
\hline z202 & $2007 u q$ & Ia & wcc3 & 3 & 02:04:21.320 & $-03: 54: 10.960$ & 0.220 & 0.005 & 0.2205 & 0.0002 & 0.02510 \\
\hline z203 & 2007ur & Ia? & wcc2 & 15 & 02:08:41.591 & $-03: 34: 10.527$ & $\ldots$ & 0.001 & 0.2500 & 0.0010 & 0.02317 \\
\hline z204 & 2007us & Ia & wdd3 & 2 & $02: 27: 54.863$ & $-08: 00: 55.479$ & $\ldots$ & $\ldots$ & 0.6201 & 0.0002 & 0.03008 \\
\hline z205 & 2007ut & Ia & wdd8 & 2 & $02: 30: 23.642$ & $-09: 12: 20.022$ & 0.416 & 0.020 & $\ldots$ & $\ldots$ & 0.03042 \\
\hline z208 & 2007 uu & Ia & wbb1 & 5 & 01:14:01.011 & $+00: 53: 47.598$ & 0.502 & 0.008 & 0.5300 & 0.0010 & 0.03017 \\
\hline
\end{tabular}

Notes. 1. Ia-type Ia supernova, no sub-type reported. 2. IaT-similar to the overluminous type Ia supernovae SN $1991 \mathrm{~T}$ or SN 1999 aa. 3 . IaP-similar to peculiar type Ia supernovae SN 2000cx or SN 2002cx. 4. Ib-type Ib supernova, no sub-type reported. 5. Ib-pec-type Ib supernova with peculiar spectral features. 6. Ictype Ic supernova, no sub-type reported. 7. II-type II supernova, no sub-type reported. 8. IIn-type II supernova with narrow emission lines. 9. IIP-type II supernova with a "plateau" in the light curve. 10. II-pec-type II supernova with peculiar spectral features. 11. Classifications followed by a "?" are not definitively spectroscopically typed. 12. PISN? - Possible pair-instability supernova (P. Garnavich 2016, private communication). 13. Gal-Galaxy, sub-types are reported in B. E. Tucker et al. (2014, in preparation). 14. AGN—Active galactic nucleus. 15. Unk-Not observed or could not be classified based on spectra. $z_{\mathrm{SNID}}$ and $z_{\mathrm{GAL}}$ are reported in the heliocentric frame, and must be converted into the cosmic microwave background (CMB) frame, while accounting for local peculiar velocities at low- $z$. For this work, we have employed the Milky Way reddening values from Schlegel et al. (1998), rather than the updated values provided by Schlafly et al. (2012), to facilitate the combination of our objects with literature samples.

offset by $1^{\prime \prime}$. As this is much larger than the typical astrometric uncertainty, we adopt the procedure used by Rest et al. (2014), and find that an offset of 0.3 pixels produces a sub-percent impact on photometry. Since the uncertainty is related to the SNR, we expect increased dispersion at high$z$; however, our cadence provides $\mathrm{S} / \mathrm{N}>10$ for even our highest redshift objects, and we do not find any net bias in the recovered astrometry of known sources with magnitude. We adopted a fixed valued of $0.005 \mathrm{mag}$ in both filters to account for the systematics arising from astrometric uncertainties.

\subsection{Uncertainties in Determining the Airmass Relation}

An error in the slope of the airmass relation would lead to an error in extrapolating the zero points from the Landolt standard fields to the ESSENCE fields, and between the ESSENCE fields. We mitigate this uncertainty by requiring the images we use to extrapolate the zero point for a given image to have a difference in airmass smaller than 0.5 . We introduce a $10 \%$ error in the airmass relation and propagate the error to our photometric catalogs. Such a large error is extremely unlikely, and would be visually apparent, as we obtained images of standard fields over an extended range in airmass, but allows us 
Table 7

Photometry of ESSENCE Objects

\begin{tabular}{|c|c|c|c|}
\hline MJD & Passband & Flux $_{25}$ & $\sigma_{\text {Flux }}$ \\
\hline \multicolumn{4}{|c|}{$\mathrm{k} 425$} \\
\hline 52990.0582 & $R$ & -0.331500 & 0.561100 \\
\hline 52990.0745 & $I$ & -0.013500 & 1.108300 \\
\hline 52994.0601 & $R$ & 0.468100 & 0.468700 \\
\hline 52994.0772 & $I$ & 1.395600 & 0.782300 \\
\hline 53268.1072 & $R$ & 0.029500 & 0.836900 \\
\hline 53268.1484 & $I$ & -0.254100 & 1.248500 \\
\hline 53283.1311 & $R$ & -0.815500 & 0.746300 \\
\hline 53283.1629 & $I$ & 1.064200 & 1.631700 \\
\hline 53289.0561 & $R$ & 0.149500 & 0.764900 \\
\hline 53289.0726 & $I$ & -1.360000 & 1.253400 \\
\hline 53293.0558 & $I$ & 1.089600 & 1.544700 \\
\hline 53297.0640 & $R$ & -0.521300 & 0.616900 \\
\hline 53297.0809 & $I$ & -1.181900 & 0.945800 \\
\hline 53301.0728 & $R$ & 0.085300 & 1.094000 \\
\hline 53301.0973 & $I$ & -1.519200 & 1.085500 \\
\hline 53315.0736 & $R$ & 0.777700 & 0.493500 \\
\hline 53315.0906 & $I$ & 0.207800 & 0.852200 \\
\hline 53323.0829 & $R$ & 10.472000 & 0.477600 \\
\hline 53323.1006 & $I$ & 11.788600 & 0.649700 \\
\hline 53329.0363 & $R$ & 17.830900 & 0.655000 \\
\hline 53329.0533 & $I$ & 24.461000 & 0.892200 \\
\hline 53342.0814 & $R$ & 24.361700 & 0.582500 \\
\hline 53342.0984 & $I$ & 30.781000 & 0.963700 \\
\hline 53346.0734 & $R$ & 20.966300 & 0.543200 \\
\hline 53346.0919 & $I$ & 25.465800 & 1.022600 \\
\hline 53350.0550 & $R$ & 17.523900 & 1.083800 \\
\hline 53350.0724 & $I$ & 21.250500 & 1.189000 \\
\hline 53358.0431 & $R$ & 10.427300 & 0.720000 \\
\hline 53358.0720 & $I$ & 16.345000 & 0.915900 \\
\hline 53360.0755 & $R$ & 10.464500 & 1.257200 \\
\hline 53360.1073 & $I$ & 14.251400 & 2.462600 \\
\hline 53385.0554 & $R$ & 1.924400 & 0.784900 \\
\hline 53385.0589 & $I$ & 4.661300 & 1.151400 \\
\hline 53639.0843 & $R$ & -0.178200 & 0.629700 \\
\hline 53639.1007 & $I$ & 0.131800 & 0.934900 \\
\hline
\end{tabular}

to place an upper limit on the resulting systematic error in magnitudes.

We find that a $\pm 10 \%$ error in the airmass term causes a $\mp 0.001 \mathrm{mag}$ error in $R$ and a $\mp 0.002 \mathrm{mag}$ error in $I$ (see Figure 14). The slightly larger effect in $I$, despite the weaker extinction coefficient, is a result of the smaller number of $I$ images overall, and the larger fraction of $I$ calibration images that were observed at high airmass, relative to $R$.

We also compute the mean difference as a function of magnitude to look for any residual trends. This is critical for SN Ia measurements which span a wide range in magnitude as a function of redshift, light curve shape, and host-galaxy extinction. We find weak $<0.1 \%$ trends as a function of magnitude.

\subsection{Uncertainties in Determining the Photometric Transformation to the Landolt System}

Extinction caused by dust in the host galaxies of the supernovae makes them appear fainter than predicted for their redshift, mimicking the effect of the dark energy. Accurate measurements of SN Ia color are critical in constraining the reddening, and allow us to disentangle the effect of dust from the dark energy signal.
Because high-redshift SN Ia surveys are deep, but cover a small solid angle, they are inefficient at finding large numbers of nearby supernovae. Analysis of the high- $z$ samples requires low $z$ SN Ia from the literature as an anchor for cosmological measurements (Hamuy et al. 1993; Riess et al. 1999; Jha et al. 2006; Hicken et al. 2009b, 2012; Contreras et al. 2010; Ganeshalingam et al. 2010; Stritzinger et al. 2011). Thus, any absolute zero point offsets, even if arising from inaccuracies in the nearby sample, are a common source of systematic error for high-redshift surveys. Because most nearby surveys are tied to the Landolt network, we can estimate a lower bound to this offset by examining how the ESSENCE data are tied to the Landolt system.

In addition, any errors in our $R$ and $I$ flux scaling relative to each other would distort the observed color of the entire sample. At the typical redshifts probed by ESSENCE, our RI photometry covers the rest-frame $B V$, and our inferred host galaxy extinction is related to the measured rest-frame color excess, $E(B-V)$. If we assume that the slope of the reddening law, $R_{V}$, in the host galaxies of our $\mathrm{SN}$ Ia is similar to our own Galaxy $\left(R_{V} \approx 3.1\right)$, then any error in our measured color would lead to an error $\sim 3$ times larger in the extinction, $A_{V}$, and the distance modulus, $\mu$.

SN Ia surveys like ESSENCE are therefore particularly sensitive to systematics affecting measured colors. An error in the photometric transformation can take the form of an error in determining the slope of the color law, or a residual difference in magnitudes around the intercept. The effect of an error in the slope of the color relation is small, as the error is on the order of the product of the error in the color term and the difference between the mean color of our field stars and the color of BD $+17^{\circ} 4708$. We measure the effect of an error in the color term using synthetic photometry, as described in Appendix B.2, and find that $\mathrm{a} \pm 0.02$ error in the slope of the color relation would lead to a $\sim \pm 0.003 \mathrm{mag}$ systematic error in the magnitudes of field stars and derived zero points. We conservatively adopt an error of $0.005 \mathrm{mag}$ as the systematic error resulting from an error in the estimate of the color term.

We measure the residual difference in magnitudes, $m_{4 \mathrm{~m}}$ $m_{\text {Landolt }}$, for our calibration fields around $R-I=0.32 \mathrm{mag}$ in our standard fields and find these to be $-0.003 \mathrm{mag}$ and $-0.001 \mathrm{mag}$ in $R$ and $I$, respectively, with an uncertainty of $\sim 0.001 \mathrm{mag}$ in both bands (see Figure 15). The dispersions about the mean residual are $\sim 1 \%$ in both $R$ and $I$. The residual is consistent with zero in $I$, and of low significance in $R$. We adopt these values as systematic uncertainties in the absolute zero point.

\subsubsection{Comparison to the Sloan Digital Sky Survey}

While we cannot directly compare our magnitudes to Landolt magnitudes in our science fields, we use stars selected from SDSS DR7 and converted onto the Landolt network using transformation equations. This procedure has some limitations: the SDSS imaging is not as deep as MOSAIC II images of the ESSENCE fields, and SDSS magnitudes converted onto the Landolt network have large statistical uncertainties associated with the transformation between two dissimilar photometric systems. In addition, the wcc field is outside the SDSS footprint, and is not included in the analysis. However, as the SDSS photometry was not used in determining our photometric 
Table 8

Quantum Efficiency of the MOSAIC II Imager

\begin{tabular}{|c|c|c|c|c|c|c|c|c|c|}
\hline \multirow{2}{*}{$\begin{array}{l}\text { Wavelength } \\
\text { (§) }\end{array}$} & \multicolumn{9}{|c|}{ Transmission (\%) } \\
\hline & CCD 1 & CCD 2 & CCD 3 & CCD 4 & CCD 5 & CCD 6 & CCD 7 & CCD 8 & Average \\
\hline 3000 & 8.90 & 9.70 & 7.60 & 7.80 & 9.50 & 9.40 & 8.40 & 9.60 & 8.86 \\
\hline 3200 & 18.00 & 18.90 & 15.80 & 16.10 & 18.50 & 18.50 & 18.50 & 19.30 & 17.95 \\
\hline 3340 & 22.70 & 27.90 & 22.40 & 23.00 & 26.40 & 27.10 & 25.40 & 27.60 & 25.31 \\
\hline 3650 & 48.40 & 52.60 & 42.10 & 43.50 & 53.00 & 52.70 & 49.20 & 54.90 & 49.55 \\
\hline 3800 & 62.80 & 56.10 & 56.20 & 57.10 & 62.10 & 61.90 & 58.20 & 65.80 & 60.02 \\
\hline 4050 & 67.50 & 68.80 & 57.90 & 60.90 & 63.90 & 66.90 & 63.40 & 72.10 & 65.17 \\
\hline 4500 & 74.00 & 74.40 & 63.60 & 65.60 & 70.70 & 72.50 & 70.20 & 78.30 & 71.16 \\
\hline 5000 & 77.90 & 79.40 & 69.90 & 73.60 & 76.10 & 77.80 & 75.00 & 81.40 & 76.39 \\
\hline 5500 & 83.20 & 83.90 & 75.30 & 77.70 & 81.60 & 81.40 & 80.30 & 86.50 & 81.24 \\
\hline 6000 & 86.80 & 87.00 & 80.30 & 84.40 & 88.30 & 87.70 & 85.10 & 89.70 & 86.16 \\
\hline 6500 & 87.80 & 88.70 & 82.70 & 86.60 & 89.70 & 89.20 & 87.10 & 90.60 & 87.80 \\
\hline 7000 & 84.70 & 86.20 & 82.80 & 84.50 & 88.40 & 86.30 & 85.70 & 88.10 & 85.84 \\
\hline 7500 & 78.20 & 78.30 & 76.40 & 77.00 & 81.50 & 80.50 & 79.00 & 80.30 & 78.90 \\
\hline 8000 & 68.40 & 68.80 & 67.00 & 68.00 & 71.90 & 68.10 & 69.50 & 70.10 & 68.97 \\
\hline 8500 & 54.00 & 54.50 & 54.70 & 55.30 & 57.60 & 54.00 & 56.50 & 56.10 & 55.34 \\
\hline 9000 & 39.30 & 40.30 & 40.20 & 44.10 & 41.90 & 39.20 & 41.30 & 40.90 & 40.90 \\
\hline 9500 & 24.50 & 25.40 & 25.40 & 25.50 & 26.20 & 24.50 & 26.20 & 26.00 & 25.46 \\
\hline 10000 & 10.90 & 11.60 & 11.80 & 12.20 & 12.00 & 10.60 & 11.80 & 11.70 & 11.57 \\
\hline
\end{tabular}

Table 9

Photon Transmission Function of the ESSENCE Survey

\begin{tabular}{|c|c|c|c|c|c|}
\hline \multirow{2}{*}{$\begin{array}{l}\text { Wavelength } \\
\text { (§) }\end{array}$} & \multicolumn{5}{|c|}{ Transmission (\%) } \\
\hline & $\mathrm{QE}$ & Filter & Optics & Atmosphere & Total \\
\hline \multicolumn{6}{|c|}{$R(\mathrm{c} 6004)$} \\
\hline 5470 & 0.8018 & 0.0000 & 0.9087 & 0.8400 & 0.0000 \\
\hline$\ldots$ & $\ldots$ & $\ldots$ & $\ldots$ & $\ldots$ & $\ldots$ \\
\hline 6240 & 0.8760 & 0.7764 & 0.9021 & 0.8700 & 0.5338 \\
\hline$\ldots$ & $\ldots$ & $\ldots$ & $\ldots$ & $\ldots$ & $\ldots$ \\
\hline 7005 & 0.8546 & 0.4846 & 0.8904 & 0.9160 & 0.3378 \\
\hline$\ldots$ & $\ldots$ & $\ldots$ & $\ldots$ & $\ldots$ & $\ldots$ \\
\hline 7775 & 0.7454 & 0.1784 & 0.8725 & 0.9410 & 0.1092 \\
\hline$\ldots$ & $\ldots$ & $\ldots$ & $\ldots$ & $\ldots$ & $\ldots$ \\
\hline 8540 & 0.5431 & 0.0409 & 0.8719 & 0.9530 & 0.0185 \\
\hline$\ldots$ & $\ldots$ & $\ldots$ & $\ldots$ & $\ldots$ & $\ldots$ \\
\hline 9310 & 0.3111 & 0.0000 & 0.9133 & 0.8530 & 0.0000 \\
\hline \multicolumn{6}{|c|}{$I(\mathrm{c} 6028)$} \\
\hline 6940 & 0.8592 & 0.0000 & 0.8916 & 0.9060 & 0.0000 \\
\hline$\ldots$ & $\ldots$ & $\ldots$ & $\ldots$ & $\ldots$ & $\ldots$ \\
\hline 7635 & 0.7721 & 0.9348 & 0.8763 & 0.5660 & 0.3580 \\
\hline$\ldots$ & $\ldots$ & $\ldots$ & $\ldots$ & $\ldots$ & $\ldots$ \\
\hline 8330 & 0.6052 & 0.9563 & 0.8674 & 0.9420 & 0.4729 \\
\hline$\ldots$ & $\ldots$ & $\ldots$ & $\ldots$ & $\ldots$ & $\ldots$ \\
\hline 9030 & 0.3959 & 0.0070 & 0.9007 & 0.9330 & 0.0023 \\
\hline$\cdots$ & $\ldots$ & $\ldots$ & $\ldots$ & $\ldots$ & $\ldots$ \\
\hline 9725 & 0.1912 & 0.0047 & 0.9275 & 0.9490 & 0.0008 \\
\hline$\cdots$ & $\ldots$ & $\ldots$ & $\ldots$ & $\ldots$ & $\ldots$ \\
\hline 10425 & 0.0000 & 0.0047 & 0.9365 & 0.9690 & 0.0000 \\
\hline
\end{tabular}

calibration, it provides a useful, independent test on our photometric accuracy.

We cross-match stars from Stetson (2005) in SDSS, and extract Übercal (Padmanabhan et al. 2008) corrected magnitudes. We select from SDSS only objects with clean photometry, point-source PSFs, gri uncertainties $<0.1 \mathrm{mag}$ and $\sigma_{z}<0.15 \mathrm{mag}$ without a corresponding entry in the DR7
QSOBest catalog, satisfying

$$
\begin{aligned}
& 0.95<u-g<2.75 \mathrm{mag}, \\
&-0.01<g-r<1.78 \mathrm{mag}, \\
&-0.12<r-i<2.74 \mathrm{mag}, \text { and } \\
&-0.13<i-z<1.58 \mathrm{mag},
\end{aligned}
$$

and close proximity to the stellar locus are selected from SDSS (see Figure 16).

We use simple linear transformations for stars with $r-i<0.8$, determined using the "LINMIX_ERR" routine (Kelly 2007) available in the IDL Astronomy Library. ${ }^{38} \mathrm{We}$ derive the following transformations (see Figure 17) using $>1300$ measured stars:

$$
\begin{aligned}
R_{L} & =r-(0.303 \pm 0.006)(r-i)-(0.133 \pm 0.002) \\
I_{L} & =i-(0.213 \pm 0.007)(r-i)-(0.388 \pm 0.002) .
\end{aligned}
$$

We find large intrinsic dispersions of $\sim 0.025 \mathrm{mag}$ in the relations between the SDSS and Landolt photometry for both $R_{L}$ and $I_{L}$. This dispersion is inherent in the transformation between two photometric systems with very dissimilar transmissions, and significantly different dynamic ranges, and further justifies our choice to base the calibration of the Blanco natural system on the Landolt standard network. We do not find any significant trend in the residuals of transformed $R_{L}$ with $g-r$, or $I_{L}$ with $i-z$. We apply these transformations to SDSS stars in our science fields, selected using the same criteria, to derive their Landolt magnitudes.

We compare our tertiary photometric catalogs for the science fields to SDSS stars, selected using the same criteria as above, converted to Landolt using our derived transformations. No significant field-to-field differences are found. We measure the offset between the CTIO Blanco natural system and these transformed stars around $R-I=0.32 \mathrm{mag}$ as in the standard fields, and found offsets (in the sense of Blanco magnitude minus transformed SDSS magnitude) of $0.009 \pm 0.03$ mag in $R$, and $0.013 \pm 0.03 \mathrm{mag}$ in $I$, consistent with zero. The large

\footnotetext{
38 http://idlastro.gsfc.nasa.gov/
} 

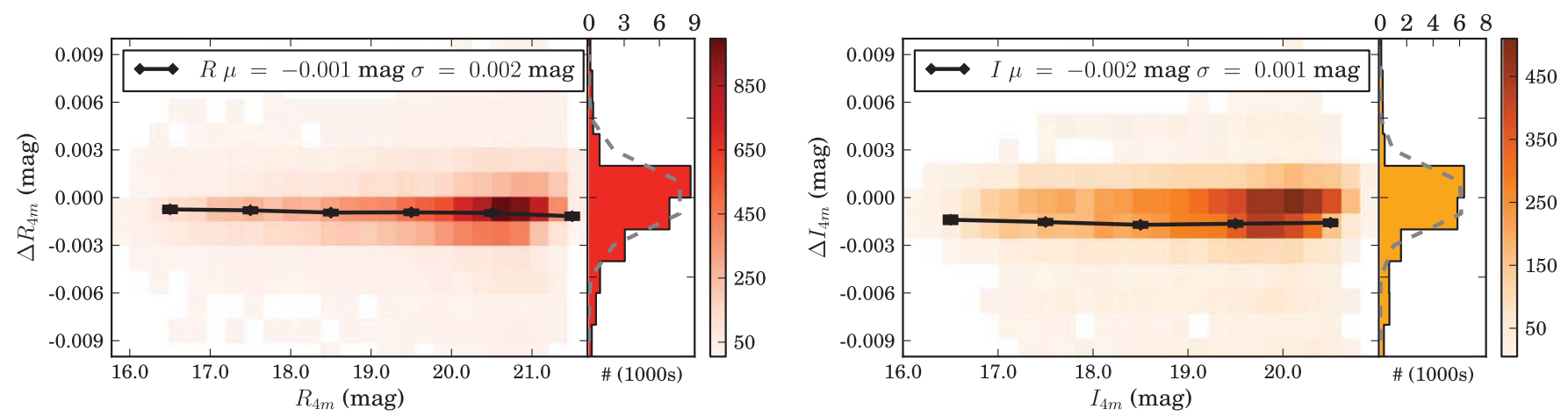

Figure 14. Differences in (left) $R$ - and (right) $I$-band photometry with a $10 \%$ error in the slope of the airmass relation (in the sense of with offset minus no offset), binned as a function of magnitude (left) and for all amplifier images (right). The color bars indicate the number of amplifier images in each bin. The means in bins with 1 mag widths are overplotted (black diamonds). We find a resulting -0.001 mag difference in the $R$ band, and a -0.002 mag difference in the $I$ band. Dispersions are computed using a Gaussian fit to the histogram shown at right.

uncertainties arise from the intrinsic dispersion in the transformation to $R$ and $I$, as well as the $r$ and $i$ uncertainties that are propagated into the uncertainty on the $R-I$.

\subsection{Uncertainties in Extrapolating Photometric Zero Points}

We evaluate the error in determining the photometric zero point for a single amplifier by extrapolating the zero point of the image using the average of all other amplifiers of the same image, and the average of all other images that are within \pm 0.5 in airmass and $\pm 100 \mathrm{~s}$ in exposure time, adjusted for both the difference in airmass and the difference in exposure time. We find the difference between the zero point and the extrapolated zero point to be $<0.001 \mathrm{mag}$. We also construct this statistic field by field and amplifier by amplifier and find no significant difference in these sub-samples. The histogram of differences between the extrapolated and directly fitted zero points are shown in Figure 18.

Additionally, we find that the images with the largest differences between fitted and extrapolated zero points are typically taken in non-photometric conditions and fail quality tests for difference imaging. We find no significant trends in the difference between direct and extrapolated zero points with airmass, aperture correction error, exposure time, FWHM, or sky background. The standard deviations of the un-clipped data are $\sim 0.01 \mathrm{mag}$ in both $R$ and $I$. Either $3 \sigma$ clipping extreme outliers or using a Gaussian to model the data reduces the estimate of the standard deviation to $<0.01 \mathrm{mag}$. This is a strong indication that our internal photometric calibration is no worse than $1 \%$.

\subsection{Uncertainties in Determining the Natural System Magnitudes of $B D+17^{\circ} 4708$}

The CTIO Blanco natural magnitude system adopted in this work utilizes $\mathrm{BD}+17^{\circ} 4708$ as the fundamental spectrophotometric standard and consequently, the magnitudes of $\mathrm{BD}+17^{\circ}$ 4708 in the natural system are close to its Landolt magnitudes by construction. However, there are several astrophysical differences between $\mathrm{BD}+17^{\circ} 4708$ and the "typical" Landolt standard star. We determine the corrections to the first order magnitudes of $\mathrm{BD}+17^{\circ} 4708$ in Appendix B.3. Systematic errors in the magnitudes of $\mathrm{BD}+17^{\circ} 4708$ would lead to an error in the synthetic zero points and $k$-corrections, and the uncertainty budget is dominated by the impact of a potential unresolved binary companion.

\subsection{Uncertainties in the SED of $B D+17^{\circ} 4708$}

While the derivation of the magnitudes of $\mathrm{BD}+17^{\circ} 4708$ in Appendix B relies on the PHOENIX synthetic spectral library (Hauschildt et al. 1997; Sordo et al. 2010, and references therein), the derivation of synthetic zero points requires its true SED. We use the CALSPEC determination of the SED of the $\mathrm{BD}+17^{\circ} 4708$ and adopt a $0.5 \%$ uncertainty over 3000-10000 A. These translate into 0.002 and 0.003 mag differences in the synthetic $R$ and $I$ Blanco magnitudes.

\section{CONCLUSIONS}

We have re-calibrated the CTIO Blanco and MOSAIC II system, with a focus on minimizing the systematic errors that originate from photometry and affect the high-redshift SN Ia measurements from ESSENCE. This calibration supersedes that presented by Miknaitis et al. (2007), and improves on it by deriving photometric transformations between the Landolt network and the Blanco natural system without employing any observations from the CTIO $0.9 \mathrm{~m}$, avoiding cross-telescope systematics.

Additionally, we have selected $\mathrm{BD}+17^{\circ} 4708$ as the fundamental spectrophotometric standard in this work. The $R-I$ color of this standard is considerably closer to the average color of Landolt network stars, as well as SN Ia around the median redshift of ESSENCE. This choice minimizes systematic errors arising from errors in determining the photometric transformation between the Landolt and Blanco natural systems.

We employed these transformations to derive secondary photometric catalogs for our Landolt calibration fields that span the MOSAIC II field of view. We demonstrated that we could accurately extrapolate zero points between the different amplifiers of the imager. Tertiary catalogs were derived for ESSENCE fields, and zero points were established for our imaging. The zero points in both passbands are stable relative to each other over the entire duration of the survey. We have provided a model of the system response of the $R$ and $I$ passbands used by ESSENCE, and made a comprehensive estimate of the effect of various systematics on magnitudes in both passbands.

The primary application of this work is the calibration of light curves of SN Ia discovered by ESSENCE to derive the equation of state parameter of the dark energy, $w$. We have outlined our spectroscopic follow up, and classification program to identify SN Ia within survey data, and presented 

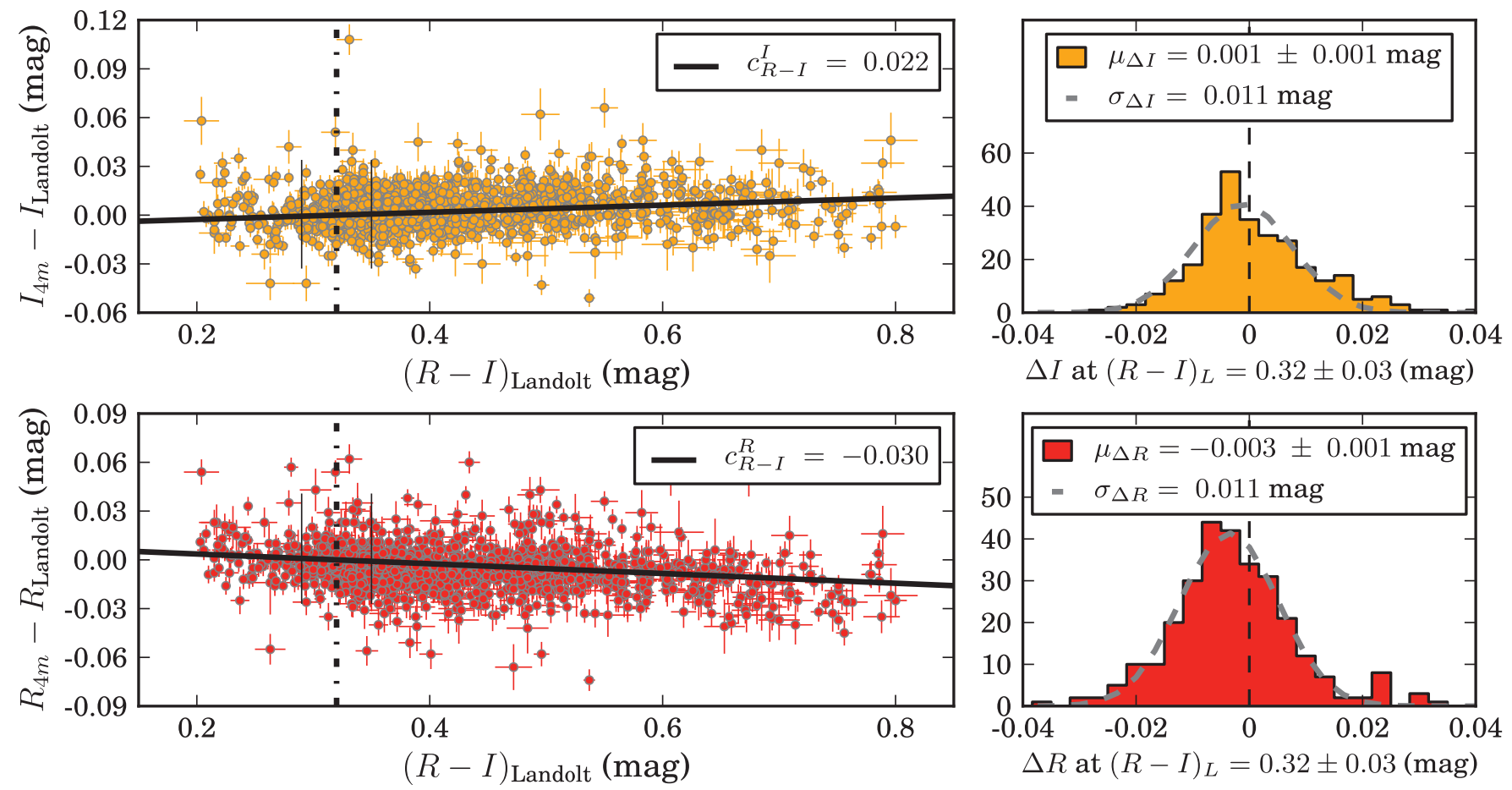

Figure 15. Differences in the (upper left) $I$ - and (lower left) $R$-band magnitude between the CTIO Blanco and the Landolt network in the standard fields. The solid line indicates the slope of the color relation. The slopes are determined at an intercept of $R-I=0.32$ mag. We examine the residuals in a range of \pm 0.03 mag (this range is indicated by short vertical lines in the left panels) around the color intercept. We find the differences in both $R$ (lower right) and $I$ (upper right) are consistent with zero, indicating that there is no significant residual offset after the color transformation is determined.

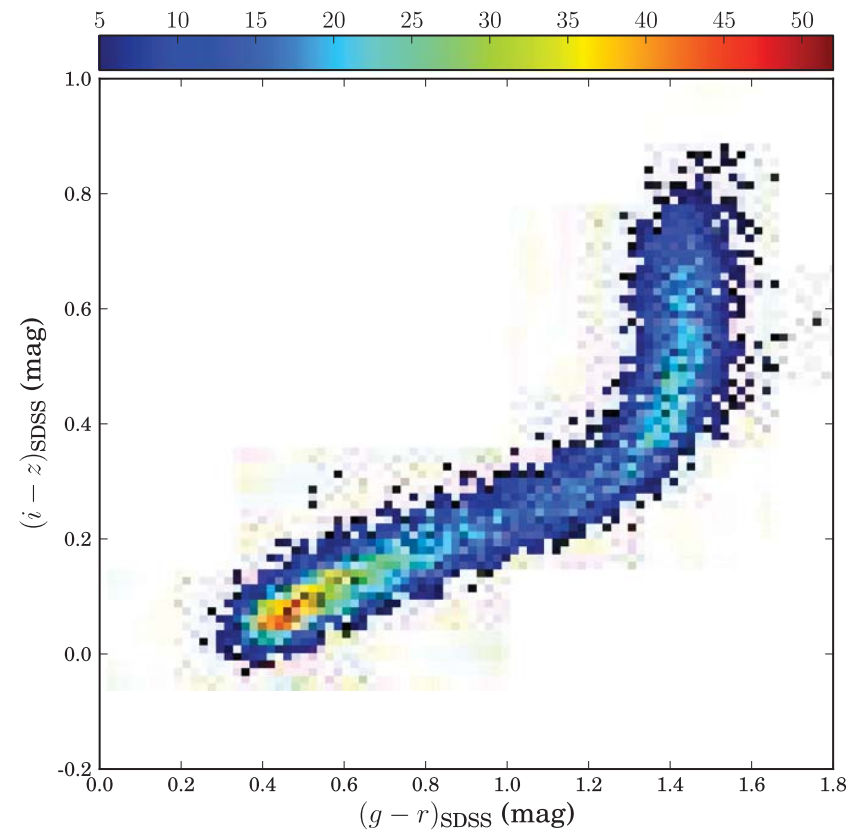

Figure 16. Color-color diagram of SDSS stars in ESSENCE fields. Only stars with $r-i<0.8$ are used to derive transformations to Landolt and assess our absolute photometric consistency.

calibrated light curves in the CTIO Blanco natural system of 213 SN Ia discovered by ESSENCE.

There remain several potential areas for improvement where the calibration presented in this paper may be further refined. Our imaging of Landolt standard fields was obtained during our 2006-2007 observing seasons. Consequently, we only use science images obtained on the same nights to derive our tertiary catalogs, rather than all survey images. While there are clear changes in the zero points over the course of the survey, the lack of standard field imaging covering the same range of time prevents us from deriving the absolute CTIO-to-Landolt photometric transformation as a function of time. As our fundamental spectrophotometric standard, $\mathrm{BD}+17^{\circ} 4708$, was not directly observed using the CTIO Blanco, we derived estimates of its natural system magnitudes using the CTIO-toLandolt transformations, together with Landolt photometry and the PHOENIX synthetic spectral library, to characterize the effect of metallicity, surface gravity, and extinction. However, the principal shortcoming of the ESSENCE SN Ia photometry remains its lack of multi-color information. This increases our sensitivity to priors on the colors or extinction of SN Ia. Nevertheless, this work demonstrates that the systematic errors from photometry are $\sim 1 \%$ in both $R$ and $I$. This represents a better understanding of the systematic errors arising from photometric calibration, and an overall reduction of its impact on the ESSENCE systematic error budget.

In future work, we will combine our calibrated light curves with our spectroscopic observations as well as SN Ia host galaxy information (B. E. Tucker et al. 2014, in preparation) to derive accurate distance moduli from ESSENCE. We will combine our measurements with those from other low and high-redshift SN Ia surveys to place constraints on cosmological parameters.

G.N. is supported by NSF award AST-0507475 and the Department of Energy. Based in part on observations obtained at the Cerro Tololo Inter-American Observatory (CTIO), part of the National Optical Astronomy Observatory (NOAO), 

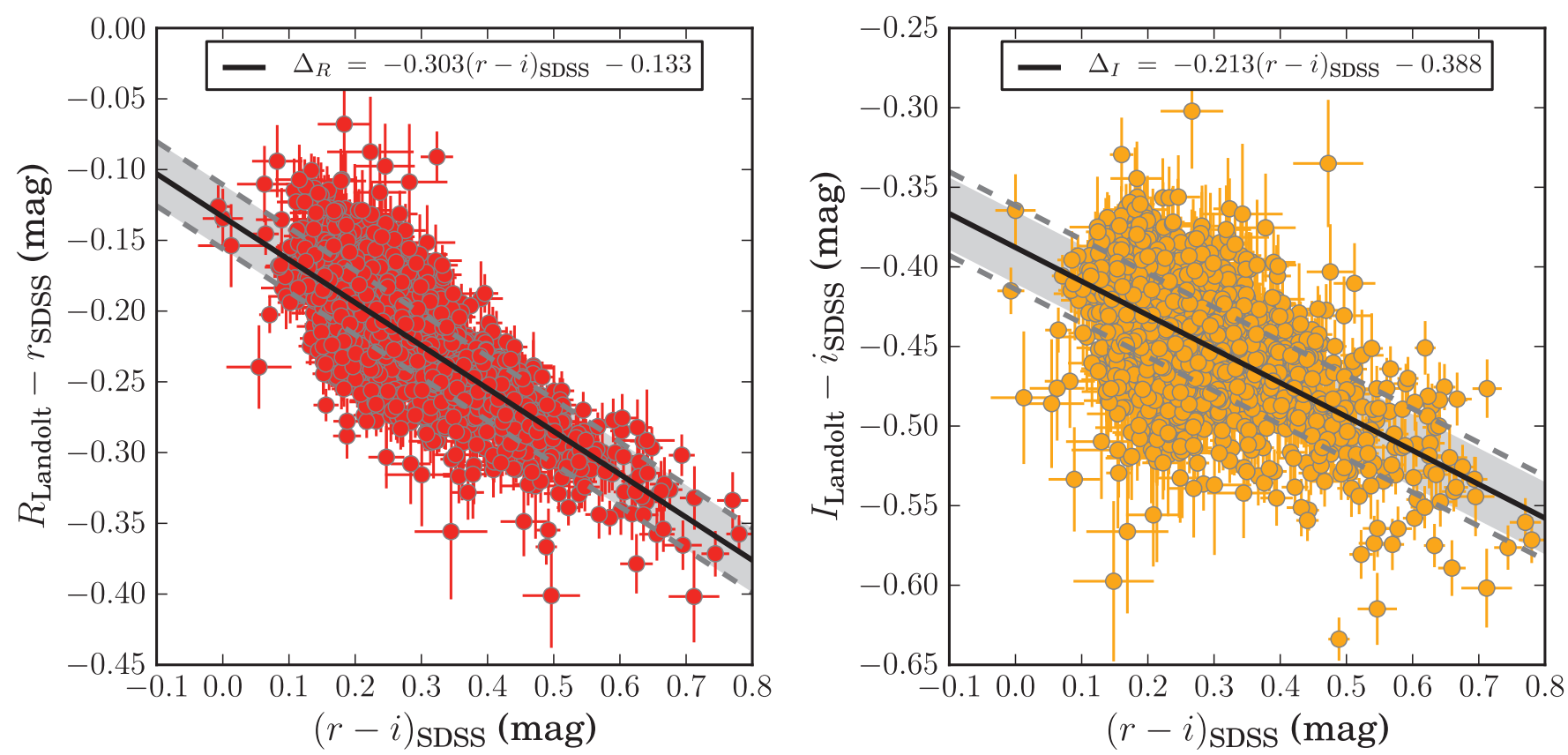

Figure 17. Transformations between the Landolt and SDSS photometric system using stars observed by Stetson for $R$ (left) and $I$ (right). The shaded gray region enclosed between dashed gray lines indicates the intrinsic dispersion in the fit and is $\sim 0.025$ mag for both transformations.

which is operated by the Association of Universities for Research in Astronomy, Inc. (AURA) under cooperative agreement with the National Science Foundation (NSF); the European Southern Observatory, Chile (ESO Programmes 170. A-0519 and 176.A-0319); the Gemini Observatory, which is operated by the Association of Universities for Research in Astronomy, Inc., under a cooperative agreement with the NSF on behalf of the Gemini partnership: the NSF (United States), the Particle Physics and Astronomy Research Council (United Kingdom), the National Research Council (Canada), CONICYT (Chile), the Australian Research Council (Australia), CNPq (Brazil) and CONICET (Argentina) (Programs GN2002B-Q-14, GS-2003B-Q-11, GN-2003B-Q-14, GS-2004BQ-4, GN-2004B-Q-6, GS-2005B-Q-31, GN-2005B-Q-35); the Magellan Telescopes at Las Campanas Observatory; the MMT Observatory, a joint facility of the Smithsonian Institution and the University of Arizona; and the F.L. Whipple Observatory, which is operated by the Smithsonian Astrophysical Observatory. Some of the data presented herein were obtained at the W. M. Keck Observatory, which is operated as a scientific partnership among the California Institute of Technology, the University of California, and the National Aeronautics and Space Administration. The Observatory was made possible by the generous financial support of the W. M. Keck Foundation.

A.V.F.'s group at UC Berkeley received additional assistance from NSF grants AST-0908886 and AST-1211916, the TABASGO foundation, and the Christopher R. Redlich fund. The Dark Cosmology Centre is funded by the Danish National Research Foundation. The ESSENCE survey team is very grateful to the scientific and technical staff at the observatories we have been privileged to use.

The survey is supported by the U.S. National Science Foundation through grants AST-0443378 and AST-0507475. The Dark Cosmology Centre is funded by the Danish National Research Foundation.
Our project was made possible by the survey program administered by NOAO, and builds upon the data reduction pipeline developed by the SuperMACHO collaboration. IRAF is distributed by the National Optical Astronomy Observatory, which is operated by AURA under cooperative agreement with the NSF.

We made extensive use of the Odyssey Cluster administered by the FAS-IT Research Computing Group at Harvard, and are very grateful to the staff there.

J.M.S. is supported by an NSF Astronomy and Astrophysics Postdoctoral Fellowship under award AST-1302771. A.C. acknowledges support from grant IC120009 awarded to the Millennium Institute of Astrophysics, MAS, by the Ministry of Economy, Development and Tourism, and grant Basal CATA PFB 06/09 from CONICYT..

Facilities: Blanco (MOSAIC II), CTIO:0.9 m (CFCCD), Gemini:South (GMOS), Gemini:Gillett (GMOS), Keck:I (LRIS), Keck:II (DEIMOS, ESI), VLT (FORS1), Magellan: Baade (IMACS), Magellan:Clay (LDSS2).

\section{APPENDIX A \\ ESTIMATION AND PROPERTIES OF THE ILLUMINATION CORRECTION}

Flat-field images obtained with the CTIO Blanco are corrected using an illumination correction derived from science images as described in Section 3.1. Here we detail the estimation of the illumination corrections and their time dependence, and quantify the associated systematic errors.

\section{A.1. Deriving the Illumination Correction}

We create our illumination corrections using the following prescription.

1. Create a master dome flat from the set of dome flats, $F_{D}$.

2. Calibrate science frames, $F_{S}$, with the master dome flats.

3. Mask out all stars in the resulting science frames. 

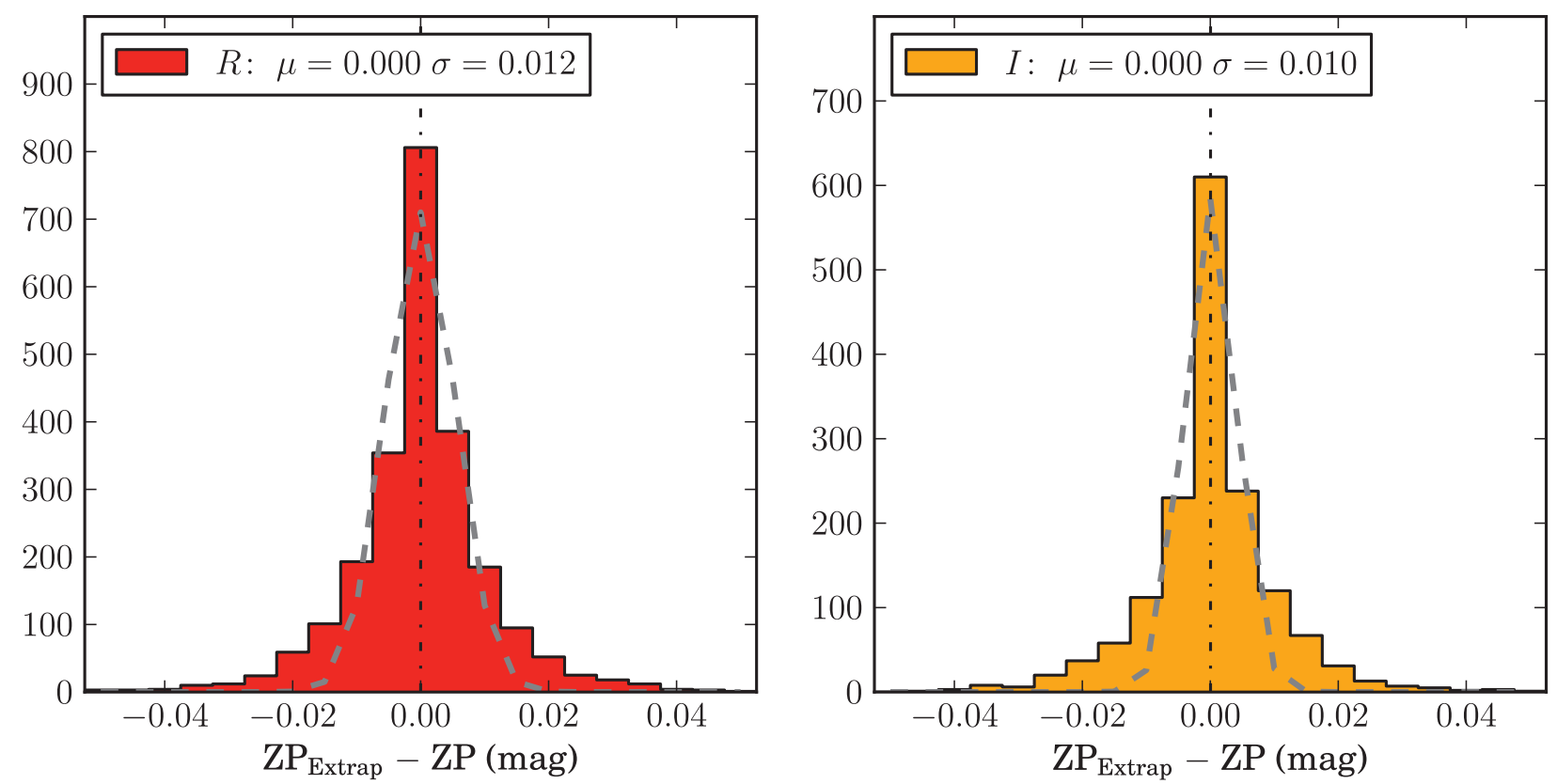

Figure 18. Differences between the fitted zero point of an amplifier image and the zero point extrapolated from the average of the other amplifiers of the same image, in $R$ (left) and $I$ (right). We find no net offset between the directly fitted and extrapolated zero points. In addition, the standard deviation of the residuals normalized by the uncertainties is close to 1 , indicating that the uncertainties are well modeled.

4. Normalize the masked science frames to the same average sky value.

5. Average the resulting frames to produce one combined image.

6. Normalize the combined image to a mean of unity, and take the multiplicative inverse.

7. Smooth the normalized combined image with a large kernel to the generate final illumination correction.

8. Multiply the dome flat with the illumination correction.

Mathematically, we can describe our final illumination correction, $I(t)$, as:

$$
I=S_{K}\left(\left\langle\frac{F_{S}}{\left\langle F_{D}\right\rangle}\right\rangle^{-1}\right),
$$

where $S_{K}$ represents the smoothing kernel used in the stage, and angled braces denote the average. We bin each $1024 \times 4096$ pixels amplifier image by a factor of 4 , and smooth the binned image with $30 \times 30$ pixels, before re-expanding the binned image to the original dimensions. This effective 120 pixel scale is larger than the small scale structures of the flat field, such as out-of-focus dust "donuts," while retaining the large-scale gradients that we seek to correct for. Finally, we construct a master illumination-corrected flat-field image, $F_{I}$, via

$$
F_{I}(t)=F_{D}(t) \times I(t),
$$

where $I$ and $F_{D}$ are normalized to an average value of 1.0, and we introduce $t$ denotes the night of observation. The illumination-corrected flat-field image is used to flatten the science images from the night.

\section{A.2. Temporal Stability of the Illumination Correction}

We distinguish two types of changes affecting the optical system.
1. Global changes that affect all images, including new dust grains on the optics, changes in instrument mounting, and mechanical changes in the mirror support.

2. Flat field changes that only affect our dome flat images, including ghosting, non-uniformity of the flat-field screen, as well as instances where a flat-field lamp burnt out.

We examine the temporal stability of the illuminationcorrected flat fields when subjected to both types of changes. From Equations (8) and (9), provided the global changes are small, the product $F_{I}(t)$ should not be sensitive to changes in the dome flats. We determine the ratios of dome flat, illumination correction, and illumination-corrected flat fields for all nights within an observing run. This is illustrated in Figure 19, where we compare frames between 20030927 and 20031020. The ratio of the illumination-corrected flat-field images is within $0.1 \%$ of unity, despite differences at the $0.5 \%$ level between the flat field and illumination correction frames. We calculate the standard deviation of the ratio images, as well as the maximum difference between the ratio image and the average of the ratio. Nights for which the standard deviation of the ratio is consistently $>0.1 \%$, or the maximum error of the ratio is consistently $>0.3 \%$, are flagged. Comparing our flagged nights to subjective observing logs for the nights, we find that flagged nights have excessive moonlight. This difference presumably arises from non-focusing light paths producing stray light illumination of the focal plane, with an intensity pattern different from the light path for focused celestial sources. We find that the illumination corrections degrade more rapidly toward full moon in $R$ than $I$, and attribute this to the steeper gradients in the sky brightness in $R$. This temporal stability is exploited to estimate an illumination correction for flagged nights using other nights within the observing run. A $0.3 \%$ error is adopted as the systematic on the illuminationcorrection frames. 


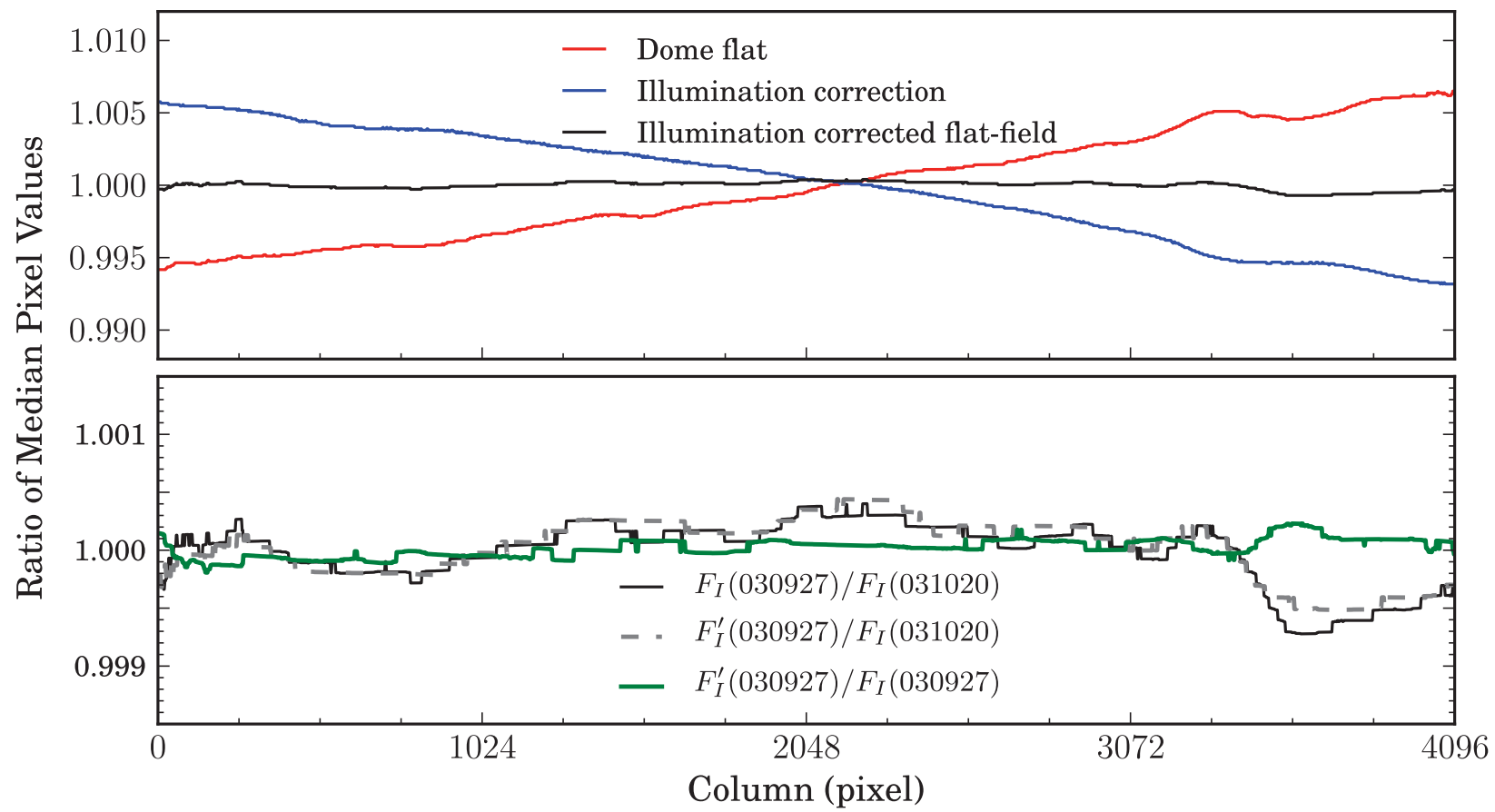

Figure 19. Illustration of the stability of the illumination corrections from 20030927 (randomly selected) and 20031020 ( $\sim 1$ month later). Top: The ratio of the dome flat frames is shown in red, while the ratio of the illumination correction frames is shown in blue. Both ratios indicate that there are differences at the $\sim 0.5 \%$ level between these two nights. The ratio of the illumination-corrected flat field between the two nights, shown in black, is within $0.1 \%$ of unity, indicating that the illumination correction is accurately accounting for the variations in the dome flat images, despite being separated by almost a month. Bottom: The ratio of the illumination-corrected flat fields between 20030927 and 20031020 is again shown in black on a finer scale to illustrate the structure. We construct an estimated illumination correction for 20030927, $F_{I}^{\prime}$, using the flat and bias images from 20031020, and the science frames from 20030927. The ratio of the flat-field image processed with the estimated illumination correction and the illumination-corrected flat field for 20031020 is shown as a dashed gray line. The ratio of the derived and the estimated illumination-corrected flat fields on 20030927 is shown in green, and illustrates that the illumination-corrected flat fields are stable to better than $0.1 \%$ between the two dates.

\section{APPENDIX B \\ PROPERTIES OF THE CTIO 4 M NATURAL SYSTEM}

We describe the properties of the CTIO Blanco natural system in the following subsections. We derive the system transmission and compute synthetic color terms to the Landolt system. We use our determined transmission along with synthetic photometry of model SEDs to study the differences between our fundamental spectrophotometric standard, BD $+17^{\circ} 4708$, and "typical" Landolt stars at similar colors. Finally, we establish synthetic zero points to derive natural system magnitudes from flux-calibrated SEDs.

\section{B.1. Transmission}

We model the transmission, $T$, of the CTIO Blanco system by the product of four components: the atmosphere (Atm), optics (Opt), filter (PB), and the Quantum Efficiency of the MOSAIC II CCDs (QE).

$$
T(\lambda)=T_{\mathrm{Atm}}(\lambda) \times T_{\mathrm{Opt}}(\lambda) \times T_{\mathrm{PB}}(\lambda) \times \mathrm{QE}(\lambda) .
$$

\section{B.1.1. Detector Quantum Efficiency}

The eight Tek CCDs that comprise the MOSAIC II have slightly different quantum efficiencies (listed in Table 8). However, we find the differences in synthetic photometry from using different quantum efficiency curves is $<0.001 \mathrm{mag}$, for both $R$ and $I$ over a wide range of color. Consequently, we elect to use a single average value of the quantum efficiency for all the CCDs.

\section{B.1.2. $R$ and I Optical Filters}

The MOSAIC II uses filters that are $146 \times 146 \mathrm{~mm}$ and $\sim 12 \mathrm{~mm}$ thick. The transmissions of the $R$ (NOAO code c6004) and $I$ filter (c6028) were measured by CTIO staff ${ }^{39}$ using an OceanOptics S2000 spectrometer. The S2000 is a crossed Czerny-Turner spectrometer, configured with a $600 \mathrm{ln} / \mathrm{mm}$ grating, blazed at $750 \mathrm{~nm}$, for measurements over 600-1200 nm. Measurements were obtained through a $10 \mu \mathrm{m}$ wide slit coupled to a fiber optic with $400 \mu \mathrm{m}$ core diameter. The resulting optical resolution is $\sim 10 \mathrm{~nm}$ FWHM. The filters are illuminated with a General Electric 787 halogen lamp with quartz bulb, identical to those used to illuminate the Blanco flat-field screen, through a ground glass diffuser. The spectrum is projected onto a $1 \times 2048$ pixel CCD array and digitized. An OceanOptics HG-1 He-Ar lamp produces reference spectral features to determine the pixel-to-wavelength transformation. The transformation is modeled as a simple cubic polynomial. The central wavelength of the filters is shifted $\sim 15 \AA$ to the blue when mounted in the prime focus of the $f / 2.87$ beam with ADC, relative to measurements at normal incidence. The shift is included in the provided transmission curve.

\footnotetext{
$\overline{39}$ http://www.ctio.noao.edu/noao/content/mosaic-filters
} 


\section{B.1.3. Telescope Optics}

As the MOSAIC II is mounted at prime focus, the transmission of the optics is dominated by the wavelengthdependent reflectivity of the primary mirror, and is well modeled by the reflectivity of aluminium. The transmission of the $\mathrm{ADC}^{40}$ was measured to above $85 \%$ in the range 3500 $8500 \AA$. The transmission of the ADC does fall significantly in the ultraviolet, but this has no effect on our RI photometry. The drop off at the red end is very gradual and the transmission at $10000 \AA$ is $\sim 75 \%$.

\section{B.1.4. Atmospheric Transmission}

M07 used a model of the atmospheric transmission derived from observations of spectrophotometric standards, with the removal of telluric features. The resulting atmospheric model, while reasonably precise, depends on the standard used, and the details of the reduction, particularly on the fit of a smooth psuedocontinuum. We generate an atmospheric model using the MODTRAN4 code. The generated atmosphere is appropriate for an airmass of 1, and consists of $2 \mathrm{~mm}$ PMW of water vapor at an altitude of $2 \mathrm{~km}$, convolved with the atmospheric scattering function and the transmission from aerosols. The differences between our atmospheric model and that employed by M07 are primarily in the strength of the absorption features, with the largest differences on the red wing of the $I$ band $(>9500 \AA)$. The differences result in an $<0.001 \mathrm{mag}$ change in synthetic colors over a wide range (note that the M07 transmission is provided in erg $/ \AA$ and must be divided by $\lambda$ for comparison with this work).

The total system throughput at an airmass of unity is listed in Table 9. Measurements of the system throughput using a tunable laser, calibrated to a NIST photodiode, were consistent with the product of each component (Stubbs et al. 2007). We could not measure the system throughput of the $I$ filter (c6005) used very early in the survey and replaced after significant damage in 2002 November.

\section{B.2. Synthetic Color Relations}

We derive synthetic color terms between the CTIO natural system and the Landolt network using a procedure similar to that of Stritzinger et al. (2005). We approximate the Landolt passbands using the Cousins $R_{C}$ and $I_{C}$ transmissions published in Bessell (1990), convolved with a model atmosphere, and shifted in wavelength by a small amount $\Delta \lambda$. The shifts are determined by comparing the observed Landolt photometry of the non-variable standards in the spectral library of Stritzinger et al. (2005) to their synthetic photometry, and shifting the passbands, without shifting the atmospheric features, until the $R$ and $I$ synthetic and observed photometry agreed, with a color term consistent with zero in $V-R, V-I$, and $R-I$. We find that the $R$ and $I$ Bessell filters have to be blueshifted by $36 \AA$ and $12 \AA$ in $R$ and $I$, respectively. Using our determination of the CTIO system throughput in Table 9, we compare synthetic photometry of the spectral library to synthetic photometry through the shifted Bessell passbands. We derive synthetic Landolt-to-CTIO color transformations, finding $c_{R-I}^{R}(\mathrm{Syn})=-0.033$ and $c_{R-I}^{I}(\mathrm{Syn})=0.047$. The results of this analysis are presented in Figure 20.

\footnotetext{
$\overline{40}$ http://www.ctio.noao.edu/mosaic/manual/pfadc_paper.ps
}

The synthetic color term in $R$ is in excellent agreement with the color term determined from photometric observations, but there is a significant discrepancy in $I$. A blueshift of $\sim 40 \AA$ to the $I$ Bessell transmission is required to reproduce the observed Landolt-to-CTIO color term in $I$, but a shift of this size introduces a non-zero $R-I$ color term between the observed and synthetic Landolt magnitudes. There is no wavelength shift for the Bessell determination of $I$, such that the synthetic and observed Landolt magnitudes and the synthetic CTIO and synthetic Landolt magnitudes simultaneously agree with nonzero color terms. Fundamentally, approximating the Landolt $I$ passband by a shifted Bessell $I$ filter is not accurate, as the shapes of these filters differ. Specifically, the transmission in the $I$ band is significantly affected by the roll-off in the detector quantum efficiency, which is not included in the Bessell determination. The detector quantum efficiency is effectively constant over $R$, and therefore has an insignificant effect on the shape of the transmission. Current and future surveys observing in griz will be able to calibrate to photometric systems such as SDSS, Pan-STARRS, and SkyMapper, which have wellmeasured system responses.

\section{B.3. The Magnitudes of $B D+17^{\circ} 4708$ in the CTIO Blanco Natural System}

The transformations defined by Equation (4) are constructed such that, to first order, the natural system magnitudes of BD $+17^{\circ} 4708$ are equal to its Landolt magnitudes in $R$ and $I$. However, since we could not observe $\mathrm{BD}+17^{\circ} 4708$ directly, we determine the coefficients of the transformation equations using the Landolt network of stars. In this subsection, we quantify the difference in natural system magnitudes between $\mathrm{BD}+17^{\circ} 4708$, and Landolt stars having $R-I$ color similar to it by:

$$
\delta L_{T}=M_{4 \mathrm{~m}}-M_{L}-c_{(R-I)_{L}}^{M_{4 \mathrm{~m}}}\left((R-I)_{L}-0.32\right) .
$$

By construction, the average residual $\langle\delta L\rangle=0$ for average Landolt stars. Following Regnault et al. (2009), we consider the photometric residual arising from metallicty and surface gravity, extinction differences between $\mathrm{BD}+17^{\circ} 4708$ and the average Landolt star, and consider the systematic effect of a possible faint, unresolved companion. The various effects considered are illustrated in Figure 21.

\section{B.3.1. Metallicity and Surface Gravity}

We determine the difference in synthetic $R$ and $I 4 \mathrm{~m}$ magnitudes residuals between $\mathrm{BD}+17^{\circ} 4708$ and "typical" Landolt stars with metallicity, $[\mathrm{M} / \mathrm{H}]=-0.5$ and $\log$ $(g)=4.0$ as a function of the difference in synthetic $R-I$ color, over $5600 \mathrm{~K}<T_{\text {eff }}<6500 \mathrm{~K}$. We find the relationship between the mean magnitude residual, and difference in $R-I$ color to be linear for both $R$ and $I$. We determine the intercept at $R-I=0.32$, and find (in the sense of $\mathrm{BD}+17^{\circ} 4708 \mathrm{mag}$ minus Landolt mag) that $\delta L<0.001$ mag for both $R$ and $I$.

To measure the effect of surface gravity alone, we select synthetic SEDs with the same parameters as above, except at $\log (g)=4.5$. We measure the difference in the residual to normal Landolt stars, $\delta L_{M}$, caused by perturbing the synthetic SEDs from $\log (g)=4.0$ to $\log (g)=4.5$. We find the effect of changing surface gravity on the difference in residual (in the sense of residual at $\log (g)=4.5$ minus residual at $\log$ $(g)=4.0)$ is $\delta L_{R}<0.001 \mathrm{mag}$, while $\delta L_{I} \approx+0.002 \mathrm{mag}$. 

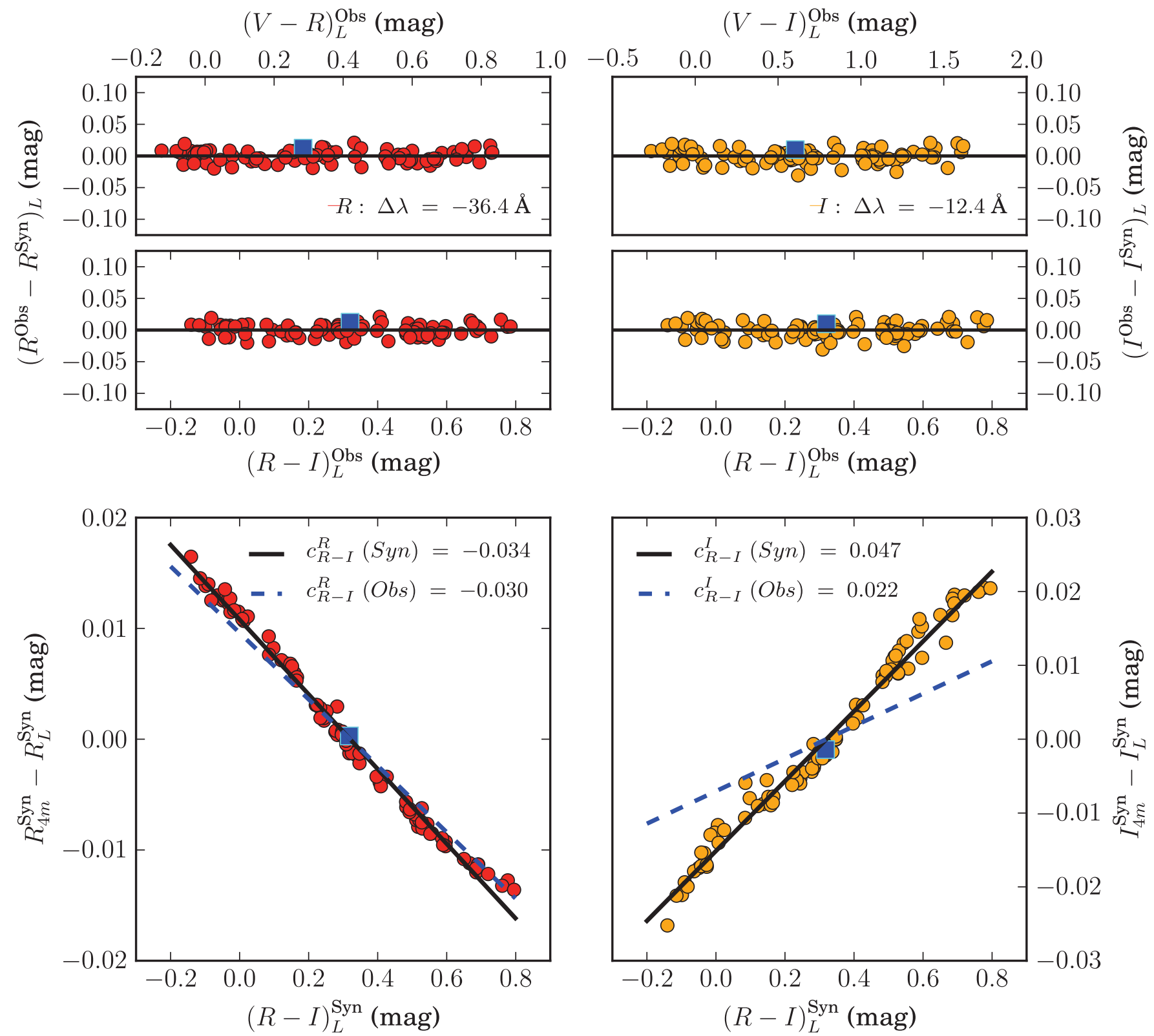

Figure 20. Top and middle: Residuals between observed (Obs) Landolt magnitudes and synthetic (Syn) magnitudes of 99 non-variable stars in the spectral library of Stritzinger et al. (2005) as a function of the observed Landolt color indicated for $R$ (left panels) and $I$ (right panels). The Landolt passbands are modeled by shifting the Bessell (1990) determinations in wavelength by $\Delta \lambda=-36 \AA$ and $-12 \AA$ in $R$ and $I$, respectively. A solid black line at $\Delta M=0$ is included as a visual guide. Bottom: Synthetic color transformations between our determination of the CTIO system throughput and the model Landolt system throughput for $R$ (left) and $I$ (right), respectively. The observed color relations from photometric measurements is indicated by dashed blue lines, while the best-fit relation to the synthetic photometry is indicated by a solid black line. There is excellent agreement in $R$. We believe that the disagreement in $I$ is a result of not modeling the roll-off in the detector quantum efficiency for the model Landolt throughput. A blueshift of $-40 \AA$ is sufficient to recover the observed Landolt-to-CTIO color term in $I$, but introduces a small color term between the observed and synthetic Landolt measurements in $R-I$. The observed and synthetic photometry of BD $+17^{\circ} 4708$, using the CALSPEC SED, is indicated by a blue square in all the plots. There is a $\sim 1 \%$ offset between the flux calibration of the CALSPEC BD $+17^{\circ} 4708$ SED, and the mean flux calibration of the Stritzinger et al. (2005) spectral library.

The combined effect of metallicity and surface gravity leads to a negligible difference in $R$ and a net $\delta L_{I}$ of $\sim 0.001 \mathrm{mag}$.

\section{B.3.2. Extinction}

Regnault et al. (2009) express the distance of $\mathrm{BD}+17^{\circ} 4708$ from the stellar locus in $V-R, R-I$ in terms of the effect of the difference in extinction and the difference in metallicity (the effect of surface gravity being negligible over the color range in question). Having determined the effect of a difference in metallicity using a procedure similar to that above, they found the difference in the reddening between $\mathrm{BD}+17^{\circ} 4708$ and Landolt stars of similar color to be $\Delta E(B-V) \sim 0.045$ mag.

We redden the synthetic SED of $\mathrm{BD}+17^{\circ} 4708$ by this amount, and examine the difference in the residual to normal Landolt stars (in the sense of residual with reddened SED minus residual with un-reddened SED) to be less than 0.001 mag in $R$ and $\approx 0.001$ mag in $I$. 

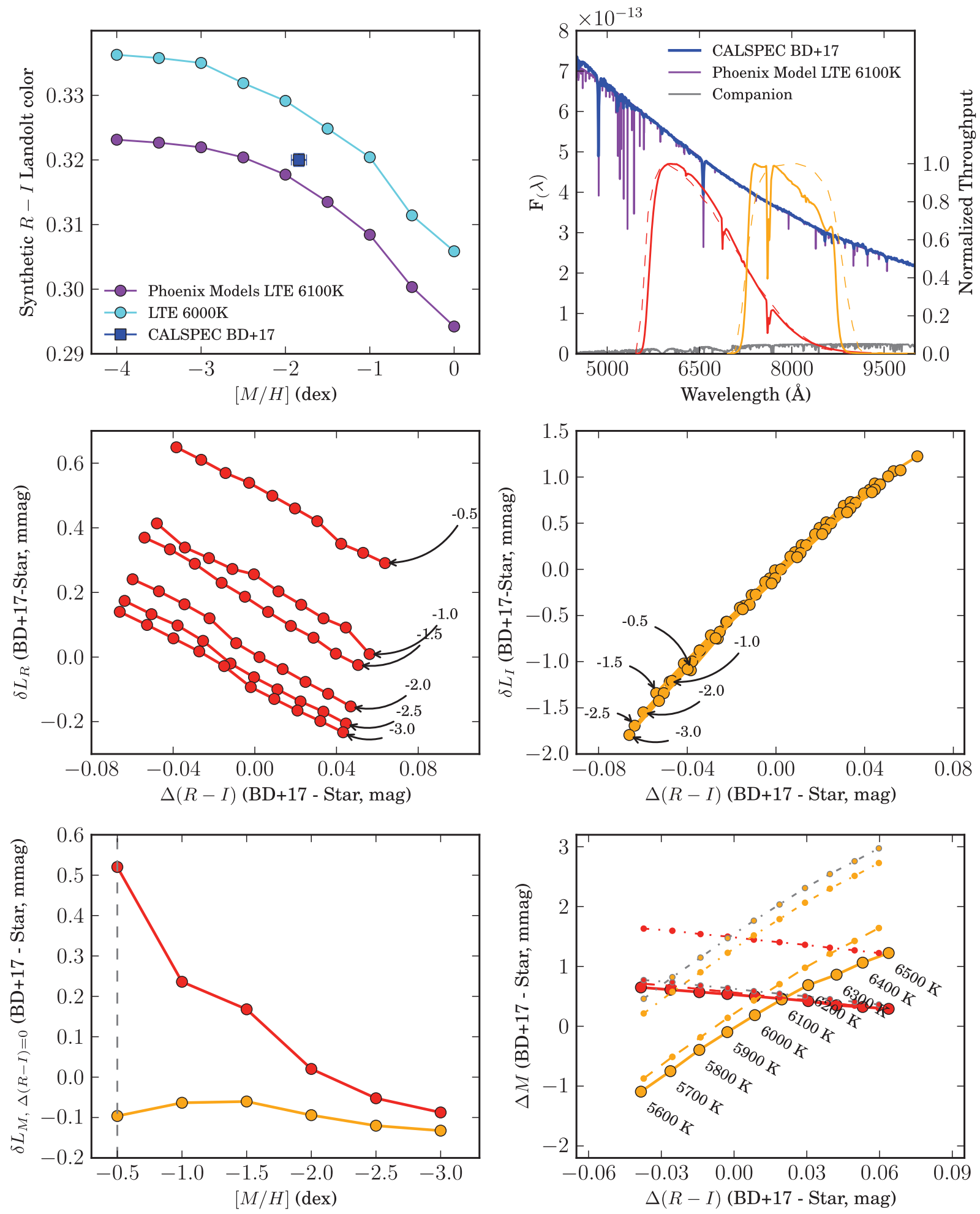

Figure 21. Synthetic magnitudes of BD $+17^{\circ} 4708$ in the CTIO Blanco Natural System. Top left: Synthetic colors of Phoenix SEDs as a function of metallicity, compared to the synthetic color of the CALSPEC SED of BD $+17^{\circ} 4708$. The best model has $T_{\mathrm{eff}}=6100 \mathrm{~K}$ and $[\mathrm{M} / \mathrm{H}]=-2.0 \mathrm{dex}$. Top right: Comparison of the CALSPEC SED and the adopted Phoenix model. The adopted model for the companion of BD $+17^{\circ} 4708$ has $T_{\text {eff }}=3000 \mathrm{~K}$ and $[\mathrm{M} / \mathrm{H}]=-2.0 \mathrm{dex}$. Normalized CTIO Blanco (solid) and Bessell (dashed) transmissions in $R$ (red) and $I$ (orange) are shown for comparison. Middle: Difference in photometric residual, $\delta L$, (in the sense of $\mathrm{BD}+17$ mag minus Landolt mag $\left.\left(T_{\mathrm{eff}},[\mathrm{M} / \mathrm{H}]\right)\right)$ over a range of temperature and metallicity for $R$ (mid left) and $I(\mathrm{mid}$ right) vs. the difference in $R-I$ color (in the sense of $\mathrm{BD}+17$ color minus Landolt color). The effect of changing metallicity is negligible in $I$. Bottom left: The values of $\delta L$ at $\Delta(R-I)=0$ for the different metallicities. The typical metallicity of Landolt stars $([\mathrm{M} / \mathrm{H}]=-0.5 \mathrm{dex})$ is indicated by the vertical line. Bottom right: Deviations from the relation of $\delta L$ at $[\mathrm{M} / \mathrm{H}]=-0.5$ dex are shown for changes in surface gravity (dashed), extinction (dot-dashed), and the addition of a companion (dot-dashed gray) for $R$ (red) and $I$ (orange). 
The combined effect of the difference in metallicity, surface gravity, and extinction is found to be $\delta L_{R}=0.001 \mathrm{mag}$ and $\delta L_{I}=0.002 \mathrm{mag}$. These offsets are added to the first order estimates of the magnitudes of $\mathrm{BD}+17^{\circ} 4708$.

\section{B.3.3. Binarity}

Using the estimates from Ramírez et al. (2006) for the companion of $\mathrm{BD}+17^{\circ} 4708\left(T_{\mathrm{eff}}=3000 \mathrm{k}, \log (\mathrm{g})=4.5\right.$, and $[\mathrm{M} / \mathrm{H}]=-2)$, we compute the difference in photometric residuals and find (in the sense of with companion minus without companion) than $\delta L_{R, I} \approx 0.001 \mathrm{mag}$. As we do not know the fraction of Landolt stars that are also in binaries, we treat these offsets as systematic errors.

\section{B.4. Photometric Zero Points}

With the Landolt magnitudes of $\mathrm{BD}+17^{\circ} 4708 \quad(R=$ $9.166 \mathrm{mag}$ and $I=8.846 \mathrm{mag}$ ) and the photometric residuals caused by the differences in metallicity, surface gravity, and extinction to typical Landolt stars computed in the previous subsection, we invert Equation (3) to derive synthetic passband zero points for $R$ and $I$ and find:

$$
\begin{aligned}
\mathrm{ZP}_{R_{4 \mathrm{~m}}} & =-21.649 \pm 0.001 \mathrm{mag} \\
\mathrm{ZP}_{I_{4 \mathrm{~m}}} & =-22.305 \pm 0.002 \mathrm{mag} .
\end{aligned}
$$

These values differ from the values determined using the Stritzinger et al. (2005) SED library by $0.012 \mathrm{mag}$. This discrepancy is likely the result of a difference in flux calibration between CALSPEC and Stritzinger et al. (2005), as illustrated in Figure 20. We use the CALSPEC SED of BD $+17^{\circ} 4708$ for the determination of passband zero points, as its flux calibration is not affected by atmospheric transmission, and has been carefully studied by several groups.

\section{B.5. Differences in Natural System Definition to the Four-year Data Release}

M07 tied the natural system of the Blanco to Landolt using $\alpha$ Lyr as their fundamental standard, with $(R-I)_{\text {Landolt }}=0$. In addition, a slightly steeper $c_{R-I}^{I}$ color term was employed in that work, and we expect a difference on the order of the product of difference of the color terms, and the average color of Landolt stars, $\left\langle(R-I)_{\text {Landolt }}\right\rangle$.

To first order, the differences between the photometry of stars in this work and M07 are the result of the differences between the definition of the photometric system in Equation (4) and the M07 definition:

$$
\begin{aligned}
\Delta R \approx & c_{R-I}^{R} \times(R-I)_{\mathrm{BD}+17} \\
\approx & -0.030 \times 0.32 \\
\approx & -0.01 \mathrm{mag} \\
\Delta I \approx & c_{R-I}^{I} \times(R-I)_{\mathrm{BD}+17} \\
& +\Delta c_{R-I}^{I} \times\left(\left\langle(R-I)_{\text {Landolt }}\right\rangle-(R-I)_{\mathrm{BD}+17)}\right) \\
\approx & (0.030 \times 0.32)-0.008 \times(0.47-0.32) \\
\approx & 0.009 \mathrm{mag} .
\end{aligned}
$$

However, we have taken various measures to improve the calibration of the natural system, with a view to minimizing our overall photometric error budget, as discussed is Section 3. Consequently, the methodology used in this paper differs substantially from that used by M07. In particular, this work uses observations of the ESSENCE fields tied directly to Landolt fields, whereas M07 tied the Blanco photometry to $0.9 \mathrm{~m}$ observations of field stars, that were in turn tied to Landolt. This is a potential source of additional differences above the expected $1 \%$ level.

\section{REFERENCES}

Abazajian, K. N., Adelman-McCarthy, J. K., Agüeros, M. A., et al. 2009 , ApJS, 182, 543

Albert, J., Brown, Y., Stubbs, C., et al. 2014, in American Astronomical Society Meeting Abstracts 224, 40501

Albrecht, A., Bernstein, G., Cahn, R., et al. 2006, arXiv:astro-ph/0609591

Allington-Smith, J., Breare, M., Ellis, R., et al. 1994, PASP, 106, 983

Appenzeller, I., Fricke, K., Fürtig, W., et al. 1998, The ESO Messenger, 94, 1

Astier, P., Guy, J., Regnault, N., et al. 2006, A\&A, 447, 31

Barone-Nugent, R. L., Lidman, C., Wyithe, J. S. B., et al. 2012, MNRAS, 425, 1007

Barris, B. J., Tonry, J. L., Novicki, M. C., \& Wood-Vasey, W. M. 2005, AJ, 130, 2272

Bertin, E., Mellier, Y., Radovich, M., et al. 2002, in ASP Conf. Ser. 281, Astronomical Data Analysis Software and Systems XI, ed. D. A. Bohlender, D. Durand, \& T. H. Handley (San Francisco, CA: ASP), 228

Bessell, M. S. 1990, PASP, 102, 1181

Betoule, M., Kessler, R., Guy, J., et al. 2014, A\&A, 568, A22

Betoule, M., Marriner, J., Regnault, N., et al. 2013, A\&A, 552, A124

Blondin, S., \& Tonry, J. L. 2007, ApJ, 666, 1024

Blondin, S., Mandel, K. S., \& Kirshner, R. P. 2011, A\&A, 526, A81

Blondin, S., Walsh, J. R., Leibundgut, B., \& Sainton, G. 2005, A\&A, 431, 757

Bohlin, R. C. 2014, AJ, 147, 127

Bohlin, R. C., Gordon, K. D., \& Tremblay, P.-E. 2014, PASP, 126, 711

Conley, A., Guy, J., Sullivan, M., et al. 2011, ApJS, 192, 1

Conley, A., Sullivan, M., Hsiao, E. Y., et al. 2008, ApJ, 681, 482

Contreras, C., Hamuy, M., Phillips, M. M., et al. 2010, AJ, 139, 519

Davis, T. M., Mörtsell, E., Sollerman, J., et al. 2007, ApJ, 666, 716

Dressler, A. 2004, A User's Manual for IMACS

Faber, S. M., Phillips, A. C., Kibrick, R. I., et al. 2003, Proc. SPIE, 4841, 1657

Fernie, J. D. 1981, PASP, 93, 333

Filippenko, A. V. 1997, ARA\&A, 35, 309

Fischler, M. A., \& Bolles, R. C. 1981, Commun. ACM, 24, 381

Folatelli, G., Phillips, M. M., Burns, C. R., et al. 2010, AJ, 139, 120

Foley, R. J., \& Kasen, D. 2011, ApJ, 729, 55

Foley, R. J., Filippenko, A. V., Kessler, R., et al. 2012, AJ, 143, 113

Foley, R. J., Matheson, T., Blondin, S., et al. 2009, AJ, 137, 3731

Freedman, W. L., Burns, C. R., Phillips, M. M., et al. 2009, ApJ, 704, 1036

Fukugita, M., Ichikawa, T., Gunn, J. E., et al. 1996, AJ, 111, 1748

Ganeshalingam, M., Li, W., Filippenko, A. V., et al. 2010, ApJS, 190, 418

Garg, A., Stubbs, C. W., Challis, P., et al. 2007, AJ, 133, 403

Goldhaber, G., Groom, D. E., Kim, A., et al. 2001, ApJ, 558, 359

Guy, J., Astier, P., Baumont, S., et al. 2007, A\&A, 466, 11

Guy, J., Sullivan, M., Conley, A., et al. 2010, A\&A, 523, A7

Hamuy, M., Maza, J., Phillips, M. M., et al. 1993, AJ, 106, 2392

Hamuy, M., Phillips, M. M., Suntzeff, N. B., et al. 1996, AJ, 112, 2391

Hauschildt, P. H., Baron, E., \& Allard, F. 1997, ApJ, 483, 390

Hayes, D. S., \& Latham, D. W. 1975, ApJ, 197, 593

Hicken, M., Challis, P., Jha, S., et al. 2009b, ApJ, 700, 331

Hicken, M., Challis, P., Kirshner, R. P., et al. 2012, ApJS, 200, 12

Hicken, M., Wood-Vasey, W. M., Blondin, S., et al. 2009a, ApJ, 700, 1097

Hook, I., Allington-Smith, J. R., Beard, S. M., et al. 2003, Proc. SPIE, 4841, 1645

Horne, K. 1986, PASP, 98, 609

Ivezić, Ž., Smith, J. A., Miknaitis, G., et al. 2007, AJ, 134, 973

Jha, S., Kirshner, R. P., Challis, P., et al. 2006, AJ, 131, 527

Jha, S., Riess, A. G., \& Kirshner, R. P. 2007, ApJ, 659, 122

Johnson, H. L., \& Morgan, W. W. 1953, ApJ, 117, 313

Kelly, B. C. 2007, ApJ, 665, 1489

Kelly, P. L., Hicken, M., Burke, D. L., Mandel, K. S., \& Kirshner, R. P. 2010, ApJ, 715, 743

Kessler, R., Becker, A. C., Cinabro, D., et al. 2009, ApJS, 185, 32

Krisciunas, K., Bastola, D., Espinoza, J., et al. 2013, AJ, 145, 11

Krisciunas, K., Hastings, N. C., Loomis, K., et al. 2000, ApJ, 539, 658 
Krisciunas, K., Phillips, M. M., \& Suntzeff, N. B. 2004, ApJL, 602, L81 Lampeitl, H., Smith, M., Nichol, R. C., et al. 2010, ApJ, 722, 566

Landolt, A. U. 1983, AJ, 88, 439

Landolt, A. U. 1992, AJ, 104, 340

Landolt, A. U., \& Uomoto, A. K. 2007, AJ, 133, 768

Maguire, K., Sullivan, M., Ellis, R. S., et al. 2012, MNRAS, 426, 2359

Mandel, K. S., Narayan, G., \& Kirshner, R. P. 2011, ApJ, 731, 120

Mandel, K. S., Wood-Vasey, W. M., Friedman, A. S., \& Kirshner, R. P. 2009, ApJ, 704, 629

Matheson, T., Blondin, S., Foley, R. J., et al. 2005, AJ, 129, 2352

Miknaitis, G., Pignata, G., Rest, A., et al. 2007, ApJ, 666, 674

Nordin, J., Östman, L., Goobar, A., et al. 2011, ApJ, 734, 42

O’Donnell, J. E. 1994, ApJ, 422, 158

Oke, J. B., Cohen, J. G., Carr, M., et al. 1995, PASP, 107, 375

Oke, J. B., \& Schild, R. E. 1970, ApJ, 161, 1015

Padmanabhan, N., Schlegel, D. J., Finkbeiner, D. P., et al. 2008, ApJ, 674,1217

Perlmutter, S., Aldering, G., Goldhaber, G., et al. 1999, ApJ, 517, 565

Peterson, D. M., Hummel, C. A., Pauls, T. A., et al. 2006, Natur, 440, 896

Phillips, M. M. 1993, ApJL, 413, L105

Phillips, M. M., Lira, P., Suntzeff, N. B., et al. 1999, AJ, 118, 1766

Prieto, J. L., Rest, A., \& Suntzeff, N. B. 2006, ApJ, 647, 501

Ramírez, I., Allende Prieto, C., Redfield, S., \& Lambert, D. L. 2006, A\&A, 459,613

Regnault, N., Conley, A., Guy, J., et al. 2009, A\&A, 506, 999

Rest, A., Scolnic, D., Foley, R. J., et al. 2014, ApJ, 795, 44

Rest, A., Stubbs, C., Becker, A. C., et al. 2005, ApJ, 634, 1103

Riess, A. G., Filippenko, A. V., Challis, P., et al. 1998, AJ, 116, 1009

Riess, A. G., Kirshner, R. P., Schmidt, B. P., et al. 1999, AJ, 117, 707

Riess, A. G., Press, W. H., \& Kirshner, R. P. 1996, ApJ, 473, 88

Riess, A. G., Strolger, L.-G., Casertano, S., et al. 2007, ApJ, 659, 98

Sako, M., Bassett, B., Becker, A. C., et al. 2014, arXiv:1401.3317

Schechter, P. L., Mateo, M., \& Saha, A. 1993, PASP, 105, 1342
Schlafly, E. F., Finkbeiner, D. P., Jurić, M., et al. 2012, ApJ, 756, 158 Schlegel, D. J., Finkbeiner, D. P., \& Davis, M. 1998, ApJ, 500, 525

Schmidt, G. D., Weymann, R. J., \& Foltz, C. B. 1989, PASP, 101, 713

Scolnic, D., Rest, A., Riess, A., et al. 2014b, ApJ, 795, 45

Scolnic, D. M., Riess, A. G., Foley, R. J., et al. 2014a, ApJ, 780, 37

Sheinis, A. I., Bolte, M., Epps, H. W., et al. 2002, PASP, 114, 851

Silverman, J. M., Ganeshalingam, M., Li, W., \& Filippenko, A. V. 2012, MNRAS, 425, 1889

Sordo, R., Vallenari, A., Tantalo, R., et al. 2010, Ap\&SS, 328, 331

Stetson, P. B. 2000, PASP, 112, 925

Stetson, P. B. 2005, PASP, 117, 563

Stritzinger, M. D., Phillips, M. M., Boldt, L. N., et al. 2011, AJ, 142, 156

Stritzinger, M., Suntzeff, N. B., Hamuy, M., et al. 2005, PASP, 117,810

Stubbs, C. W., Doherty, P., Cramer, C., et al. 2010, ApJS, 191, 376

Stubbs, C. W., Slater, S. K., Brown, Y. J., et al. 2007, in ASP Conf. Ser. 364, The Future of Photometric, Spectrophotometric and Polarimetric Standardization, ed. C. Sterken (San Francisco, CA: ASP), 373

Stubbs, C. W., \& Tonry, J. L. 2006, ApJ, 646, 1436

Sullivan, M., Conley, A., Howell, D. A., et al. 2010, MNRAS, 406, 782

Taylor, B. J. 1986, ApJS, 60, 577

Tonry, J., \& Davis, M. 1979, AJ, 84, 1511

Tonry, J. L., Stubbs, C. W., Lykke, K. R., et al. 2012, ApJ, 750, 99

Tucker, D. L., Annis, J. T., Lin, H., et al. 2007, in ASP Conf. Ser. 364, The Future of Photometric, Spectrophotometric and Polarimetric Standardization, ed. C. Sterken (San Francisco, CA: ASP), 187

Walker, E. S., Hook, I. M., Sullivan, M., et al. 2011, MNRAS, 410, 1262

Wang, X., Filippenko, A. V., Ganeshalingam, M., et al. 2009, ApJL, 699, L139

Wood-Vasey, W. M., Friedman, A. S., Bloom, J. S., et al. 2008, ApJ, 689,377

Wood-Vasey, W. M., Miknaitis, G., Stubbs, C. W., et al. 2007, ApJ, 666, 694

Zacharias, N., Urban, S. E., Zacharias, M. I., et al. 2004, AJ, 127, 3043 